\title{
Distributed Multimodal Interaction Protocol: Enabling Transport of Distributed Interactions
}

\author{
Lucas Stephenson
}

A thesis submitted to the Faculty of Graduate and Postdoctoral Affairs in partial fulfillment of the requirements for the degree of

Master of Applied Science

in

Human-Computer Interaction

Carleton University

Ottawa, Ontario

(C) 2013

Lucas Stephenson 


\begin{abstract}
A growing number of areas in the world have ubiquitous access to mobile, personal technology provided through a variety of hardware form factors; smartphones, tablets, and more. Despite this, the ability to use these personal devices to control and interact with remote technology is limited further progress is mired by proprietary technologies that places this form of remote access technology in isolated software silos. This project provides an open standard networking protocol to enable transport of multimodal interactions between disparate endpoints, with minimal reliance on the personal devices' software or hardware platform. This allows technology enablers to more easily design, develop, deploy and maintain flexible software solutions. Simplifying the software development life cycle allows for more access to technology by end users and can increase the resources available for user experience considerations. The defined protocol is validated through a number of sample implementation tests and by verifying its ability to transport multimodal interaction information.
\end{abstract}




\section{Acknowledgements}

I would like to express my very great appreciation to Dr. Anthony Whitehead for his valuable and constructive suggestions during the planning and development of this research work. His willingness to give his time so generously has been very much appreciated.

I would also like to thank the staff and students of the Carleton $\mathrm{HCl}$ program for their moral and academic support.

Additionally I would like to thank Susan and Meaghan Pinard for their continued support.

Finally, I wish to thank my family for their support and encouragement throughout my completion of this work. 


\section{Table of Contents}

Abstract ii

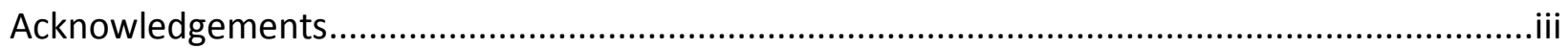

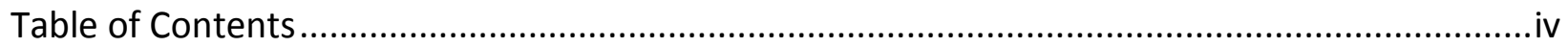

List of Tablesvii

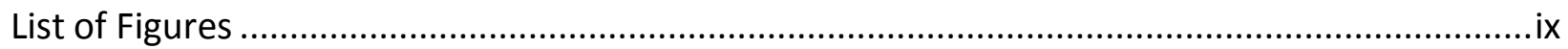

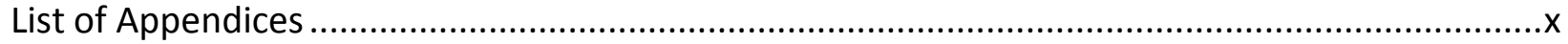

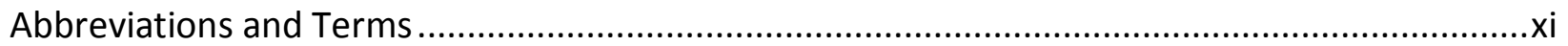

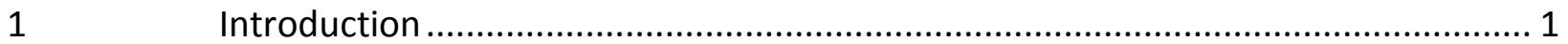

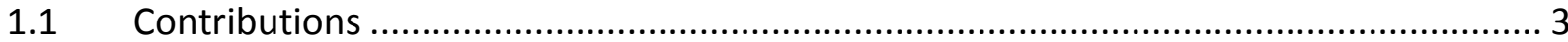

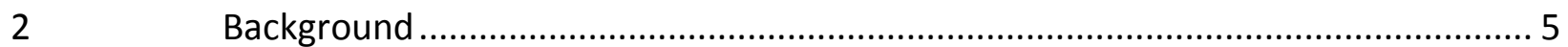

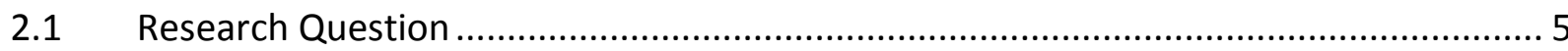

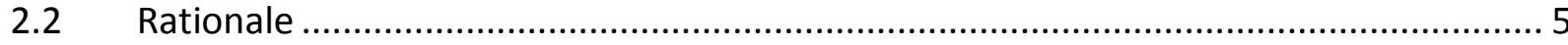

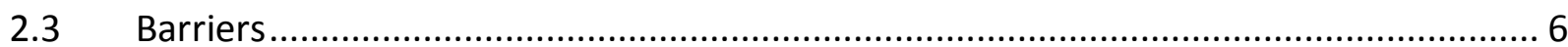

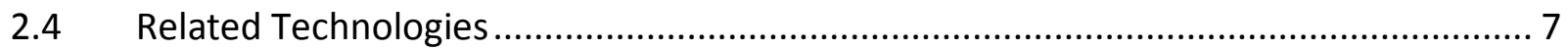

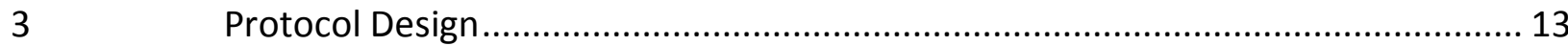

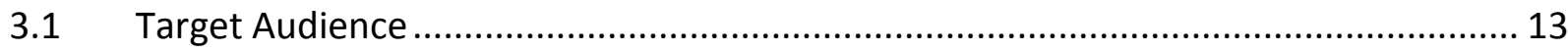

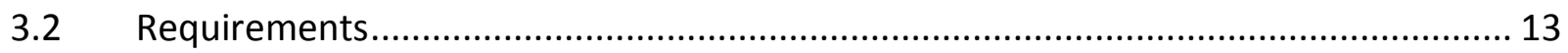

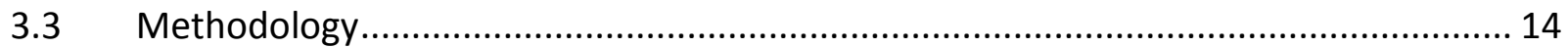

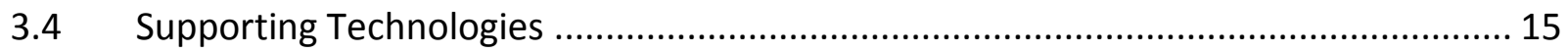

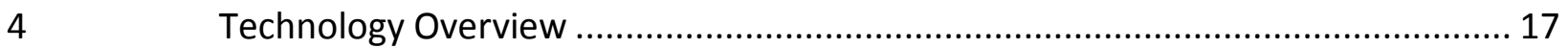

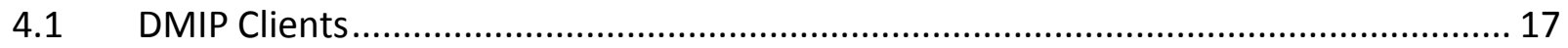

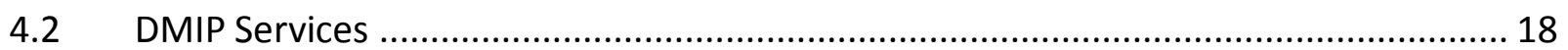

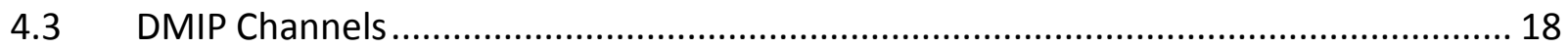

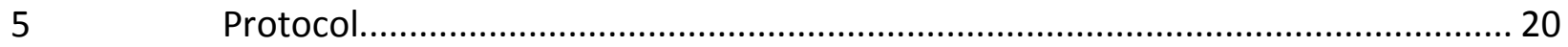

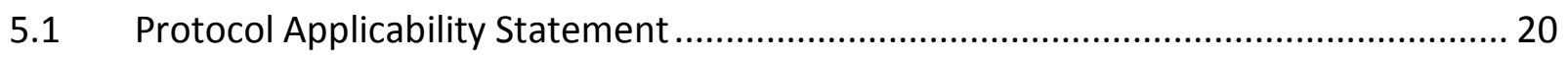

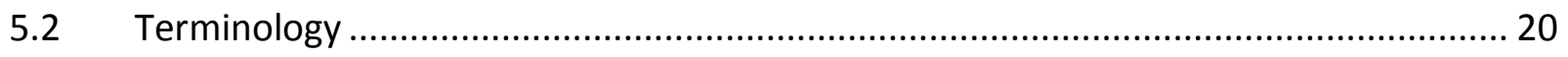

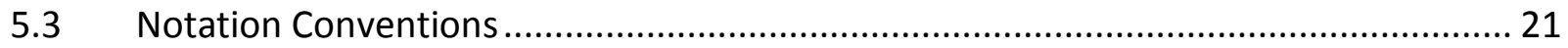

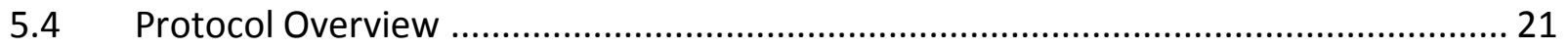

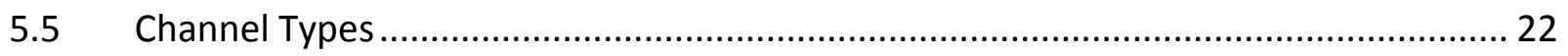

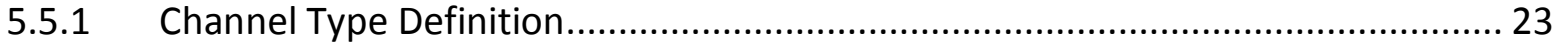




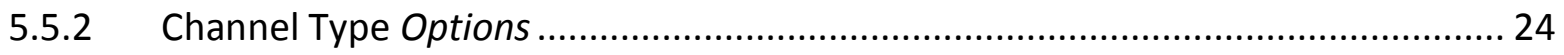

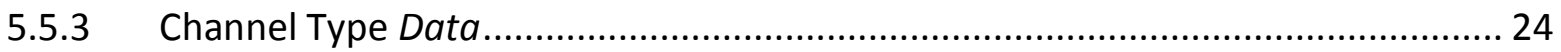

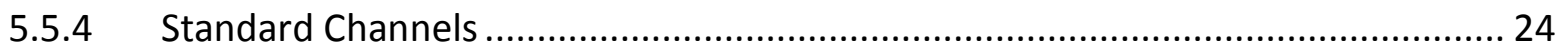

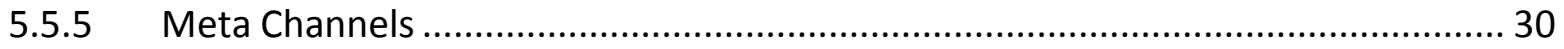

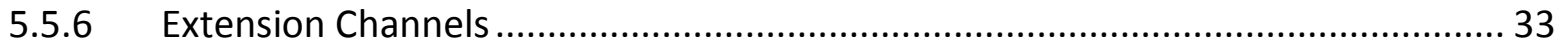

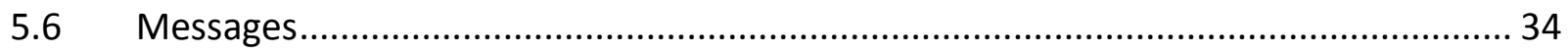

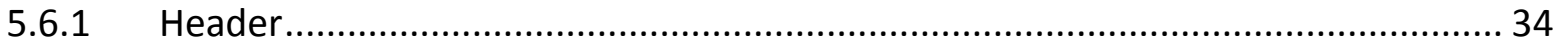

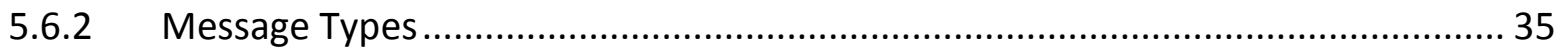

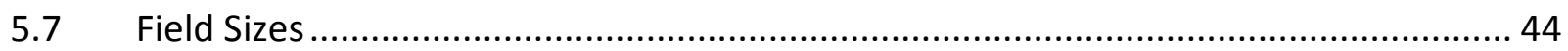

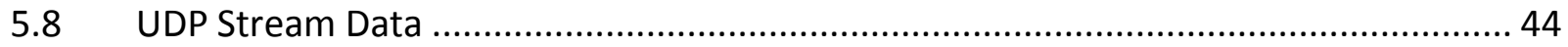

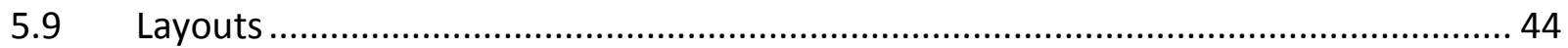

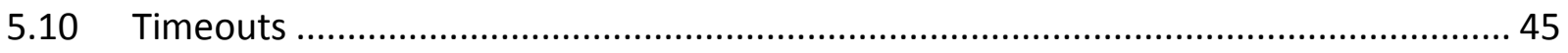

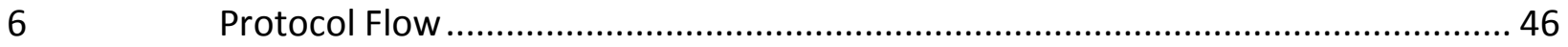

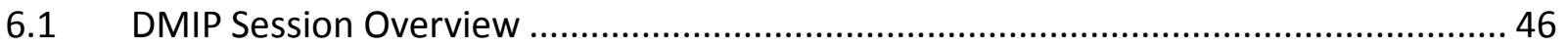

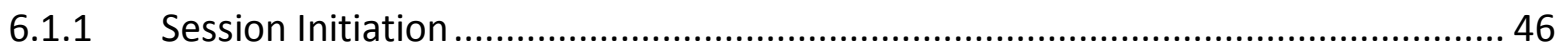

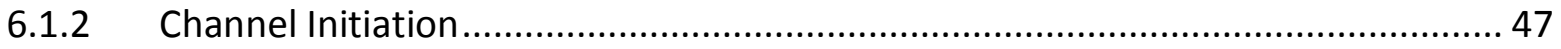

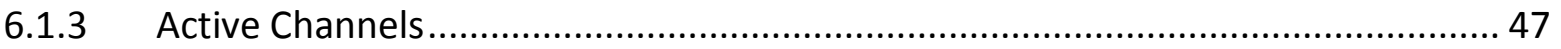

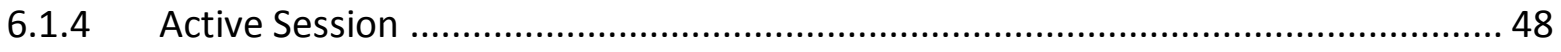

6.1.5 Channel Termination ..................................................................................... 48

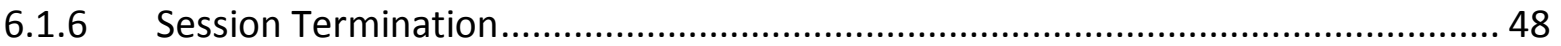

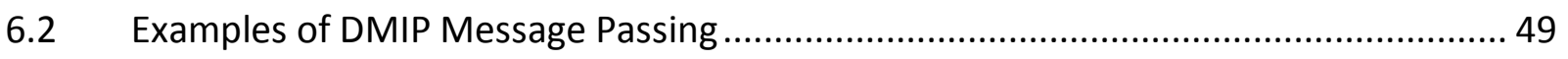

6.2.1 Client Request Session .................................................................................... 49

6.2.2 Adding Channels and Meta-Channels................................................................ 50

6.2.3 Sample Session, Button Based Menu …………….......................................... 52

6.2.4 Client Remove Abilities ...................................................................................... 54

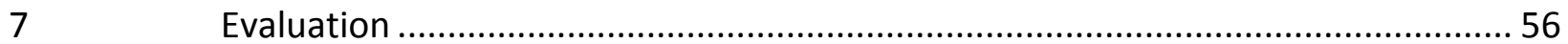

$7.1 \quad$ Validity of Channel Types System ............................................................................. 56

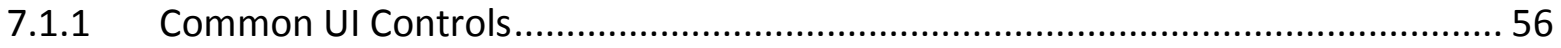

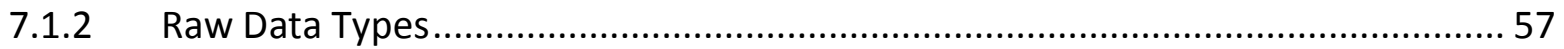

7.1.3 Bernsen's Multimodal Interface Modes …………………………………....... 57

7.1.4 Interaction Ledger.............................................................................................. 58 


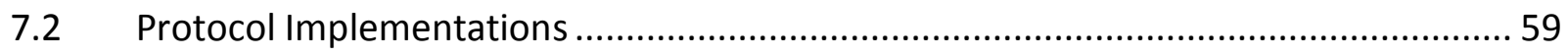

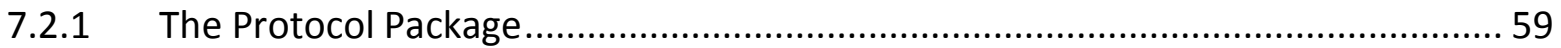

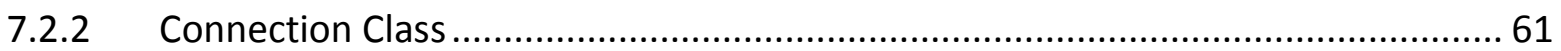

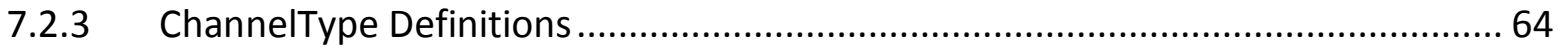

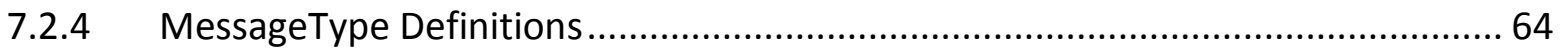

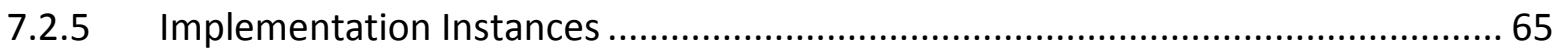

7.2.6 Protocol Support and Augmentation ..................................................................... 74

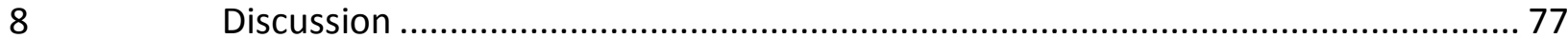

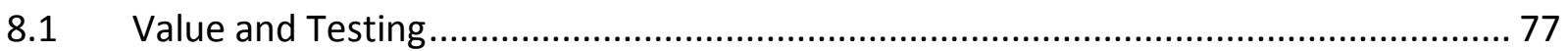

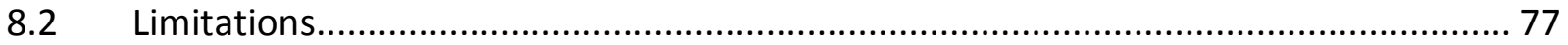

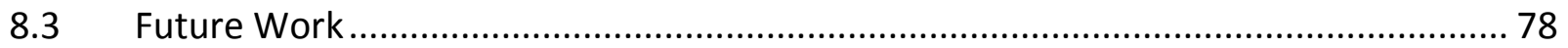

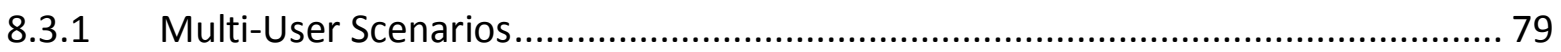

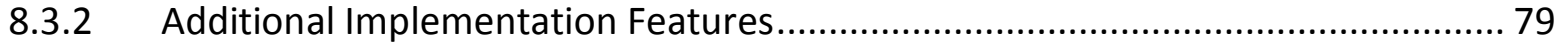

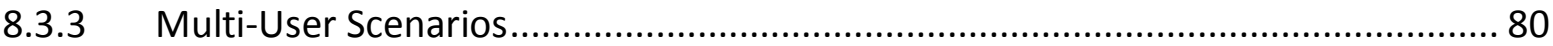

8.3.4 Integration with Other Technologies.................................................................. 80

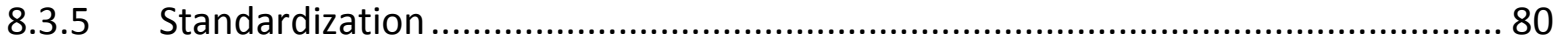

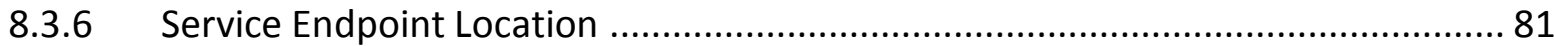

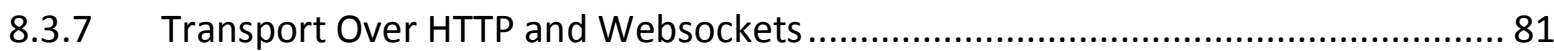

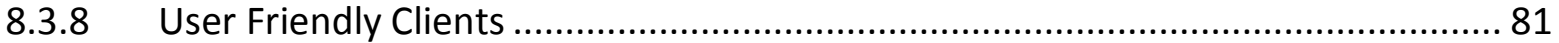

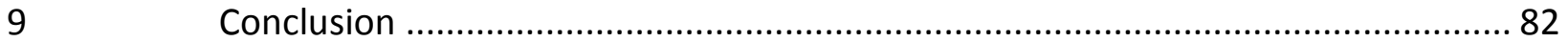

References 84

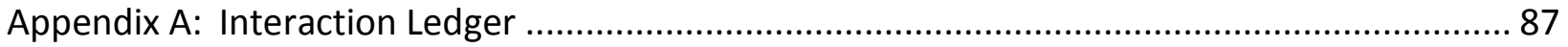

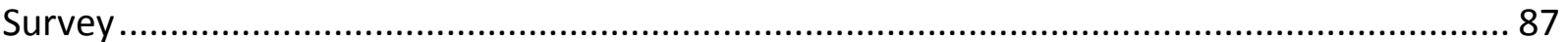

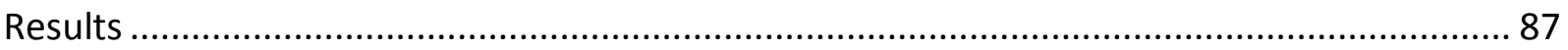

Appendix B: PowerPoint Controller Show XML ……............................................................... 92 


\section{List of Tables}

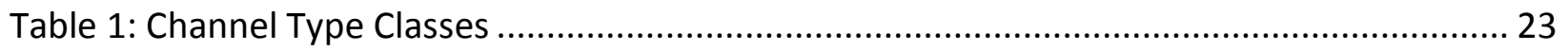

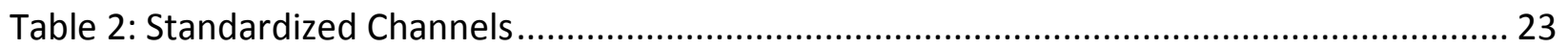

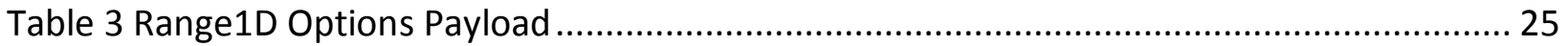

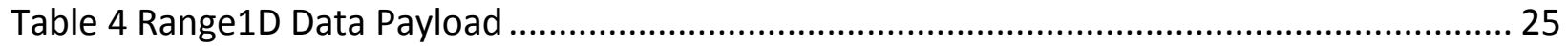

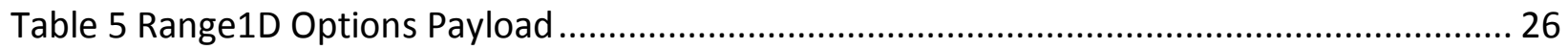

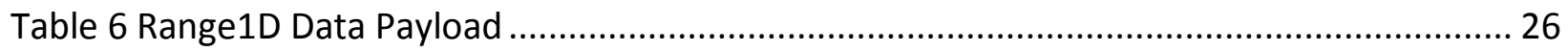

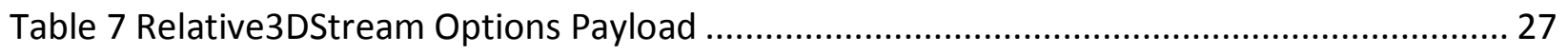

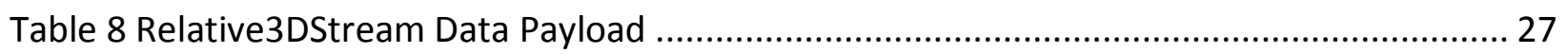

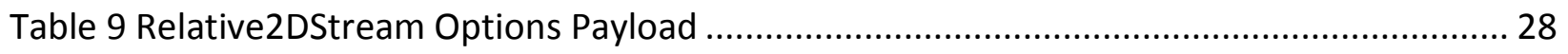

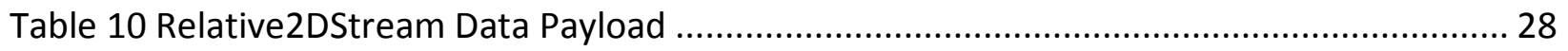

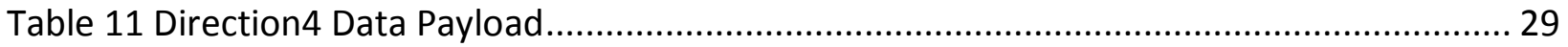

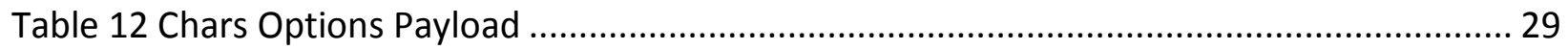

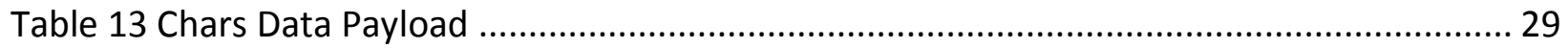

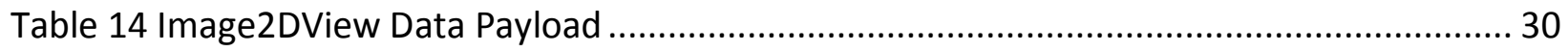

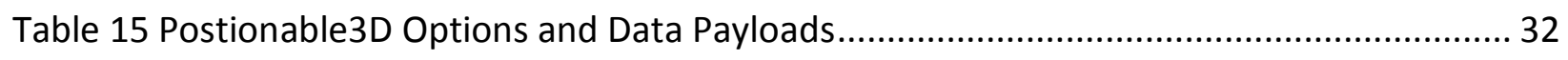

Table 16 Interactable Interaction Types and Argument Types .......................................... 32

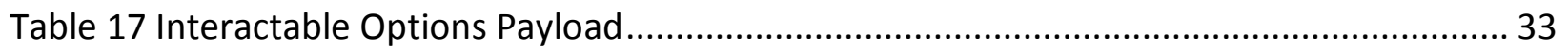

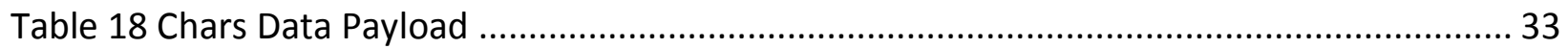

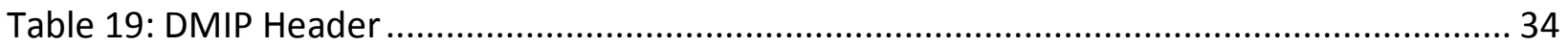

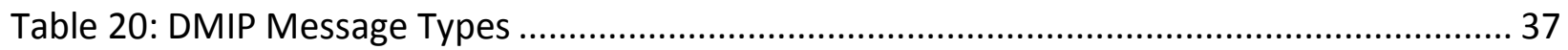

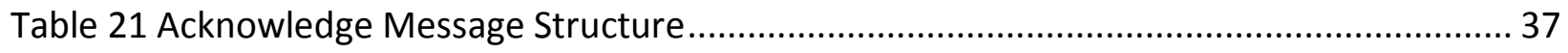

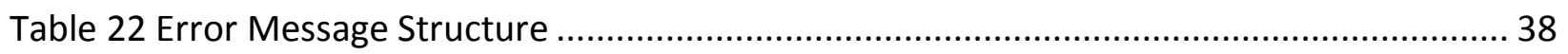

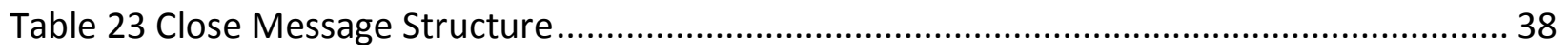

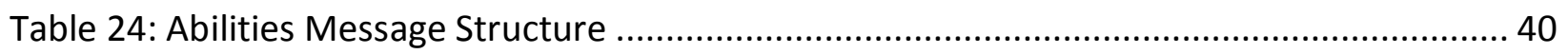

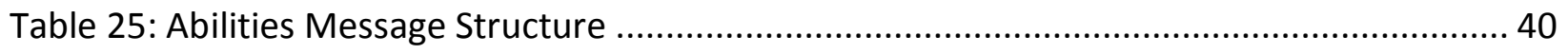

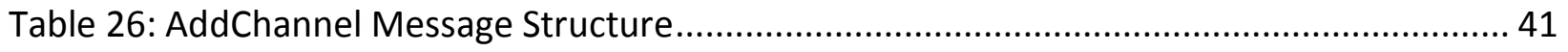


Table 27: RemoveChannel Message Structure

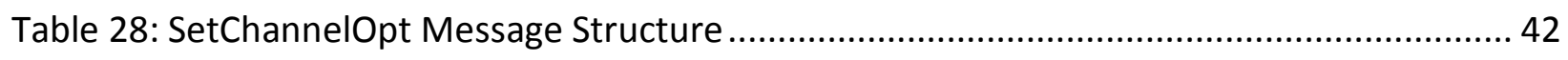

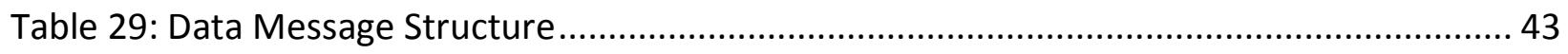

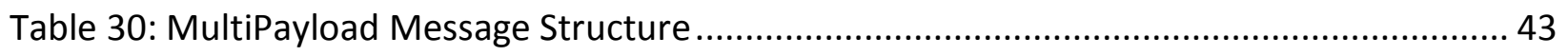

Table 31. Implemented DMIP Channel Types ................................................................... 66 


\section{List of Figures}

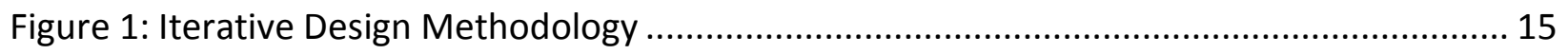

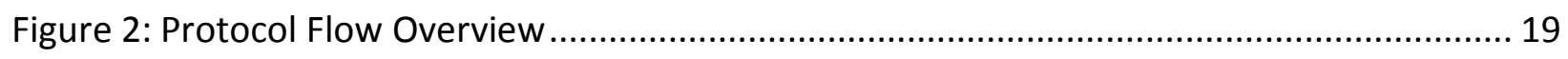

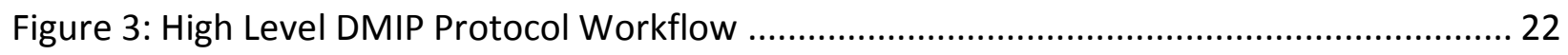

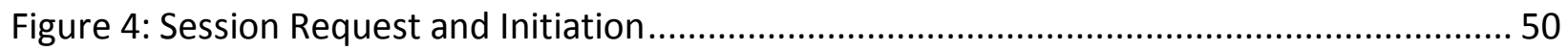

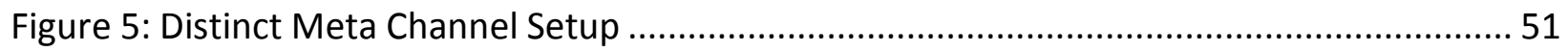

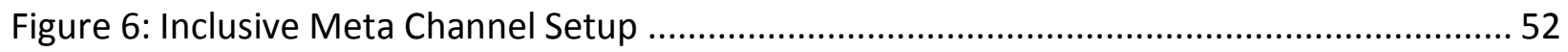

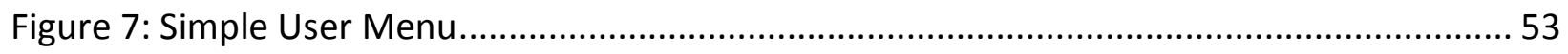

Figure 8: PowerPoint Controller Menu to Show Layouts ...................................................... 54

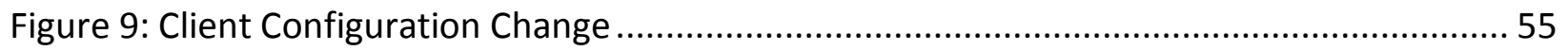

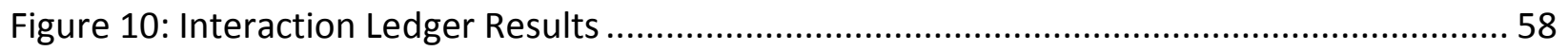

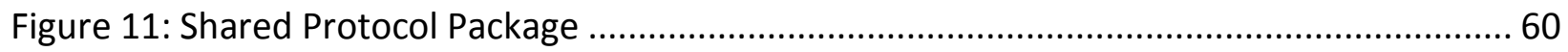

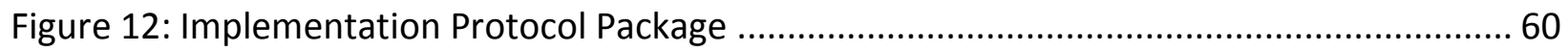

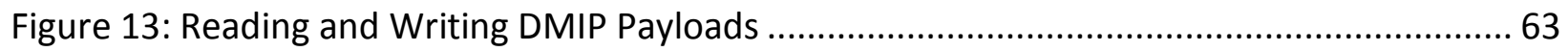

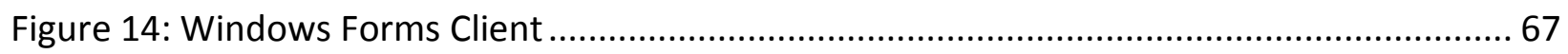

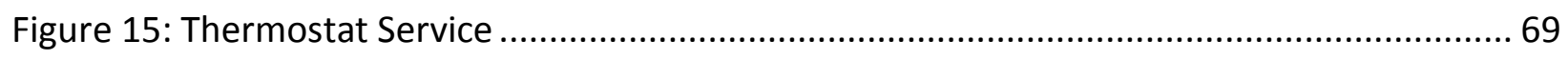

Figure 16: PowerPoint Controller from Android ............................................................. 70

Figure 17: Network Sequence for Previous Slide........................................................... 71

Figure 18: Number Guess Service Progression .................................................................... 72

Figure 19: Windows Mouse Control Service, Android Client ................................................. 74

Figure 20: Sample XML Layout File Result .................................................................. 76 


\section{List of Appendices}

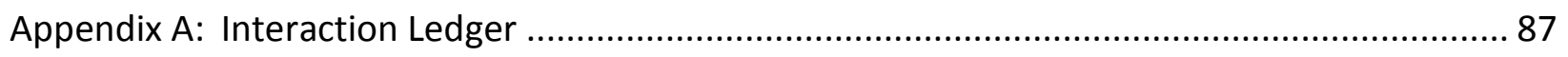

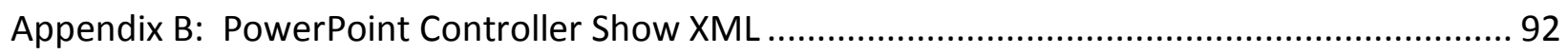




\section{Abbreviations and Terms}

\section{- DMIP: Distributed Multimodal Interaction Protocol}

The technology that is the subject of this thesis. An application protocol, using the Internet protocol suite that allows flexible data types to be negotiated and communicated.

\section{- Modality}

Refers to the type of communication channel used to convey or acquire information. It also covers the way an idea is expressed or perceived, or the manner an action is performed [1].

\section{- DMIP Abilities (Abilities)}

Refers to a listing of a DMIP client's abilities. It includes what display layouts it supports as well as an indication of which interaction modes it supports (by way of Channel Types, see 5.5) as well as the number of instances of an interaction mode it supports.

\section{- Mode}

Refers to a state that determines the way information is interpreted to extract or convey meaning [1].

\section{- $\quad$ Multimodality}

The capacity of the system to communicate with a user along different types of communication channels and to extract and convey meaning automatically [1].

\section{- TCP/IP: Internet Protocol Suite}

TCP/IP is a protocol set and model for inter connection between computers it provides end-to-end connectivity specifying how data should be formatted, addressed, 
transmitted, routed and received at the destination. TCP/IP has 4 distinct, cumulative layers: The link layer provides connection technologies for network segments, or links. The internet layer allows the interconnection of segments to provide internetworking. The transport layer provides protocols for communication low level octets between network endpoints. The application layer provides protocols that provide specific data communication over the underlying layers [2]. DMIP is an application layer protocol.

\section{- IP: Internet Protocol}

The IP protocol, or Internet Protocol is the networking layer in the Internet protocol suite that represents destination addresses and routing information for transmissible data [2]. It is the base protocol upon which TCP, UDP and other TCP/IP transport layer protocols are extended.

\section{- TCP: Transmission Control Protocol}

TCP is a TCP/IP transport layer protocol that provides reliable, ordered, error-checked delivery of a stream of octets between programs running on computers connected to a local area network, intranet or the public Internet [2]. The primary transport mechanism for DMIP.

\section{- UDP: User Datagram Protocol}

UDP is a TCP/IP transport layer protocol that provides a low-overhead and low latency transport mechanism, that does not provide error checking or receipt order verification [2]. UDP is a secondary and optional transport mechanism for DMIP, ideal for latency sensitive data streams.

\section{- Network Node or Endpoint}

These terms are both used to represent a network enabled device that can communicate over a TCP/IP network, and is therefore a potential DMIP capable device. 
- Data Type

A data type is strictly defined container for data. The general purpose of the DMIP is to negotiate compatible data types and allow communication of data encoded in the negotiated data types. Negotiable and transportable data types within DMIP are called Channel Types (5.5), and are used to communicate input and output mode data as well as pure data, such as text, video, images, etc.

\section{- $\quad$ Byte and Octet}

The terms byte and octet are used interchangeably within this thesis and represents an 8-bit value. Historically, byte was not consistent, so networks used the term octet. 


\section{Introduction}

Technology is everywhere within our homes, workplaces and places of leisure. The world is replete with computing devices, many of which are interactive, and networking of these devices has allowed basic interconnectedness among them. It is simple to envisage an environment where everything is controllable in a user-friendly way, from a personal device. However, using personal devices as general purpose controllers, providing human-usable methods of interacting with other, remote devices is not widespread. Technology that allows remote control has existed for well over a century for example, the remote control for your television. In the majority of cases these technologies do not work together, and when they do, the technology is often expensive, proprietary and requires the use of a single specific technology.

An example of a single controller interface that has been often demonstrated, but is not widely accepted is home automation. The concept is a simple one; use any device to control and manipulate the state of various devices in a home. Automatically turn off a light downstairs when you fall asleep, pre-heat the oven on the way home from work, change the TV channel from your phone, and monitor room temperature and security systems from afar. These home automation tasks are all common tasks that are technically feasible and appealing to end users and pieces of these exist [3] [4], and seem to work well. However, most of these solutions do not work together, and rely on costly application and networking design and development time because a common open standard for modal device to device communication is not available and are not widely implemented or adopted. The primary goal of this work (Distributed Multimodal Interaction Protocol: DMIP) is to allow intercommunication between devices using a standards-based open protocol to transport user interactions between endpoints. In this way, users can control their thermostat, TV, stove, or any other network connected device, from any other single networked device that is able to provide a sufficient user interface. Put simply, a user cannot currently use one general device to utilize the functionality of many others. This 
work describes the effort made in designing and creating a mechanism that allows interaction data to be remotely negotiated and transported.

DMIP aims to be able to provide a remote interface for any implementing devices. DMIP classifies interaction and data so that it can be transported over the network in a standard way. There is no open standard protocols that allow the transport of an expandable set of welldefined data types that represent the information needed to provide a human-centric, interactive experience. Providing a standard network protocol will enable more devices to inter-communicate by reducing costs associated with developing externally/remotely interactive devices. Some further examples of enabled scenarios are:

- Users enter a shopping mall and use any phone to interact with a large display to: play games with other shoppers, see specials, use interactive maps, and plan shopping routes.

- At a sports event, use the scoreboard display to play event-community games, polls, and contests; engage the audience with reduced technology investment.

- Use kiosks from personal devices, reduce the need for users to worry about interacting using the kiosk's touchpad and/or keypad, queue up actions before physically using the device reducing queue time.

The availability of an increasing number of types of input modes on personal devices like mobile telephones, music players and video game controllers as well as specialized input mode devices, like gaze trackers, motion trackers and brain-computer interface $(\mathrm{BCl})$ devices means that users have an ever increasing number of ways of interacting. In addition to supporting many interaction modalities, user focused design for interaction integrates interaction feedback to aid the user's understanding of the impact of their actions [5]. This work intends to provide an open standard method for negotiating and communicating both emerging and existing interaction mode data between network connected devices. To support the goal of this work, 
an open standard was designed to aggregate, negotiate and communicate a variety of static and interaction mode data signals so that they can be used by any platform adhering to it. This removes reliance on specific applications or platforms and only requires an interpreter application to be made available for client platforms. The intended result is a homogenous application platform the can be used by application creators to provide a more accessible access to technology. This decoupling of platform and data is accomplished through the introduction of an application layer network protocol, distributed multimodal interaction protocol (DMIP).

\subsection{Contributions}

This dissertation contributes to the human computer interaction field by providing a free and open standard networking protocol, Distributed Multimodal Interaction Protocol (DMIP) that allows classified interactions and data types to be negotiated and subsequently transported between two network nodes. Substantial effort has been put into creating a well thought out mechanism to transport all potential modal interaction data in a variety of situations and scenarios.

To support the understanding and adoption of DMIP, a number of resources have been made available, and discussed herein.

- The DMIP protocol specification

- Working implementations

○ Two DMIP network layer implementations, .NET framework and Java for Android

- Two DMIP client application implementations, .NET Windows Forms and Java for Android

- A DMIP service SDK for the .NET framework

○ Three DMIP service implementation examples, using the above SDK, for the .NET framework 
- An introductory conference paper, presented and published as part of the International Conference on Multimedia and Human Computer Interaction, 2013.

- A presentation made to, and discussed with the CapCHI society of Ottawa.

A website, (http://iv.csit.carleton.ca/ dmip/) has been created to host the protocol specification, above listed resources and any additional future resources, updates or discussions created to support DMIP and any future revisions of the protocol. In addition, the website will be a central source of information about new, standardized Channel Types, as discussed in 5.5.1. 


\section{Background}

The basic premise of computer networking is to enable computing devices to intercommunicate [6]. What information is communicated has largely been focused on computer formatted data, high level abstractions of human knowledge and actions. Focusing on the communication of human interaction elements allows the field of human computer interaction to be more directly applied to the manipulation of distinct devices. This could allow applications to be built that make use of a variety of interaction modes, giving application authors more ability to freely design services that make use of alternate modes much more simply. Enabling human users to use computers using their preferred input modes and devices. Similarly, providing a variety of options for system feedback (output) allows a broader range of users and user scenarios to be considered [7]. By providing more interaction options to the users to interact with remote devices, accessibility to those devices and the services they provide is increased [8]. Efforts to aggregate multimodal data exist, but are either not extendible, do not have a network transmission focus or are not open and freely usable. In section 2.2 Rationale, we discuss the need for this technology, from a point of technology ease of access evolution. Section 2.3 discusses reasons that such technology is not already widely available. In section 2.4 , we continue by examining related technologies and identify requirements for the proposed protocol to overcome the deficiencies in what exists today.

\subsection{Research Question}

There is an ever-increasing ability for the user to generate data on personal devices, can this data be simply consumed by remote computer devices to provide an interactive user based experience for potentially any capable remote technology?

\subsection{Rationale}

The answer to the question posed in 2.1 represents an evolutionary step towards simpler access to technology for end users. There are a number of reasons why it is needed to continue the world's population expanding access to technology. There exists an ever increasing use of 
server defined applications, via the World Wide Web (WWW) [9]. However to support these applications by providing improved usability and to make use of additional platform features on small form factor devices, like smartphones or tablets, specific clients for the large variety of hardware and software platforms are created and supported. This in turn generates a significant cost to develop, maintain and support these applications. Removing application logic and design from the client, so that a single application can be developed and maintained by application authors reduces costs and increases accessibility for users by removing the cost to create platform targeted applications and thus giving better access to the augmented application by way of providing for a greater number of supported client devices. For example, in a specific, single platform targeted scenario, there are at least two applications that need to be designed and developed, a client application and the service itself. Revisions of the functionality require careful consideration of client software. This issue is exacerbated when there are multiple platforms (example: Android, iOS, Blackberry, Windows Phone) and even further when considering variations in platforms, as well as the specific client device (abilities). A solution must remove the additional development time caused by dealing with multiple client targets, and removes the burden of carefully verifying every target device with every revision of an application by only having one functional piece for application authors to design, develop and maintain.

For end-users this is also partial solution to the problem of dealing with ever increasing numbers of specific applications on their mobile devices, making it difficult to locate and use them.

\subsection{Barriers}

While the technology herein is needed and has relevant rationale, prior to this work there did not exist a sufficient solution. A number of related technologies are discussed in 2.4 , but in general they do not fulfill the requirements needed to fulfill the goals of a distributed 
multimodal protocol. The reasons a prior solution did not exist is presumed to be a combination of 2 main factors:

- Using modes of interaction, besides mouse/keyboard are relatively novel

- Research efforts have created only protocols for very specific combinations of modes.

O Using alternate, not specifically defined sets of interaction modes has not been addressed.

- Efforts to increase product line value by providing applications

- Generate specific product line applications meeting only the temporal goals of the specific product line.

- Proprietary and not released or supported for external development.

- No perceived value to a production company for creating a standard to support devices or services produced by competing companies.

\subsection{Related Technologies}

Transporting user interactions over networks is a complex issue impeding the more pervasive spread of general human control of computers. There is a constant stream of new ways users can interact with technology [10], as new interaction technology is introduced it adds to the options available to application authors. Authors are confronted with design decisions that can limit the adoption of the new technology, or alienate users who do not have access to it. Furthermore, every application that makes use of the interaction data must encode data so that it can be transformed into input actions or output signals. Disparate projects have different goals and due to the proprietary nature of most of these works, the codification is not typically useable universally between applications. There are some existing technologies that attempt to simplify the transport and/or classification of multimodal data. Briefly, those most related to our work are as follows: 
- Tangible User Interface Objects (TUIO) [11] is a spatial (touch, motion tracking) input library that aims to aggregate these types of devices for use as an API.

- Responsive Objects, Surfaces and Spaces (ROSS) [12] provides a development toolkit for managing combinations of specific devices over a network.

- $\mathrm{HCl}^{2}[13]$ is a tool for aggregating machine-local input, so they can be more uniformly processed.

- Extensible MultiModal Annotation (EMMA) [14] is an extensible markup language (XML) W3C standard for encoding multimodal inputs.

- OpenNI [15] is a tool that is aimed at processing and aggregating motion based inputs.

- Streamlnput is a project aimed at providing a flexible input API.

- Radio Frequency for Consumer Electronics (RF4CE) [16] is a network protocol using low power links to create a decentralized "mesh" network.

- OpenRemote (http://www.openremote.com/) is a platform that focuses on home automation and provides solutions for managing existing proprietary and fixedinteraction home automation protocols.

- Application specifics Projects - that separately implement the parts of functionality required by the answer to the research question, but cannot work with one another because their implementations are non-standardized and used fixed mode-sets.

These are partial works, but are not sufficient to provide an extensible framework for reusable data type encoding and transmission. Next, we provide a detailed examination of each of these related technologies.

TUIO is a network based system that aims at providing aggregate touch input data. The purpose is to allow easier development of a variety of touch and other related continuous input systems [17]. While this technology is extensible [11] and does approximate some of the intentions of the proposed protocol, it is purely for input communication and does not have the ability to negotiate the usage of modes; implementations have fixed message expressiveness. 
Any application that needs to send data back to the client would not be directly implementable using TUIO. For example, a simple thermostat app might let you send input to adjust the temperature up or down, but the user would not be able to receive information about the current temperature. In addition, TUIO is designed purely for aggregating touch data, other interaction modes are not supported and negotiation of interaction modes, allowing variable sets of even touch interaction modes is missing. TUIO data is assumed to be touch based, and lacks the flexibility to simply define the data types needed for other interaction types. TUIO is therefore insufficient for providing a general interaction mechanism for network connected disparate devices.

ROSS is a development toolkit [12] for Tangible media [18] that provides a platform independent method of consuming and aggregating multimodal inputs. ROSS provides explicit input schema for devices connected to the system and operates over a TCP/IP network [12]. ROSS allows the development of applications that make use of specific devices. However, the need for explicit input schema/device templates for each endpoint limits the system's ability to provide services to new, emerging and varied device capabilities. For example, a client device might support 2D position data input using touch, mouse, gaze or through any number of other modalities, or combinations thereof. Further, the device may support selection tasks through varied modalities as well. In order for ROSS to interact with these devices, specific and fixed schema have to be developed for each device implementation.

\section{$\mathrm{HCl}^{2}$ : A Software Framework for Multimodal Human-Computer Interaction Systems}

describes a modular platform that aims to support the development and research of multimodal systems [13] [19]. This system provides a method of efficiently aggregating machine-local input signals, so that they can be processed. The framework is a research platform with a focus on performance, but there is no focus on raw data-type communication. $\mathrm{HCl}^{2}$ does not provide any networking facilities. Potentially the solution could use the aggregated local machine signals, provided by $\mathrm{HCl}^{2}$, to help communicate higher level user 
intent. As $\mathrm{HCl}^{2}$ is a software platform, the relation to a networking protocol is perhaps not obvious. However, its codification of input modes could be used on the service endpoint to help interpret user intent of a variety of inputs within a solution. It could also be used to define a specific method to carry $\mathrm{HCl}^{2}$ data, allowing endpoints to process signals for intent, and then communicating that intent. $\mathrm{HCl}^{2}$ by itself is insufficient to meet the distributed/remote aspect of a solution, however used in conjunction it could augment the other.

EMMA is an XML W3C standard [14] designed to alleviate the difficulty in aggregating and augmenting through annotation multimodal inputs by providing a standard for communicating them In XML format. EMMA is designed largely with speech audio data as the focus. An applicable solution protocol must have the potential to support both audio streams and text based data. However the overhead of EMMA's XML formatted data means that it is not well suited for a networked scenario. Additionally, the benefits of using a schema designed primarily for speech are minimal when considering the solution protocol's aims to be able to support the transport of most, if not all conceivable modalities. The solution protocol must provide a means to transport any type of modal data, potentially including EMMA's XML format.

Open $\mathbf{N}$ I is intended to provide an aggregation of processed continuous (primarily 3D vision) inputs [15]. It allows applications to be developed based on processed inputs from various sources. It does not provide a system for transport of these signals. A solution protocol must be able to operate over a network and provide the ability to transport a variety of modal data including potentially, 3D position and gesture data.

StreamInput is a project to create a cross-platform API to support application development for new sensors. Streamlnput is yet another system for aggregating interaction controls and does not provide any transport of such signals. This project is not yet released, but mentioned here as it may provide insight as to modes that should be supported in the future by the solution. Unfortunately, because StreamInput is not yet available, it is difficult to assume what it can and 
cannot it provide. However, seeing as the goals of the project are perceived to be similar to $\mathrm{HCl}^{2}$, Stream Input could likely provide the basis for the definition of a specific interaction mode, meant to carry StreamInput specific data.

RF4CE is a networking protocol designed to work over low power personal area networks to create interconnected "meshes" not reliant on a centralized network structure. RF4CE could potentially be used to find and connect to services, however it does not provide and explicit method of negotiating data types with disparate clients to define sets of modalities to use with clients. It provides a potential network connection option for the solution, but since the physical link layer is usually abstracted by application protocols and efforts are being made to provide a generalized TCP/IP abstraction (http://datatracker.ietf.org/doc/charter-ietf6lowpan/) for RF4CE.

OpenRemote appears to be a tool to enable aggregation and custom interfaces for other network protocols that are focused on home automation. This is an approach to solving the end users' problem of interacting with a variety of technologies from their personal device. However, since it relies of a fixed set of predefined modalities and data structures (from the aggregated protocols,) adaptability for new client devices and novel modalities is not present. A solution protocol must be able to represent a varying set of interaction modalities so that the users can interact with the features and abilities they, through their devices have.

Specific Applications are everywhere, there is a veritable glut of specific remote-control applications for phones and other handheld devices. Specific applications exist to control TV [3], PowerPoint Presentations [20], thermostat [4] and any number of other network-able devices. These types of applications have given users control over remote devices. However, there are two main issues with this "scheme" of creating specific applications. It is difficult to first interact with user-novel devices and development and maintenance of both endpoints is made extremely complex. 
All of the related technologies cited fall short of meeting the goals of a freely available standard for multimodal remote negotiation and transport.

To first interact a remote device that uses a specific application, a compatible application must be acquired. The user must know of, or be informed of the availability, have a method to acquire the application, and then install and configure it. These steps can all be problematic for users, notably if there is no information directly available about this function, there is no access to the internet from the client device or the device does not allow installation of applications. Furthermore, as the user acquires more specific applications, it is potentially difficult for the end user to locate or recall how to access the functionality for subsequent sessions. A solution provides applications as a network transported service, there are no requirements on the client for any application to be installed for a specific service, and only a single application should be required. A client needs only a network endpoint to connect to, something that could be facilitated using a service location mechanism like Service Location Protocol (SLP). 


\section{Protocol Design}

The need for a standard method of negotiating, defining and transporting multimodal data between disparate devices, is supported in section 2. By examining related technologies, barriers and the methods in which remote technology is currently accessed, a number of requirements for a new protocol; DMIP, were generated. Section 3.4Error! Reference source not found., Supporting Technologies introduces the technologies upon which the protocol is built.

\subsection{Target Audience}

The goal of this work is to provide a tool to simplify development of applications that communicate of variable set of interaction modalities. In turn, by providing a better tool for application authors, we aim to help the end-user have increased access to technology. The DMIP protocol, its specification (Section 5, Protocol) and associated resources (1.1 Contributions) are intended to be used by:

- Authors of

○ Client implementations

○ Service implementations

- Researchers intending to

Extend or revise the protocol

- Create derivative works

\subsection{Requirements}

Section 2, identified a number of requirements for an answer to the research question. Many of the related technologies deal with specific modalities or design-time fixed sets of modalities. Many of the works cited are not licensed for general use, and others still are verbose and not suitable for network transport. A formal list of identified requirements are compiled and clarified below; 
The system must:

i. Be format-flexible and have the potential to represent any type of computer data, especially raw format data and interaction modalities

ii. Provide a means to negotiate supported data-types between devices at run-time

iii. Be able to and depend upon operation over a computer network

iv. Be able to send and receive data asynchronously

v. Be able to provide access to any modal-compatible service through a single application

vi. Be able to provide a single, flexible platform for application developers

\subsection{Methodology}

Iterative design [21] a methodology frequently used in the $\mathrm{HCl}$ field was used during the development of the protocol specification, a diagram of the methodology is provided below in Figure 1: Iterative Design Methodology. Initially DMIP was designed based on requirements i, iii, iv, $v$ and vi. Then client and service implementations were created using the .NET framework (see section 7.2 Protocol Implementations). These implementations were evaluated and the protocol was revised to support (ii) and tested, subsequent iterations added modes, further services test and augmentations as well as introducing the Android platform. 


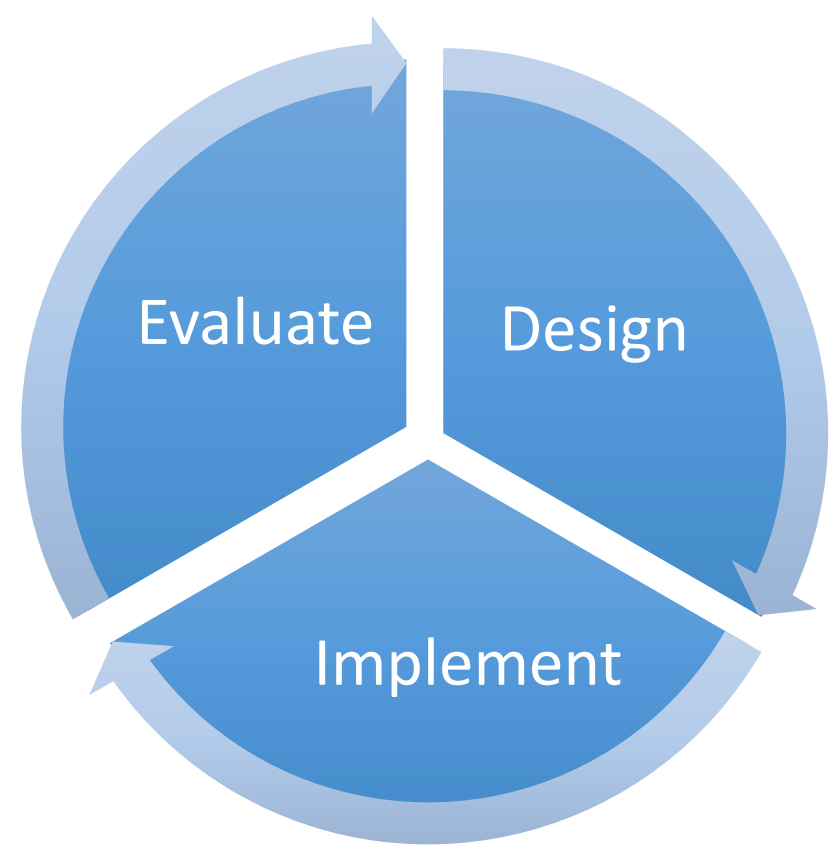

Figure 1: Iterative Design Methodology

\subsection{Supporting Technologies}

The DMIP protocol is built upon other foundational technologies, and assumes and extends the functionality therein. The Internet Protocol Suite (TCP/IP) is used to provide a communication medium. Service location Protocol (SLP) is a TCP/IP protocol that can be optionally used to make DMIP more accessible to human users by providing information about accessible DMIP endpoints.

TCP/IP: DMIP is designed to function on a TCP/IP (Internet Protocol Suite) stack network. The TCP/IP stack is designed to decouple reliance on the other network layers, DMIP is an uppermost TCP/IP stack layer protocol. The DMIP application layer protocol that makes explicit use of both Transmission Control Protocol (TCP) and User Datagram Protocol (UDP) transport protocols. Using the layers of the TCP/IP stack reduces any concern of link layer (physical transport medium) and can be transported using a large and expanding set of media.

The transport protocol TCP, is used for all aspects of the protocol except continuous data streams, which are transported using UDP. TCP provides a guarantee of in order packet delivery 
and is used primarily to ensure in order message delivery and processing, while UDP is used for continuous data streams, where a dropped or out of order message is less significant than the timeliness of message delivery [2]. The TCP/IP networking stack is broadly supported over a variety of application platforms and network media including wired and wireless [22] [23] technologies [2].

Service Location Protocol (SLP) [24]: DMIP is intended to be used in unfamiliar networks. DMIP applications assume a TCP/IP link and a TCP/IP endpoint are known. The TCP/IP link is generally configured by the user through their device, or automatically through applications on the device. DMIP endpoints must however be identified so that devices can connect. In order to provide a means of locating these DMIP endpoints, SLP is recommended as a solution. This protocol allows the discovery and query of managed service endpoints through the use of IP multicast. Further to identifying DMIP endpoints, basic attributes can be queried enabling connecting devices to connect only to compatible endpoints. The query-able attributes are yet to be defined and will be defined in future work by creating an SLP service template for DMIP [25]. 


\section{Technology Overview}

On a TCP/IP network communication occurs primarily over an endpoint to endpoint link [26]. These two endpoints can typically send and receive data in the form of octet encoded data from the other endpoint, using any number of supported transport layer protocols [2]. The most common transport protocols, TCP and UDP both allow the transport of octet streams in chunks. TCP provides a reliable mechanism with in-order guaranteed delivery of data while UDP provides a lower latency, but does not guarantee delivery or order of delivery [2]. The DMIP protocol uses primarily TCP connections, in order to provide a reliable end-user experience. UDP may be optionally used for the transport of some time-sensitive data if it is supported by both endpoints. DMIP is an application layer protocol that builds on transport layer protocols, primarily TCP and optionally UDP. Within a DMIP session there are always 2 endpoints (necessitated by TCP). One of these endpoints is a client (section 4.1), a DMIP implementing application that connects to and negotiates a session with a service (section 4.2), a DMIP application that "serves" the client the interface and can send and receive data using negotiated channels. Both clients and service devices can support multiple simultaneous sessions.

\subsection{DMIP Clients}

DMIP Clients are typically mobile devices that provide a remote, user-centric interface for a service. Client implementations do not contain any service application specific logic or presentation information. Clients initiate DMIP sessions by contacting a known network endpoint over TCP. The discovery of the addresses of these endpoints can be facilitated nicely using Service Location Protocol (SLP) [24].

Client DMIP applications are independent of services, a client that provides the minimum channels required to access a service can consume that service. Similar to a web browser, a single client implementation may be developed for a hardware device/platform, a user would not generally need more than one client on their device. However, client implementations 
could provide additional features by providing automatic service location, additional Channel Types (section 5.5) or any number of other options. It is therefore imaginable that multiple, potentially competing, clients for a platform might be developed.

\subsection{DMIP Services}

A DMIP service application is the functional piece. It is located at a known, advertised, or discoverable network endpoint. Interaction sessions using DMIP have all program logic and functionality, defined by the service application. To develop a DMIP service a developer creates client layouts and behaviours to support different modes of interaction by the service application.

\subsection{DMIP Channels}

DMIP Channels carry data, formatted according to the enclosing Channel Type (section 5.5) and represent input and/or output data from either endpoint. As shown in Figure 2: Protocol Flow Overview, a client sends a listing of its Abilities, represented by the Channel Types it supports (Label A). Then the service determines if it can interact with all, or a subset, of the presented abilities, and what channels it will use (Label B). Instances of the chosen channels are created (Label C). Once confirmed, a channel allows mode formatted data of that channel's type to flow (Label D). This data channel stream is valid until the connection times out (section 5.5), or is explicitly removed (section 5.6.2.8), Label E. 


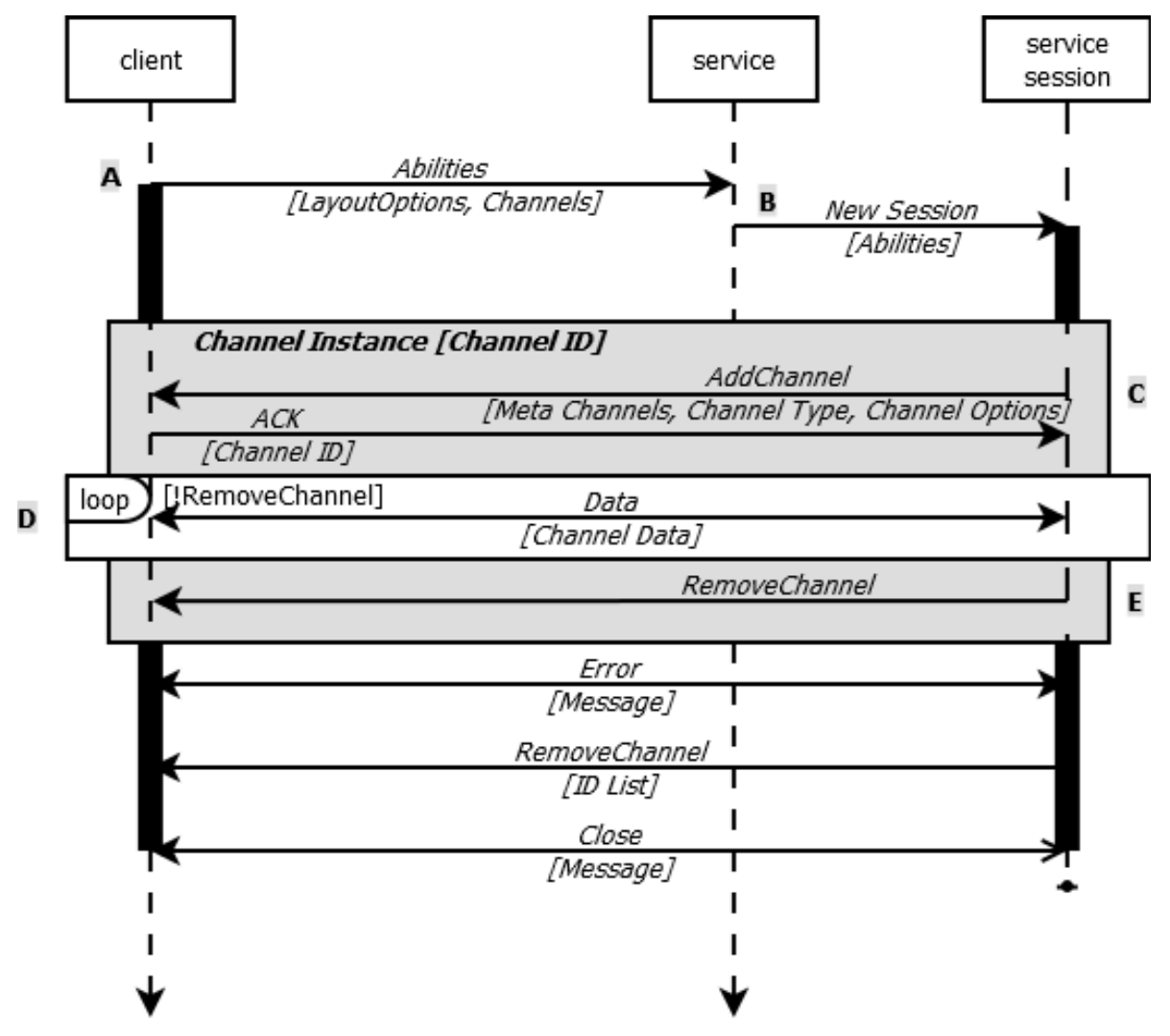

Figure 2: Protocol Flow Overview 


\section{Protocol}

The DMIP protocol intends to provide a standard method to negotiating and communicating both emerging and existing interaction modes to network connected devices.

\subsection{Protocol Applicability Statement}

DMIP is designed to function on a TCP/IP stack network. The DMIP protocol operates under the premise that a TCP/IP network endpoint is known, that is, that DMIP clients have acquired the IP address of service(s) to connect to. DMIP is an application layer protocol that makes use of both Transmission Control Protocol (TCP) and User Datagram Protocol (UDP) transport protocols. TCP MAY be used for all aspects of the protocol. However for timeliness, continuous data streams MAY be transported over UDP if so desired, and both endpoints support it. TCP provides a guarantee of in-order packet delivery and is used primarily to ensure in-order message delivery and processing, while UDP can be used for continuous data streams, where a dropped or out of order message is less significant than the timeliness of message delivery. UDP may be desirable for highly interactive applications using sensor data such as accelerometers.

\subsection{Terminology}

- Packet - A packet is a single TCP/IP message.

- Message - A DMIP formatted packet, must always contain a DMIP header, optionally followed by message data.

- Payload - A single DMIP message/instruction, these can be split or combined between multiple/single messages.

- Request - Synonymous to Payload, typically used as an alternative, for clarity, when an action by a remote endpoint is requested.

- Channels (or Channel type) - A static definition of a single type of transportable mode data. 
- Standard Channels - Channels included as part of the DMIP protocol definition (this document).

- Meta Channels - Channels used to augment other channel types, and can be associated 1 to 1 .

- Extension Channels - Channels created by $3^{\text {rd }}$ party and/or in development stages, not included within the protocol definition.

- Channel (or Channel type Instance) - An instance of a channel type, within an active DMIP session.

\subsection{Notation Conventions}

The key words "MUST", "MUST NOT", "REQUIRED", "SHALL", "SHALL NOT", "SHOULD", "SHOULD NOT", "RECOMMENDED", "MAY", and "OPTIONAL" in this document are to be interpreted as described in RFC 2119 [27].

If unspecified, binary data fields are to be interpreted as unsigned values.

\subsection{Protocol Overview}

The DMIP protocol provides a lightweight layer for communicating categorized interaction mode data over a TCP/IP network. DMIP operates over TCP, and optionally UDP. The purpose of the DMIP protocol is to provide functionality that simplifies the transport of interaction mode channels. The purpose of the creation of this protocol is to enable the possibility of making the devices around us to universally accessible This goal is accomplished by making a reusable and extendible protocol that simplifies development of distributed, network based applications, as it allows developers of service applications to focus on behaviours and presentation rather than being concerned with networking nor the interpretation of a wide range of input/output types over a range of platforms (OS/hardware). DMIP sessions are designed to be event based. The implementing systems observe and respond to events, which are generated by implementing systems when DMIP data is available. 
DMIP sessions are 1 to 1 ; a client initiates a session with a service by sending an Abilities packet (Abilities Message). Clients are independent of services, meaning a single client implementation of DMIP can connect to, and provide interactive experiences for a variety of DMIP services. Below, in figure 1, is a high level overview of the DMIP protocol in a system. A Client, (DMIP Clients, 4.1), is a standardized endpoint that is created and developed for a specific device or platform, a valid implementation supports any number of the standard DMIP channels (section 4.3) and can optionally support Meta channels (section 5.5.5). A service drives the application, in that it provides the layout and behaviour for clients. A protocol interpreter layer, such as that described in (section 7.1), allows multiple applications to be simply developed upon a single platform.

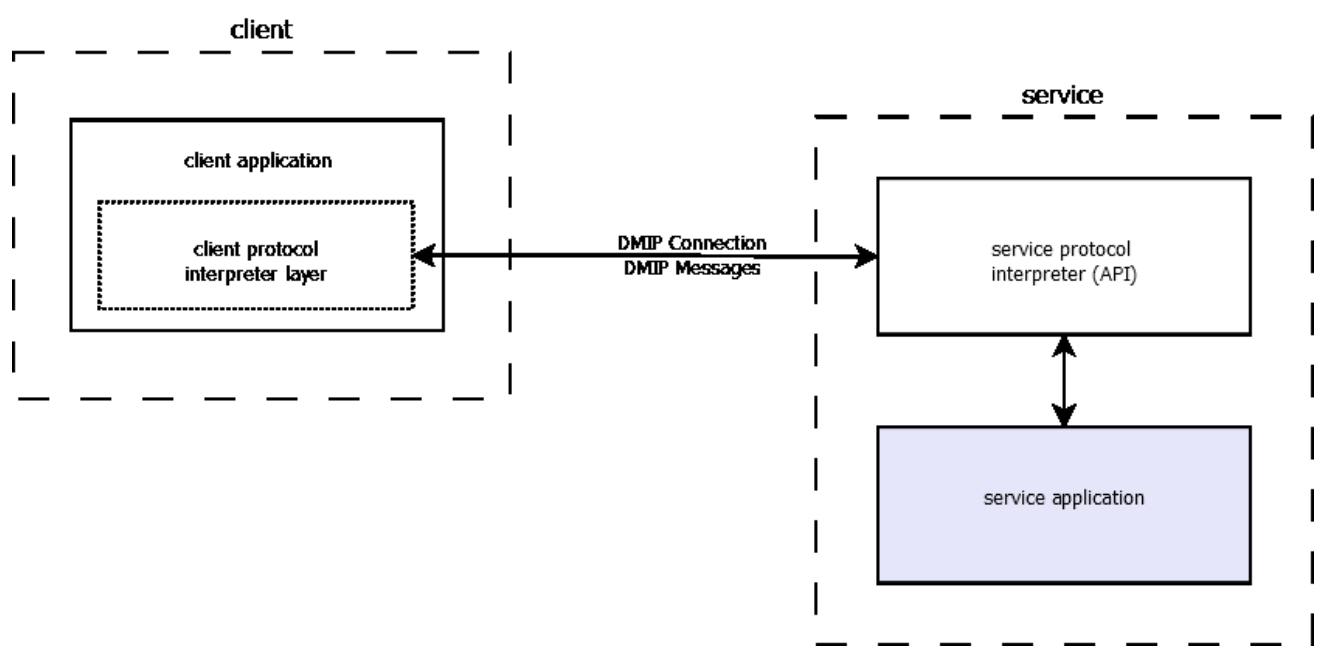

Figure 3: High Level DMIP Protocol Workflow

\subsection{Channel Types}

Channel Types represent a single type of mode data, and define what data can be transported within a Channel, which is an instance of a channel type. A client sends the channel types it can interpret, as part of Abilities payload. Channels are uniquely identified by 32-bit IDs.

Standardized channels fall within the values 0-1047 (11-bit). The standardized space is further subdivided, with the first 256 ( 8 bits) values reserved for meta-channels, 5.5.5. The remaining 
values (21-bits) 1025-4194304 can be used for extension channels, 5.5.5.4. Table 1: Channel Type Classes outlines the enumeration of the various classes of Channels.

Table 1: Channel Type Classes

\begin{tabular}{|l|l|}
\hline Channel Type Class & Range \\
\hline Standardized Meta Channels & $0-255$ \\
\hline Standardized Channels & $256-2047$ \\
\hline Extension Meta Channels & $2048-131071$ \\
\hline Extension Channels & $131072-4294967296$ \\
\hline
\end{tabular}

Table 2: Standardized Channels, below Lists the currently standardized channels.

Table 2: Standardized Channels

\begin{tabular}{|l|l|l|}
\hline Type ID & Name & $\begin{array}{l}\text { Description } \\
\text { An Empty Channel }\end{array}$ \\
\hline $\mathbf{1}$ & None & $\begin{array}{l}\text { Meta Channel: indicates the channel has a Unicode } \\
\text { text label }\end{array}$ \\
\hline $\mathbf{2}$ & PextLabel & Meta Channel: provides 3D vector positioning \\
\hline $\mathbf{3}$ & Interactable & Meta Channel: supports interaction events \\
\hline $\mathbf{4}$ & Enabled & $\begin{array}{l}\text { Meta Channel: allows a channel to be temporarily } \\
\text { "disabled" }\end{array}$ \\
\hline $\mathbf{2 5 6}$ & SingleState2DButton & A button \\
\hline $\mathbf{2 5 7}$ & Label2D & A label \\
\hline $\mathbf{2 5 8}$ & Range1D & A range slider \\
\hline $\mathbf{2 5 9}$ & ListSelect & A selection menu, with key value pairs \\
\hline $\mathbf{2 6 0}$ & TextBox & A text entry field \\
\hline $\mathbf{2 7 6}$ & Title & $\begin{array}{l}\text { A title, representing the caption of the client } \\
\text { application }\end{array}$ \\
\hline $\mathbf{2 9 6}$ & Relative3DStream & 32-bit, 3D data \\
\hline $\mathbf{2 9 7}$ & Relative2DStream & 32-bit, 2D data \\
\hline $\mathbf{3 1 6}$ & Direction4 & Direction data up/down/left/right \\
\hline $\mathbf{3 1 7}$ & Chars & Single character, various encodings \\
\hline $\mathbf{3 3 6}$ & Image2DView & 2D Image data, jpeg, png or bmp formats \\
\hline
\end{tabular}

\subsubsection{Channel Type Definition}

Channel types are defined by two pieces; channel type Options sent as part of an AddChannels or SetChannelOpt payload and channel type Data sent as the main content of a DMIP Data payload. Both of these pieces need to be implemented to define a Channel Type. 
Implementation of a channel type includes definition of what data is included, and how that data is to be encoded/decoded into message data.

\subsubsection{Channel Type Options}

The Options payload of a channel is sent to provide initialization and configuration information about the channel. An Options payload is sent to initialize a channel as part of an encapsulated AddChannel payload. If options for a channel need to be reset, or initialized for a Meta Channel they can be sent as part of a SetChannelOpt payload. Note that AddChannel messages are ignored for already configured channels. Receipt of a SetChannelOpt payload indicates that the channel, or Meta Channel should be reset to a default state with the Option payload data used to re-initialize it.

\subsubsection{Channel Type Data}

The channel Data payload is sent as part of a DMIP Data message. Data payloads include formatted data for the channel, and can be event based (discrete) or stream based (continuous). When developing new or extension channel types the importance of in-order delivery and the frequency of transmission should be considered, so that the Data payloads can be developed accordingly, features could be added to channel type Data definitions that are likely to be used over UDP to augment UDP support.

\subsubsection{Standard Channels}

\subsubsection{None}

The ChannelType enumeration value zero $(0)$ is reserved and should be ignored by endpoints implementing the protocol.

\subsubsection{SingleState2DButton}

The SingleState2DButton (256) channel is intended to be represented by a selectable button on clients. The channel type is a skeleton that is intended to have meta-channels attached to it. It is recommended to be implemented by a standard looking button according to the client implementation platform's standard user interface conventions, or as the developers sees fit. 
The channel's appearance, layout parameters and behaviours can be specified/augmented through the use of meta-channels. Both SingleState2DButton Options and Data are zero length.

\subsubsection{Label2D}

The Label2D (257) channel is intended to be represented by a text label on connected clients.

The channel type is a skeleton that is intended to have meta-channels attached to it. It is recommended to be implemented by a standard looking label according to the client implementation platform's standard user interface conventions, or as the developers sees fit. The channel's appearance, layout parameters and behaviours can be specified/augmented through the use of meta-channels. Both Label2D Options and Data are zero length.

\subsubsection{Range1D}

The Range1D (258) channel is intended to be represented by a range slider or appropriate alternative on connected clients. The channel type allows a bounded value to be selected by clients. The channel's appearance, layout parameters and behaviours can be specified/augmented through the use of meta-channels. 4-octet values of various types are supported. Signed and Unsigned 4-octet integers and floating point numbers are supported. Type values: 1: Unsigned Integer, 2: Signed Integer, 3: Floating Point. The Range1D Data payload should be sent when the channel's value changes on the client.

Table 3 Range1D Options Payload

\section{Channel Type: Range1D (258)}

\begin{tabular}{lll}
\hline Field & Size & Description \\
\hline Type & 8 bits & Represent the value type of the output \\
\hline Max & 32 bits & The maximum value that the channel can select, of Type \\
\hline $\begin{array}{l}\text { Min } \\
\text { Table 4 Range1D Data Payload }\end{array}$ & 32 bits & The minimum value that the channel can select, of Type
\end{tabular}

Channel Type: Range1D (258)

$\begin{array}{lll}\text { Field } & \text { Size } & \text { Description } \\ \text { Value } & 32 \text { bits } & \text { The value currently selected by the Range1D channel }\end{array}$




\subsubsection{ListSelect}

The ListSelect (259) channel is intended to be represented by a dropdown list or spinner that typically can display one line of text representing the currently selected value. The channel's appearance, layout parameters and behaviours can be specified/augmented through the use of meta-channels. 4-octet values of various types are supported. Signed and Unsigned 4-octet integers and floating point numbers are supported. Type is specified within a Range1D options payload; values: 1: Unsigned Integer, 2: Signed Integer, 3: Floating Point. By default, the Range1D Data payload should be sent when the channel's value changes on the client.

Table 5 Range1D Options Payload

\section{Channel Type: Range1D (258)}

\begin{tabular}{ll|l}
\hline Field & Size & Description \\
\hline Type & 8 bits & Represent the value type of the output \\
\hline Max & 32 bits & The maximum value that the channel can select, of Type \\
\hline Min & 32 bits & The minimum value that the channel can select, of Type
\end{tabular}

Table 6 Range1D Data Payload

\section{Channel Type: Range1D (258)}

$\begin{array}{lll}\text { Field } & \text { Size } & \text { Description } \\ \text { Value } & 32 \text { bits } & \text { The value currently selected by the Range1D channel }\end{array}$

\subsubsection{TextBox}

The TextBox (260) channel is intended to be represented by a text box on connected clients.

The channel type is a skeleton that is intended to have meta-channels attached to it, especially the TextLabel meta-channel to provide communication of text data that would typically appear in the textbox. It is recommended to be implemented by a common looking textbox according to the client implementation platform's standard user interface conventions, or as the client developer sees fit. The channel's appearance, layout parameters and behaviours can be specified/augmented through the use of meta-channels. Both TextBox Options and Data are zero length. 


\subsubsection{Title}

The Title (276) channel is intended to represent a window or application caption, typically shown in the title area of an application, and running application lists on clients. The channel type is a skeleton that is intended to have meta-channels attached to it. It is recommended that implementations implement at least the TextLabel Meta Channel, allowing a text label to be specified. Both Title Options and Data are zero length.

\subsubsection{Relative3DStream}

The Relative3DStream (296) channel is designed as client output channel, and would not usually be rendered. However, if representation were desired Meta Channels can be used. This channel is intended to represent relative 3D spatial data, primarily accelerometer data, but other data could be represented. Fields for the channel are to be interpreted as single-precision floating point format. This channel is intended to be used to send data continuously when data is available and is recommended to be sent over a low-overhead transport protocol, such as UDP. Interpretation of the data for the channel is left to client and service developer discretion. Below are the structure of the Options and Data payloads.

Table 7 Relative3DStream Options Payload

\section{Channel Type: Relative3DStream (296)}

\begin{tabular}{llll}
\hline Field & Size & Description \\
\hline MaxX & 32-bits & The maximum absolute value of the $X$ axis data \\
\hline MaxY & 32-bits & The maximum absolute value of the $Y$ axis data \\
\hline MaxZ & 32-bits & The maximum absolute value of the $Z$ axis data
\end{tabular}

Table 8 Relative3DStream Data Payload

\section{Channel Type: Relative3DStream (258)}

\begin{tabular}{|c|c|c|}
\hline Field & Size & Description \\
\hline $\mathbf{x}$ & 32-bits & Value of the $X$ axis data \\
\hline $\mathbf{Y}$ & 32-bits & Value of the $Y$ axis data \\
\hline $\mathbf{Z}$ & 32-bits & Value of the $Z$ axis data \\
\hline
\end{tabular}




\subsubsection{Relative2DStream}

The Relative2DStream (297) channel is designed as client output channel, and would not usually be rendered. However, if representation were desired Meta Channels can be used. This channel is intended to represent relative 2D spatial data, which could represent computer mouse movements, or a variety of other data. Fields for the channel are to be interpreted as singleprecision floating point format. This channel is intended to be used to send data continuously when new data is available and is recommended to be sent over a low-overhead transport protocol, such as UDP. Interpretation of the data for the channel is left to client and service developer discretion. Below are the structure of the Options and Data payloads.

Table 9 Relative2DStream Options Payload

\section{Channel Type: Relative2DStream (296)}

\begin{tabular}{llll} 
Field & Size & Description \\
\hline MaxX & 32-bits & The maximum absolute value of the $X$ axis data \\
\hline MaxY & 32-bits & The maximum absolute value of the $Y$ axis data
\end{tabular}

Table 10 Relative2DStream Data Payload

\section{Channel Type: Relative3DStream (258)}

\begin{tabular}{lll} 
Field & Size & Description \\
\hline $\mathbf{X}$ & 32-bits & Value of the $X$ axis data \\
\hline $\mathbf{Y}$ & 32-bits & Value of the $Y$ axis data
\end{tabular}

\subsubsection{Direction4}

The Direction4 (316) channel is designed as client output channel, and would not usually be rendered. However, if representation were desired Meta Channels can be used. This channel is intended to represent discrete, 4 direction data, and could be used to represent the arrow keys on a standard keyboard, a directional pad, or a basic joystick. The Options payload is zero length. When a direction is specified by the client, a Data payload is sent, with a single direction $(U p=1$, Left $=2$, Down $=3$, Right $=4)$ single octet field is sent. The structure of the Data payload is given below. Values outside the given enumeration should be ignored. 
Table 11 Direction4 Data Payload

Channel Type: Direction4 (316)

$\begin{array}{lll}\text { Field } & \text { Size } & \text { Description } \\ \text { Direction } & \text { 8-bits } & \text { The direction selected, } U p=1, \text { Left }=2, \text { Down }=3, \text { Right }=4\end{array}$

\subsubsection{Chars}

The Chars (317) channel is designed as client output channel, and would not usually be rendered. However representation were desired Meta Channels can be used. This channel is intended to represent discrete, character data, and could be used to represent keyboard input. The Options payload specifies the expected encoding of the character data $(\mathrm{ASCI}=1, \mathrm{UTF} 8=2$, UTF16 = 3, UTF32 = 4). Standard usage would include the client user pressing a key on a keyboard, resulting in a Chars Data payload being sent to the service, including the encoded character's value. The structures of the Options and Data payload are provided below. Undefined values should be ignored. Note that Data payloads also specify encoding, the Options payload is to be used as a client's intended format, however Services implementing the Chars channel type, MUST support all 4 encoding schemes.

Table 12 Chars Options Payload

\section{Channel Type: Chars (317)}

$\begin{array}{lll}\text { Field } & \text { Size } & \text { Description } \\ \text { Encoding } & \text { 8-bit } & \text { The intended encoding of the characters sent within Data payloads; } \\ & & \text { ASCII }=1, \text { UTF8 }=2, \text { UTF16 }=3, \text { UTF32 }=4\end{array}$

Table 13 Chars Data Payload

\section{Channel Type: Chars (317)}

\begin{tabular}{|c|c|c|}
\hline Field & Size & Description \\
\hline Encoding & 8-bit & $\begin{array}{l}\text { The encoding of the included character; } \mathrm{ASCI}=1, \mathrm{UTF} 8=2, \mathrm{UTF} 16= \\
3, \mathrm{UTF} 32=4\end{array}$ \\
\hline Character & $\begin{array}{l}\text { ASCII }=8 \text {-bit } \\
\text { UTF8 }=8 \text {-bit } \\
\text { UTF16 }=16 \text {-bit } \\
\text { UTF32 }=32 \text {-bit }\end{array}$ & A single character, encoded according the Encoding field. \\
\hline
\end{tabular}




\subsubsection{Image2DView}

The Image2DView (336) channel is intended to be represented by a view of an image on connected clients. Images can be represented in large octet streams, and split over more than one packet, it is recommended that TCP or other in-order guaranteed transport layer is used. The Options payload is 0 length, augmentation can be provided with Meta Channels. Behaviour with no meta-channels is undefined, but valid, client developers can utilize the channel as they see fit if no meta channels are used (write images to local storage, provide a full screen show, etc.) Image2DView supports JPEG $=1, \mathrm{PNG}=2, \mathrm{BMP}=3$, or other $=255$ encoded images, extended formats may be added in revisions to Image2DView. Implementations can try to decode any image format not within the given encodings, or with an encoding of Other, but should either generate a placeholder image, or show empty space if the image cannot be decoded. Data payloads include the image's width and height in pixels to aid in layout, should the image not be decode-able. Below is the format of Data payloads.

Table 14 Image2DView Data Payload

\section{Channel Type: Chars (336)}

\begin{tabular}{|c|c|c|}
\hline Field & Size & Description \\
\hline Encoding & 8-bit & $\begin{array}{l}\text { The encoding of the included image; JPEG }=1, P N G=2, B M P=3 \text {, } \\
\text { other }=255\end{array}$ \\
\hline Width & 16-bit & Unsigned 16-bit integer representing the image's width, in pixels \\
\hline Height & 16-bit & Unsigned 16-bit integer representing the image's height, in pixels \\
\hline ImageData & Variable & The encoded image binary data \\
\hline
\end{tabular}

\subsubsection{Meta Channels}

Meta Channels are channels used to augment other channel types. Clients capable of using a given Meta channel with a channel, specify so, within an Abilities payload. Services compatible with the Meta channel can specify a Meta channel's options with a SetChannelOpt message. Only one instance of each type of Meta channel is valid for a given channel. Please see the proceeding sections for examples, meta-channels reduce channel overhead by reducing the size of data messages and can provide aggregation of common channel functionality. 


\subsubsection{Textlabel}

The TextLabel (1) Meta Channel allows text strings to be associated to channels. For example, with the currently implemented channels, it can be used to provide the text for a button, application window or a label, or a caption for an image. Both the TextLabel Data and Options payloads have identical structures, and contain only a string, a series of UTF-16 characters.

\subsubsection{Positionable3D}

The Positionable3D (2) Meta Channel allows client-rendered channels, that are representable as user controls or widgets to be positioned in the application's layout. Both Data and Option payloads are identical. If a position is changed for an existing channel (using a Data payload), the transition is undefined, but should not impede usage of the channel by the client's user. Three dimensional values are used to provide ordering using the depth dimension on 2D displays, or to provide positioning in 3D environments. Positionable3D supports both scalar and vector co-ordinates, the former can be used to allow vectored positions to be calculated within the service using the dimensions provided as part of the LayoutOption within the initial Abilities message. Scalar positions are communicated in 32-bit integer values. Vector values are transported as 32-bit floating point values, from 0 to 1 representing the full range of the given dimension. Negative values or values beyond 1 (or beyond the display's maximum scalar value) are valid and could be used to place controls partially, or entirely off screen. Within a single payload, scalar and vector values cannot be mixed. Below is the structure of Positionable3D payloads. 
Table 15 Postionable3D Options and Data Payloads

Channel Type: Positionable3D (2)

\begin{tabular}{|c|c|c|}
\hline Field & Size & Description \\
\hline $\mathrm{X}$ & 32-bits & $\begin{array}{l}\text { The intended horizontal position (32-bit integer or signed floating } \\
\text { point) }\end{array}$ \\
\hline $\mathbf{Y}$ & 32-bits & $\begin{array}{l}\text { The intended vertical position (32-bit integer or signed floating } \\
\text { point) }\end{array}$ \\
\hline $\mathbf{Z}$ & 32-bits & The intended depth position (32-bit integer or signed floating point) \\
\hline Width & 32-bits & The width (32-bit integer or signed floating point) \\
\hline Height & 32-bits & The height (32-bit integer or signed floating point) \\
\hline Depth & 32-bits & The depth (32-bit integer or signed floating point) \\
\hline Scalar & 8-bits & $\begin{array}{l}\text { A Boolean representing if the fields are to be interpreted as integers } \\
\text { or floating point values ( } 0 \text { for integer/scalar, otherwise floating } \\
\text { point/vector) }\end{array}$ \\
\hline
\end{tabular}

\subsubsection{Interactable}

The Interactable meta-channel allows Channels to have 1 or more classical user initiated interactions attached to them. The Options payload allows specification of any number and combination of the defined interactions

Table 16) as well as an optional argument type for that interaction, that will defines the format of an optional argument transported when that interaction occurs on the Channel. The Data payload is transmitted when the interaction occurs, and contains the type of interaction (

Table 16) as well as an octet array containing a parameter in the format specified in Options when the channel was created (argument type,

Table 16).

Table 16 Interactable Interaction Types and Argument Types

Type Enumeration Values

Interaction 1: Select, 2: Focus, 3: Blur, 4: Change

Type

Argument None; 0

Type Unsigned Integer; 1: 8 bit, 2: 16 bit, 3: 32 bit

Integer; 8: 16 bit, 9: 32 bit

Character array*; 16: UTF8, 17: UTF16

Floating point; 24: 32 bit, 25: 64 bit,

Boolean: 32 : 8 bit;

*Character array argument types represent a string of characters in the specified format; UTF8 or UTF16. 
Provided below are the bit-layouts of the Options and Data Interactable payloads.

Table 17 Interactable Options Payload

\section{Channel Type: Interactable (3)}

Field Size Description

1...*: Octet Pair listing of interactions

Interaction 8-bit Type of interaction, See

Type Table 16

Table 18 Chars Data Payload

Channel Type: Chars (317)

\begin{tabular}{|c|c|c|}
\hline Field & Size & Description \\
\hline Encoding & 8-bit & $\begin{array}{l}\text { The encoding of the included character; } \mathrm{ASCII}=1, \mathrm{UTF} 8=2, \mathrm{UTF} 16= \\
3, \text { UTF32 }=4\end{array}$ \\
\hline Character & $\begin{array}{l}\text { ASCII }=8 \text {-bit } \\
\text { UTF8 }=8 \text {-bit } \\
\text { UTF16 }=16-\text { bit } \\
\text { UTF32 }=32-\text { bit }\end{array}$ & A single character, encoded according the Encoding field. \\
\hline
\end{tabular}

\subsubsection{Enabled}

The Enabled (4) meta-channel allows enabling or disabling any rendered control on the client. It is important to note that Enabled is purely a client interface feature and SHOULD NOT indicate that data for that channel is invalid. The Enabled meta-channel can provide helpful user interface cues when a feature is not available and SHOULD cause the client rendered control representing the underlying channel to appear disabled. Both the Enabled Data and Options payloads have identical structures, and contain only a single octet, with a value of 0 representing false (not enabled) and any other value representing true (enabled).

\subsubsection{Extension Channels}

Extension channels allow data beyond what is specified within standardized channels to be transported. Clients and services that implement extension channels must be capable of performing further verification of the channel type, through the channel type options data that is sent during AddChannel and SetChannelOpt messages from the service. 


\subsection{Messages}

A DMIP message is a formatted packet, made up of a DMIP header followed by message data.

\subsubsection{Header}

The DMIP Header is a 15 -octet (120 bits) grouping of data that is prepended and transmitted with every DMIP TCP or UDP message. Included in

Table 19: DMIP Header is a description, in byte order, the fields within the header.

\begin{tabular}{|c|c|c|c|}
\hline Bit Index & Field & Size & Description \\
\hline 0 & Length & $\begin{array}{l}16 \text { bits ( } 65535 \text { bytes } \\
\text { per message) }\end{array}$ & $\begin{array}{l}\text { Unsigned little-endian } \\
\text { representing the message octet } \\
\text { size }\end{array}$ \\
\hline 16 & Protocol version & 8 bits ( 256 versions) & $\begin{array}{l}\text { Aid in identifying potential } \\
\text { differing versions of DMIP }\end{array}$ \\
\hline 24 & Message type & $\begin{array}{l}8 \text { bits ( } 256 \text { message } \\
\text { types) }\end{array}$ & Identify the type of message \\
\hline 32 & Timestamp & 32 bits (49.7 days) & $\begin{array}{l}\text { Milliseconds elapsed on the } \\
\text { DMIP endpoint since the } \\
\text { session began }\end{array}$ \\
\hline 64 & Message ID & $\begin{array}{l}8 \text { bits ( } 256 \\
\text { simultaneous } \\
\text { messages) }\end{array}$ & $\begin{array}{l}\text { Avoid duplicate processing of } \\
\text { simultaneous messages }\end{array}$ \\
\hline 72 & TunnelID & $\begin{array}{l}16 \text { bits ( } 65536 \text { DMIP } \\
\text { connections per } \\
\text { TCP/IP port) }\end{array}$ & $\begin{array}{l}\text { Allows multiple connections to } \\
\text { be a carried over a single TCP/IP } \\
\text { port }\end{array}$ \\
\hline 88 & PacketCount & $\begin{array}{l}16 \text { bits ( } 65536 \text { packets } \\
\text { per message max) }\end{array}$ & $\begin{array}{l}\text { The number of packets in the } \\
\text { message }\end{array}$ \\
\hline 104 & PacketNumber & 16 bits & $\begin{array}{l}\text { The number of the packet } \\
\text { within the message }\end{array}$ \\
\hline
\end{tabular}

The length field is included first to simplify the delineation of messages from streams. It is included in both UDP and TCP messages in order to provide an identical header over both transport layers and potentially allow other transport layer protocols to be implemented with comparatively less difficulty. 
The protocol version will be 1 for the initial version of the protocol, and will be incremented if changes are made that are not directly backward and forward compatible.

TunnelID is used to allow the transport of data for more than one interface over a single connection. Messages using different TunnelIDs MUST be treated as part of an independent session/connection.

Message types are an enumerated type and indicate how the message data will be interpreted, message types and associated enumeration values are included in 5.6.2.

A timestamp field is used to allow messages from disparate clients to be synchronized on the host; an intelligent multi-client host may use this information to compensate for network latency. The timestamp can be used in conjunction with the Message ID to avoid duplicate processing of messages, for example from UDP data messages, or multi-homed messages. The message ID field should be unique for any given Timestamp.

The length field is used to allow split messages to be recombined correctly.

The PacketCount and PacketNumber fields allow messages to be split into smaller packets, this can be used if a network endpoint has a preferred maximum, or there is a maximum TCP or UDP packet size.

\subsubsection{Message Types}

The DMIP protocol is designed to be minimal, to ease development of compatible applications. The message names and their corresponding message type are listed in 
Table 20. A description of each message type, and the message data, if applicable follows the table. 
Table 20: DMIP Message Types

\begin{tabular}{|l|l|l|}
\hline Message Name & Message Type Enumeration & Sent By \\
\hline Acknowledge & 0 & Client, Service \\
\hline Error & 1 & Client, Service \\
\hline Close & 2 & Client, Service \\
\hline Malformed & 3 & Not sent \\
\hline Abilities & 7 & Client \\
\hline RemoveAbilities & 8 & Client \\
\hline AddChannel & 9 & Service \\
\hline RemoveChannel & 10 & Service \\
\hline SetChannelOpt & 11 & Service \\
\hline Data & 24 & Client, Service \\
\hline MultiPayload & 254 & Client, Service \\
\hline SplitPayload & 255 & Not Sent \\
\hline
\end{tabular}

\subsubsection{Acknowledge Message}

Acknowledge messages have two purposes, confirm an AddChannel message (section 5.6.2.7) or sent as a keep-alive message (section 5.5). Acknowledge messages optionally contain a 16-bit unsigned integer, which is representative of the Channel ID added by the client. If the value is omitted, or the Channel ID does not exist, it is to be interpreted as a keep alive only. When confirming an AddChannel message, the Acknowledge message should only be sent when the client is prepared to start receiving data. If the channel is purely used for sending data to the service, then it is not required to be sent as the initial Data message sent by the client is taken as implicit acknowledgement of the AddChannel message. Messages sent by the service endpoint on the channel before acknowledgement are undefined and should not be processed by the client if received, unless these messages are received in a MultiPayload message (section 5.6.2.10) with a matching ChannellD as the AddChannel message.

Table 21 Acknowledge Message Structure

\section{Message Type: Acknowledge (0)}

\begin{tabular}{l|l|l|}
\hline Field & Size & $\begin{array}{l}\text { Description } \\
\text { ChannelID }\end{array}$ \\
\hline 8 bits & $\begin{array}{l}\text { (Optional) } \\
\text { The ID of a channel that is ready to send and/or receive data }\end{array}$ \\
\hline
\end{tabular}




\subsubsection{Error Message}

An Error message is a generic message passing mechanism, it allows passing of a 16-bit Unicode formatted byte string representing an error message. The ChannellD field allows communication of the specific channel generating the error, a value of 0 (zero) indicates an error that is not channel specific. Error messages do not change the state of a DMIP session (terminate a session nor indicate the removal of a channel). However Error messages should be sent by clients when an AddChannel request (section 5.6.2.7) is not fulfilled, and thus can represent the lack of a channel within a session.

Table 22 Error Message Structure

\section{Message Type: Error (1)}

\begin{tabular}{|l|l|l|}
\hline Field & Size & Description \\
\hline ChannelID & 8 bits & The ChannelID of the channel generating the error \\
\hline Message & variable & $\begin{array}{l}\text { A 16-bit UTF string, providing more information about the } \\
\text { error }\end{array}$ \\
\hline
\end{tabular}

\subsubsection{Close Message}

The close message is used to terminate a session, a Unicode byte stream is optionally attached. To provide rationale/reasoning for the connection closing.

Table 23 Close Message Structure

\section{Message Type: Close (2)}

\begin{tabular}{|l|l|l|}
\hline Field & Size & Description \\
\hline Message & variable & $\begin{array}{l}\text { A 16-bit UTF string, providing more information about the } \\
\text { reason the connection was closed }\end{array}$
\end{tabular}

\subsubsection{Malformed Message}

This Message type is reserved for internal use, and allows processing of incomplete or malformed messages on either endpoint. It is not explicitly part of the network protocol, except that the message type value (3) is reserved. 


\subsubsection{Abilities Message}

The Abilities message is sent by a client device, it is the only message that can initiate a clientservice session. The purpose of the Abilities payload is twofold; to specify what kind of layouts the device can support (if any) and which channel types a client can utilize. Subsequent Abilities and RemoveAbilities messages can be sent to add and remove channels should the client's feature list change during an active session.

If an Abilities message is received within an active session (beyond the session initiating Abilities message), it is to be processed cumulatively. These messages will add to the list of session supported Channel Types or update the supported MetaChannels, toggle the IsDiscrete parameter or change the Multiplicity for previous declared Channel Types. Subsequent The Abilities message SHALL NOT trigger the direct removal of channels by the client, if Multiplicity is updated to a value below the current number of active instances of the given Channel Type the service MUST respond by explicitly removing (see RemoveChannel, 5.6.2.8) select channels until the new Multiplicity limit is observed, or discontinue the session. 
Table 24: Abilities Message Structure

Message Type: Abilities (7)

\begin{tabular}{|c|c|c|}
\hline Field & Size & Description \\
\hline NumberOfLayouts & 8 bits & The number of layout options included \\
\hline NumberOfChannels & 16 bits & The number of channels included \\
\hline \multicolumn{3}{|l|}{ Layout Options 0..* } \\
\hline UniqueFeatureID & 8 bits & $\begin{array}{l}\text { A value for layout identification so that multiple displays } \\
\text { can be used }\end{array}$ \\
\hline$X$ & 16 bits & Extent in pixels \\
\hline $\mathrm{Y}$ & 16 bits & Extent in pixels \\
\hline Z & 16 bits & Extent in pixels \\
\hline \multicolumn{3}{|l|}{ Channels 1..* } \\
\hline MetaChannelCount & 8 bits & The number of meta channels $(0-255)$ \\
\hline \multicolumn{3}{|l|}{ Meta Channels O..* } \\
\hline MetaChannelsID & 32-bits & The Channels ID of a meta-channel \\
\hline IsDiscrete & 1 bit & Whether the data is discrete or continuous \\
\hline Reserved & 7 bits & \\
\hline Multiplicity & 16 bits & $\begin{array}{l}0 \text { is any number, otherwise represents how many of these } \\
\text { channels the client supports }\end{array}$ \\
\hline ChannelType & 32 bits & $\begin{array}{l}32 \text { bit standardized ability identifier for the type of data } \\
\text { sent }\end{array}$ \\
\hline
\end{tabular}

\subsubsection{RemoveAbilities Message}

The remove abilities message allows channels to be removed from an active session. Upon receipt of a remove abilities message, the service should stop processing or sending any messages for any channel of the given channel types. The remove abilities message contains a list of 32-bit values representing the channel types that are no longer valid. If the modified list of Channel Types is not supported by the service, it MAY explicitly disconnect the client (see section 5.6.2.3) or it MAY maintain the session, the behaviour is definable by the DMIP service author.

Table 25: Abilities Message Structure

\section{Message Type: RemoveAbilities (8)}

\begin{tabular}{|c|c|c|}
\hline Field & Size & Description \\
\hline ChannelType & 32 bits* $i$ & $\begin{array}{l}32 \text { bit identifiers for the channel types to be invalidated, any } \\
\text { number }(i) \text { of channel types can be specified }\end{array}$ \\
\hline
\end{tabular}




\subsubsection{AddChannel Message}

The AddChannel message is used by the service to request that a connected client add a channel to the active session. An 8-bit layout ID allows a specific layout to be used see Abilities, A 16-bit identifier for the channel is specified, this identifier must be unique for the active session, but can be reused if a RemoveChannel message (section 5.6.2.8) is sent for the identifier prior. The identifier is attached to all related Data messages (section 5.6.2.10) and must be kept and used by both the client and service. The channel type is specified by the ChannelType parameter. The Options parameter carries the payload for the construction of the channel as described in 5.5.1. Table 26: AddChannel Message Structure provides the in-order structure of an AddChannel message.

Table 26: AddChannel Message Structure

\section{Message Type: AddChannel (9)}

\begin{tabular}{|c|c|c|}
\hline Field & Size & Description \\
\hline LayoutID & 8 bits & Specifies which layout the channel should be added to \\
\hline ChannelID & 16 bits & An identifier that the channels instance will be referred to as \\
\hline ChannelType & 32 bits & $\begin{array}{l}\text { The channels feature that is being subscribed/added, initially } \\
\text { specified by the client in Abilities packet }\end{array}$ \\
\hline & Variable & IOption for the Channel Type \\
\hline
\end{tabular}

\subsubsection{RemoveChannel Message}

The RemoveChannel message allows channels to be removed by the service within an active session, this in conjunction with AddChannel allows a dynamic client experience. The RemoveChannel message carries a list of 16 bit ChannellDs, upon sending or receipt or transmittal of a RemoveChannel message, both endpoints should no longer send or process messages for any of the given channels. If an unknown/unused identifier is specified, the identifier should be ignored. After sending a RemoveChannel, request, the ChannelID is then available for re-use for additional channels. 
Table 27: RemoveChannel Message Structure

Message Type: RemoveChannel (10)

$\begin{array}{lll}\text { Field } & \text { Size } & \text { Description } \\ \text { ChannelID } & 16 \text { bits* } i & \text { A list of } i \text { identifiers representing active channels to be removed }\end{array}$

\subsubsection{SetChannelOpt Message}

The SetChannelOpt message, sent by the service, allows options to be set or reset outside of the AddChannel message, more importantly, it is the only means by which to specify metachannel options. The message consists of the 16-bit channel id, the 32-bit channel type and the encoded channel type options payload discussed in 5.5.1. SetChannelOpt messages can be sent by the service for any active (acknowledged) channel, additionally, they are valid as part of a MultiPayload message, provided a prior AddChannel message for that channel is given within the MultiPayload, this allows meta-channels to be initialized more easily and concisely.

Table 28: SetChannelOpt Message Structure

\section{Message Type: SetChannelOpt (11)}

\begin{tabular}{|c|c|c|}
\hline Field & Size & Description \\
\hline ChannellD & 16 bits & The identifier of the channel \\
\hline ChannelType & 32 bits & $\begin{array}{l}\text { The channel's feature that is being subscribed/added, initially } \\
\text { specified by the client in Abilities packet }\end{array}$ \\
\hline Options & Variable & ChannelOption for the Channel Type \\
\hline
\end{tabular}

\subsubsection{Data Message}

Data messages are the main transport mechanism for information within the DMIP protocol. A data message contains the associated Channel ID, generated by the service and included as part of an AddChannel message. The 16-bit channel ID is prepended to a variable length byte stream along with the ChannelType, which can be the type given the specific channel instance or any of its associated MetaChannels, this enables decoded according to the given Channel Type definition 5.5.1. 
Table 29: Data Message Structure

Message Type: Data (24)

\begin{tabular}{lll} 
Field & Size & Description \\
\hline ChannellD & 16 bits & An identifier for the channel instance that the data is from \\
\hline ChannelType & 32 bits & The ChannelType of the data \\
\hline Data & Variable & Data in the specified ChannelType format
\end{tabular}

\subsubsection{MultiPayload Message}

MultiPayload messages can carry multiple payloads. This allows lower network overhead when dealing with smaller payloads. It also eases the development use and initialization of metachannels. Each encapsulated message is formatted with an 8-bit message type followed by a 16-bit unsigned integer length of the payload, and the byte encoding of the payload. The order of payloads within the MultiPayload message must be observed by network endpoints and should be processed synchronously (the first payload must complete processing before the next is processed).

Table 30: MultiPayload Message Structure

\section{Message Type: MultiPayload (254)}

\begin{tabular}{|c|c|c|}
\hline Field & Size & Description \\
\hline \multicolumn{3}{|l|}{ 1..* } \\
\hline MessageType & 8-bits & The MessageType of the proceeding payload \\
\hline Length & 16-bits & The octet length of the proceeding payload \\
\hline Payload & $\begin{array}{l}\text { Length } \\
\text { octets }\end{array}$ & The payload of the encapsulated message \\
\hline
\end{tabular}

\subsubsection{SplitPayload Message}

A SplitPayload Message is a message that has been split into 2 or more messages to overcome the header length field limitation (16-bit) and to support potential limits in frame sizes over the transport layer protocol. The SplitPayload MessageType in not directly transported over the network, instead the 16-bit DMIP header fields PacketCount and PacketNumber are used. This allows a maximum message size of approximately 4 gigaoctets. The SplitPayload MessageType 
enumeration value (255) is reserved to allow the SplitPayload MessageType to be more simply represented in software implementations of the protocol.

\subsection{Field Sizes}

The design of a novel protocol requires estimation of a number of parameters, including the size of fields and types of messages included. Every effort has been made to avoid choosing values arbitrarily and to allow the representation of all imaginable types of data. Estimates were aimed at keeping message header and overhead low as well as keeping data byte-aligned. The result is that there are limits to several values within the protocol. Notably, there is a limit of $2^{32}$ different Channel Types that a client/service can understand, there is a limit of $2^{16}$ active Channels in a single DMIP session and a single DMIP message has a maximum size of 64 kilobytes. Maximum message size of 64 kilobytes combined with the $2^{16}$ maximum number messages within a single payload (Section 5.6.2.12 SplitPayload Message), means that a single payload has maximum size of 4 gigabytes. Data types that are envisioned to potentially exceed this limit MUST incorporate splitting/recombining within the Channel Type definition.

\subsection{UDP Stream Data}

The User Datagram Protocol allows lower latency [2], and MAY be used as a transport mechanism for some Channels. DMIP services SHOULD have the ability to utilize UDP to send or receive Data messages, but MUST only do so for a channel type whose abilities, specified by the client include an IsDiscrete (Abilities Message, 5.6.2.5) property set to true (1).

\subsection{Layouts}

Layouts represent combination of channels on a client device. Allowing the specification of a layout allows host devices to control how multimodal interaction channels will be displayed a client device. In the current version of the protocol, only vector layouts are supported through the use of the Positionable3D meta-channel. However, extension of the protocol could provide meta-channels to support additional types of layout and positioning. 


\subsection{Timeouts}

DMIP connections are considered terminated after 10 seconds $(10,000$ milliseconds) of inactivity. Inactivity means no receipt of a message. Both clients and services should observe this restriction. Sessions terminated due to timeout should send a close message (section 5.6.2.3) to the remote endpoint if the TCP socket is still available. Both endpoints should ensure that a message is sent at least every 7 seconds, making use of the Acknowledge message (section 5.6.2.1) as a keep alive message if necessary. 


\section{Protocol Flow}

In order to describe the intended flow of DMIP sessions using the Protocol (section 5), this section provides an overview of DMIP session flow (section 6.1). Additionally, a number of examples of typical sessions are provided (section 6.2).

\subsection{DMIP Session Overview}

A DMIP session is a 1 to 1 , single TCP endpoint to TCP connection, which can be supplemented by UDP, all session management messages are, however sent using TCP to ensure, in order delivery [2]. Sessions are initiated by clients (section 6.1.1), if services accept the clients, they can add channels (section 6.1.2) to communicate (section 6.1.3), active sessions can have any number of channels added to them, and they can be removed (section 6.1.5) allowing dynamic user interfaces and other scenarios (section 6.1.4). Session are terminated by either endpoint explicitly or implicitly after a timeout (section 6.1.6).

\subsubsection{Session Initiation}

A session is initiated from a client by sending an Abilities message (section 5.6.2.5), providing a listing of Channel Types (section 5.5) it can support, Meta-channels (section 5.5.5) those channels can support, as well as information about display devices available for Positionable3D Channels (section 5.5.5.2). This information should be stored by the service so that it can evaluate compatibility, and respond appropriately to the client during the session's lifecycle.

Service applications can explicitly reject clients by sending a Close message (section 5.6.2.3) in response to an initial Abilities message (section 5.6.2.5), this allows an explanation for connection refusal to be provided. The service can reject a client for any reason. Wherever possible, services should provide rationale for rejection, to enable user understanding [28]. Examples of reasons for rejection are; incompatible/insufficient Channel Types available on the client and service capacity/user limits reached. Rejection is implied if the client does not receive a response from the service within the timeout period (section 5.5). The rationale for this is to handle situations where the service is unavailable/unable to respond, but should not be 
purposely used by service authors, as doing so inhibits user understanding because it limits visibility of system status [28].

\subsubsection{Channel Initiation}

If the service accepts the client, it can add compatible channels, using AddChannel (section 5.6.2.7) messages. A service should not send messages for a channel until either an initial Data (section 5.6.2.10) or Acknowledge (section 5.6.2.1) message is received from the client for the channel. If the client is unable to add a channel, it should send an Error message (section 5.6.2.2) containing the channel's ChannellD, to the service.

Meta-channels provide augmentation of channels by allowing additional data types to be specified for a channel (section 5.5.5). Meta-channels (as specified by the client during session initiation, 6.1.1) for a channel are automatically available when it is added, default options are however, undefined. Initial options for Meta-channels can be bundled in a MultiPayload message (section 5.6.2.11) as SetChannelOpt messages (section 5.6.2.9) by the service if initial values are required.

These channels are the method in which data is communicated within DMIP, the primary identifier for a Channel is its ChannelID, this need to be registered by, and handled appropriately at both endpoints.

\subsubsection{Active Channels}

Once a channel is added and acknowledged, the endpoints can communicate using Data messages (section 5.6.2.10) in the format of the Channels Type's associated data type, described in 5.5.3. Meta-channel data is communicated using the same ChannellD as the attached channel, with the Meta-Channel's type as the channel type (allowing for only one instance of each meta-channel per channel). Additionally options for the channel and associated meta-channels can be updated by the service using SetChannelOpt messages. When a complete Data or SetChannelOpt payload is received at an endpoint, it should be decoded and processed to generate relevant actions. 


\subsubsection{Active Session}

Within an initiated session, all valid DMIP messages are processed. Messages that refer to an inactive channel are typically ignored. A client can modify its available channel type abilities by sending Abilities or RemoveAbilities messages (section 5.6.2.8, 5.6.2.8). If a client removes a channel type ability that is currently in use within the session the service should discontinue its use, terminating the session with a Close message (section 5.6.2.3) if required. Channels removed in this way are then considered inactive, and may require removal within the client's interface. Services may request the addition or removal of channels to the session at any time through the use of AddChannel and RemoveChannel request, as described in 6.1.2 and 6.1.5 respectively. By removing and adding channels, services can generate dynamic applications that can efficiently make use of the remote client's abilities.

\subsubsection{Channel Termination}

Active channels can be terminated explicitly by way of a RemoveChannel request from the service. When the service sends this request, it is indicating that it is not going to send or process incoming additional messages referring to the channel. When the client receives a RemoveChannel request, it stops initiating actions for that channel; Data messages are no longer sent and any visual representations of the channel in the client interface are removed.

Channels can be implicitly removed through a RemoveAbilities request from the client, in which case the channel should be explicitly removed from the client so that the ChannellD can be reused and any interface elements relating to the channel are removed.

All active channels are also terminated when a session is terminated, and both endpoints can safely perform removal and cleanup of any channels associated with the session.

\subsubsection{Session Termination}

Session can be terminated by either the client or the service within an active session by sending a Close request. The Close should be the last message transmitted for any session. When an endpoint receives a Close request it terminates the session and removes any resources 
associated with the session. If a client receives a Close request, the message, within the device's interface should be communicated to the end user. The service may log the session, but should not inhibit other users from connecting, initiating sessions, or using the service's functionality.

Sessions can also end implicitly if the session is inactive for a duration that exceeds the timeout length (section 5.5). Both endpoints follow the same steps as above, however both endpoints can use a generic timeout message to show the user, or log, in absence of a Close Message.

\subsection{Examples of DMIP Message Passing}

Explanations and supporting diagrams demonstrating several DMIP session scenarios are presented within this section, the rationale being that they provide specific visual examples to aid in conveying DMIP usage scenarios. The examples provided assume context, meaning that it is assumed that a DMIP client and service exist, and the client has the IP endpoint for the service.

\subsubsection{Client Request Session}

After or asynchronous to a client acquiring a service endpoint, it must;

1. Initialize and enumerate its DMIP abilities.

- Channel types it can support.

- Layout format(s) and properties.

2. Create a DMIP Abilities (section 5.6.2.5) message.

3. Send the message over a TCP connection to the service's IP endpoint.

4. Listen for and respond appropriately to messages.

- With the same port and address the abilities message was sent from.

- TCP must be listening, UDP is optional.

The service endpoint then takes action determining if the client's Abilities are compatible and can: 
5. If it is not able to communicate with the client:

- Ignore the request.

- Preferably send an explicit close message (section 5.6.2.3) back to the client.

6. If it can interact with the client, a session in the service process is created.

7. Acknowledge (section 5.6.2.1) or AddChannel (section 5.6.2.7) message(s) are sent to the originating endpoint of the Abilities message.

Figure 4: Session Request and Initiation, below shows a diagram that represents the intended flow of this process.

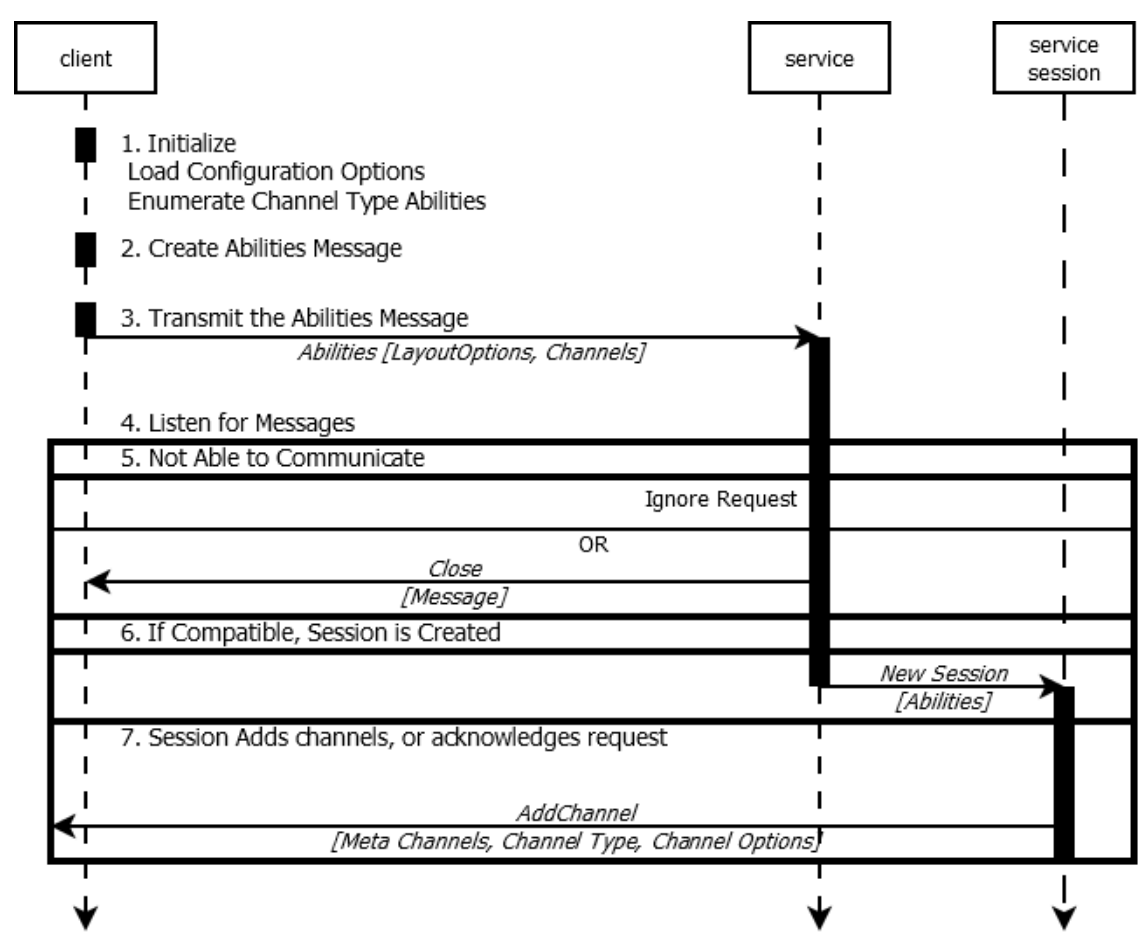

Figure 4: Session Request and Initiation

\subsubsection{Adding Channels and Meta-Channels}

DMIP services drive the communication channels that can be used within a DMIP client-service session. Channels are added only by the service, using the AddChannel message (section 5.6.2.7). Meta-channels that a client can use for a channel type are included in Abilities messages, the service should retain this information if it is to use those meta-channels. The AddChannel message specifies that the given channel type instance's channel options. However 
any attached meta-channels have default, undefined initial values set for options. Options for meta-channels are set using SetChannelOpt messages (section 5.6.2.9), these messages can be sent for a channel after it is confirmed by the client, or in a MultiPayload message (section 5.6.2.11), provided it is in the same MultiPayload message and proceeds the AddChannel payload for the attached channel.

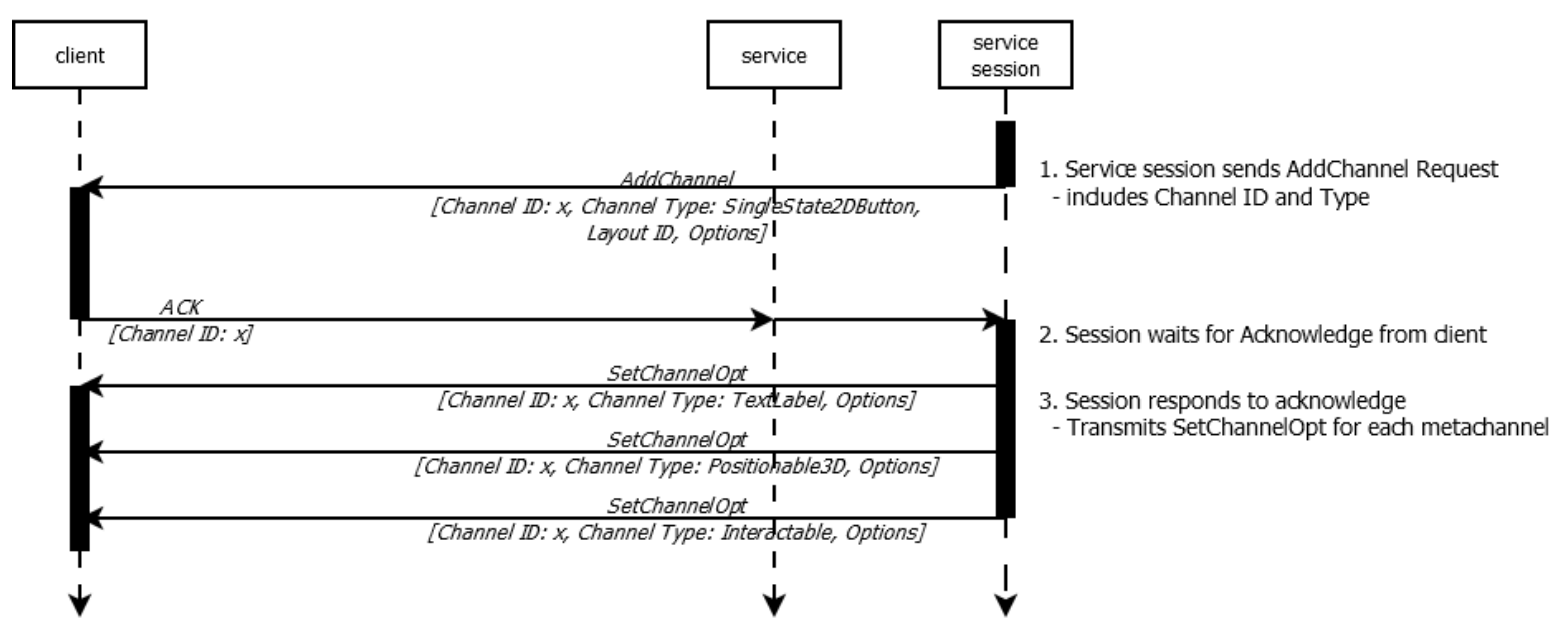

Figure 5: Distinct Meta Channel Setup

Figure 5: Distinct Meta Channel Setup shows DMIP steps used to configure meta-channels associated with a channel distinct from an AddChannel message, note that the method of attachment is matching Channel ID values ( $x$ in the diagram). This sample flow highlights the fact that meta-channel options can be set at any time after a channel has been confirmed. The rationale for choosing this method is that it allows properties to be calculated and asynchronously set. Additionally, if meta-channel options are to be set programmatically as a session progresses, it may not serve any purpose to set these options immediately. 


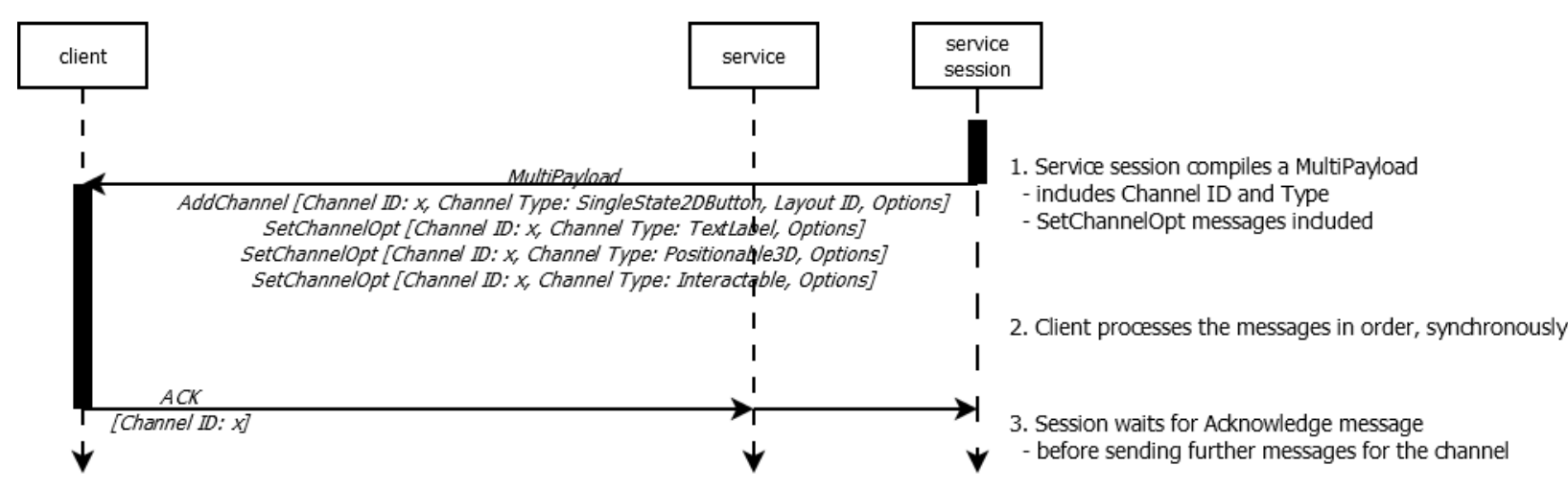

Figure 6: Inclusive Meta Channel Setup

Figure 6: Inclusive Meta Channel Setup shows the networking steps for adding a channel and the same associated meta-channels is in the previous example (Figure 5). As dictated by the protocol specification MultiPayload messages (section 5.6.2.11) must be processed in order, thus ensuring that the subsequent processing of the SetChannelOpt have a valid target. Specifying meta-channel options using this method provides a reduction in network overhead, and reduces logic required for setting up channels from the service (no need to wait for Acknowledge to send initial options). And allows design tools to be more simply implemented. The XML layout language used in the evaluation implementation (section 7.2.6.1) uses this method.

\subsubsection{Sample Session, Button Based Menu}

Most applications will not make use of the same modes throughout the user's usage of the application. An initial menu, prompting the user for input, to choose various areas of application functionality is an often used example of this. In the PowerPoint Controller Service, discussed in section 7.2.5.4 a simple menu is provided if the service is running but no show is running. As seen in Figure 7: Simple User Menu, this menu allows the user to request that the show be started or allows to the user to explicitly end the session. This is accomplished using an instance of the SingleState2DButton for each menu option. Once the user makes the "Start Show" selection, the service no longer makes use of the channels associated with the buttons, 
and removes them to re-use screen real estate and reduce session overhead for managing channels.

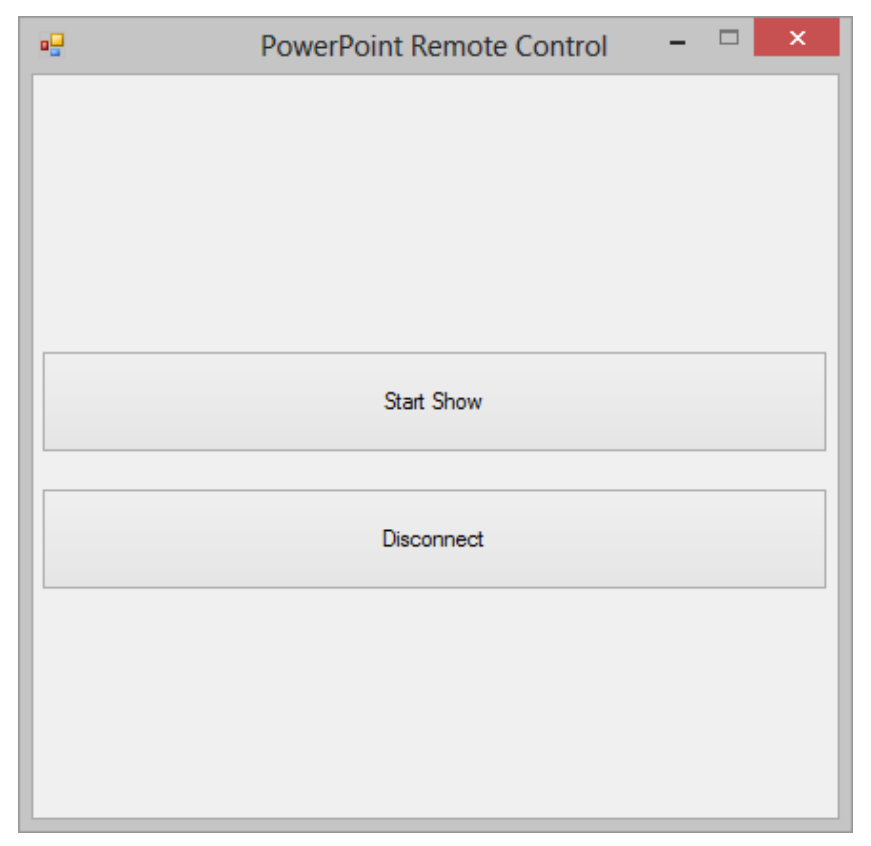

Figure 7: Simple User Menu

After the RemoveChannel requests have sent to the client, an interface for interacting with the active show are provided to the user, as seen in Figure 14: Windows Forms Client. In this way interaction elements can be added and removed to provide dynamic applications. Figure 8: PowerPoint Controller Menu to Show Layouts, shows a simplified network diagram of the DMIP messages used in the PowerPoint Controller Service when switching to the show running interface from the show menu interface. 


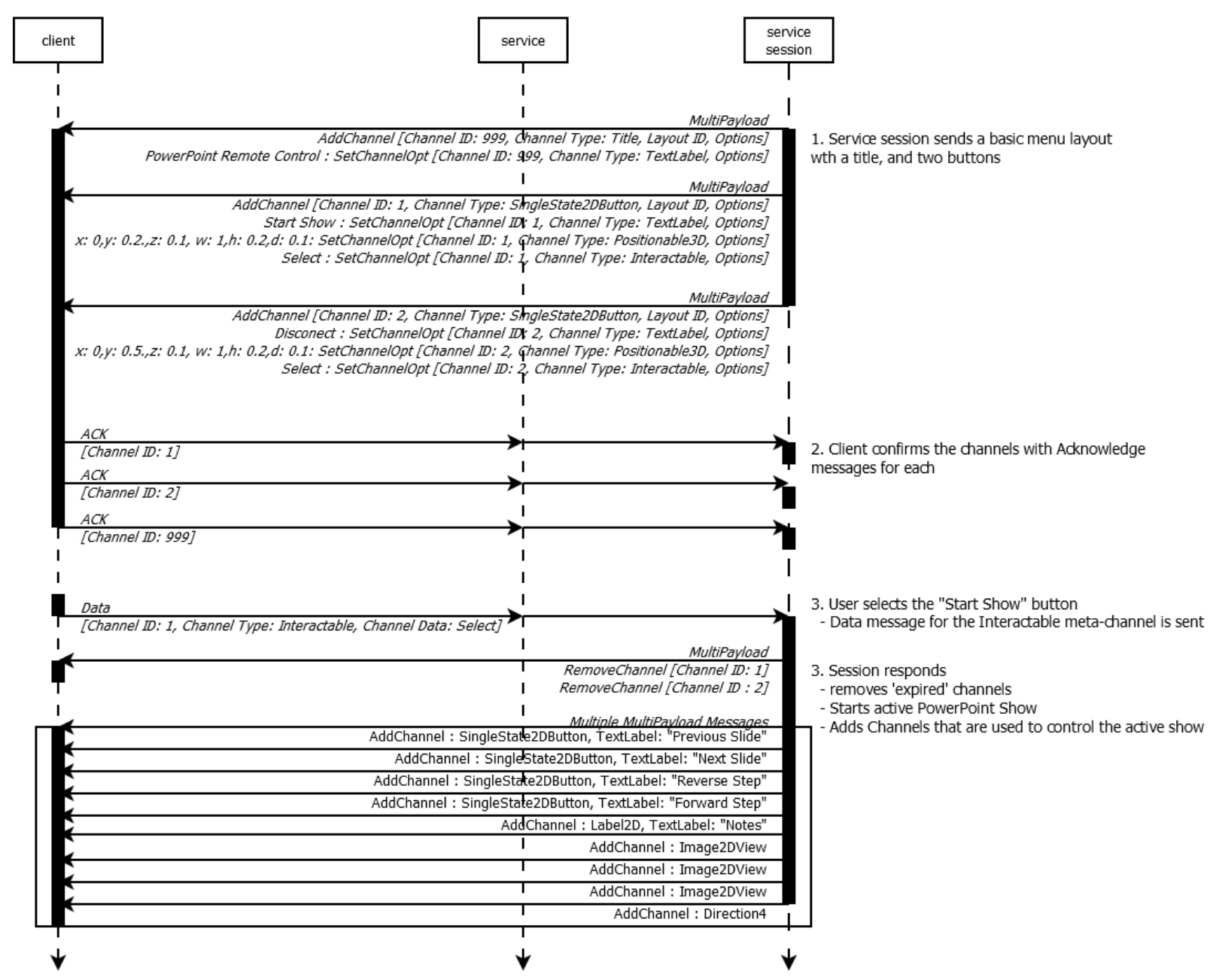

Figure 8: PowerPoint Controller Menu to Show Layouts

\subsubsection{Client Remove Abilities}

Only DMIP services have the ability to explicitly add remove channels, that is, AddChannel

(section 5.6.2.7) and RemoveChannel (section 5.6.2.8) messages can only be sent from the service endpoint. The client endpoint can however modify the list of abilities it supports. When a DMIP client connects to a service, it sends a list of abilities, via an Abilities message, this list can be modified by the client with subsequent Abilities and RemoveAbilities messages (section 5.6.2.5, and 5.6.2.6). If a RemoveAbilities is received by the service that contains a Channel Type that is needed for the session to continue, then the session can either be terminated or can be retained. A service might retain a session that cannot progress so that should client functionality return, the application state could be maintained. There are a number of alternate 
methods of retaining session state, and in most cases the service will explicitly end the session.

An network example of an explicit close in response to a RemoveAbilities, is seen in Figure 9:

Client Configuration Change.

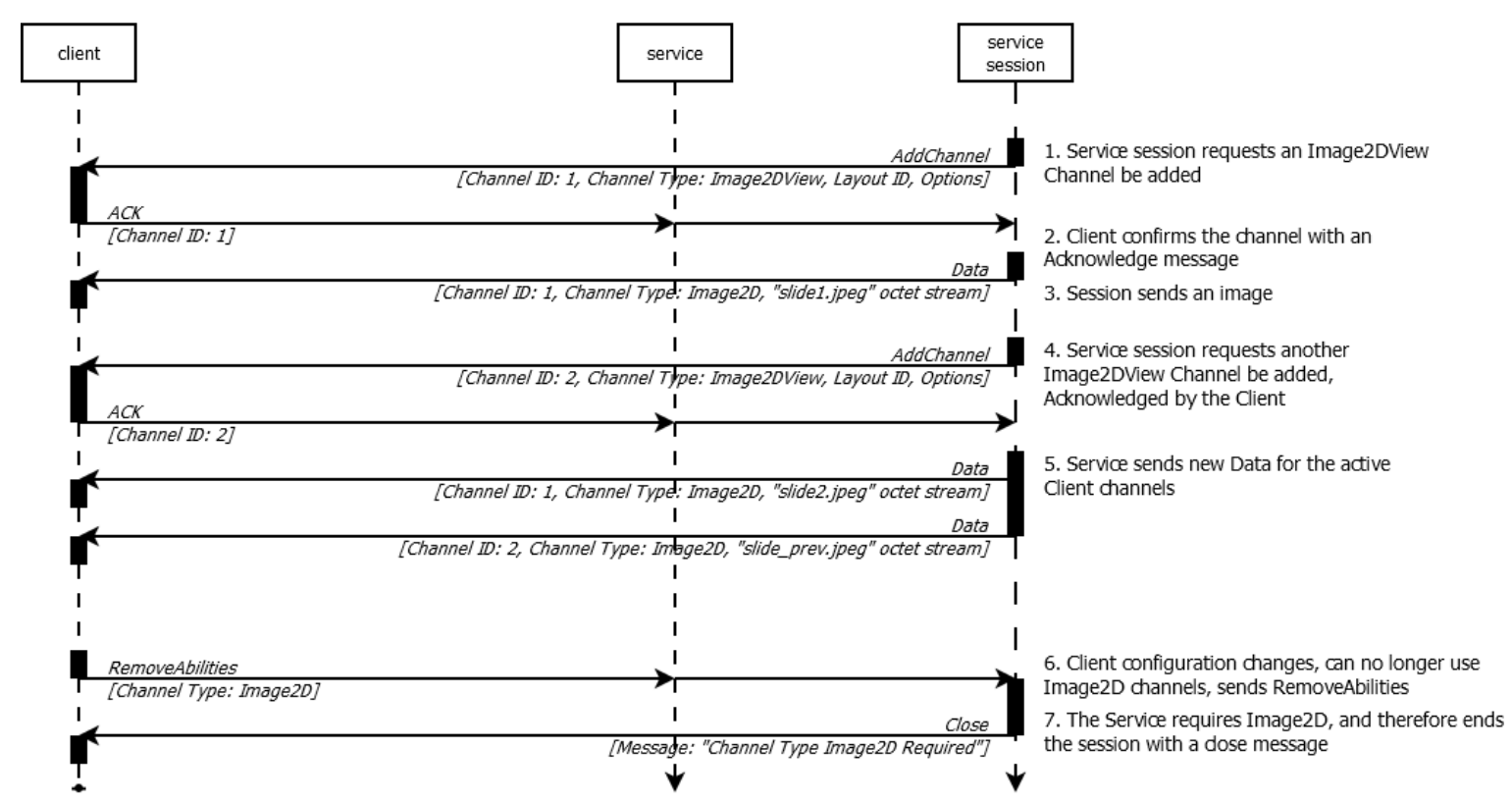

Figure 9: Client Configuration Change 


\section{Evaluation}

To validate that DMIP is able to meet the goals set out in (section 1), a review of potential data types was performed further described in (section 7.1). To validate whether DMIP is functional and can be used to meet the stated requirements, it was implemented on 2 platforms as discussed in (section 7.2).

\subsection{Validity of Channel Types System}

Before the design process began it was thought that a system that could natively support any interaction mode type could be designed. However it was noted that the resultant data transferred would be too varied and it would not be possible to define what modes/abilities a session could use and the data transferred would not contain enough intrinsic meaning to be directly consumable. Therefore, this view was modified as development of the protocol progressed and was replaced with the concept of providing an extensible framework that defines strictly defined Channel Types and allows development of further Channel Types outside the explicit protocol. This raised the concern that $3^{\text {rd }}$ party developers may incorporate proprietary Channel Types, limiting the cross compatibility of clients, counter to the goals of this work. It is therefore our intention that extension Channel Types should be catalogued and reviewed for standardization as part of future work.

Considering that development of additional standard Channel Types requiring minimal effort and with inclusion of extensible Channel Types we aim to allow an expanding list of interaction types to be supported by clients and services. To verify that all imaginable interaction types could potentially be supported, a review of potential raw data types, common UI controls and interaction modes defined by Bersen [29] was performed, as well as an informal web survey of users, asking how they interacted with everyday objects.

\subsubsection{Common UI Controls}

Common user interface controls were observed by looking at user interface implementation tools, namely Microsoft Visual Studio 2012 Windows Forms designer and the Android Layout 
Editor plug-in for Eclipse. Rigorous evaluation was not performed as the controls used for these platforms form the basis of how the protocol was designed. In these systems, various properties for a control can be configured, this can be mapped directly onto Channel Type Options (section 5.5.2). Common properties (those shared amongst most types of control and across platforms) can be mapped to meta-channels (section 5.5.5). Controls typically generate events indicating some status has changed or new data has arrived, these are often generated by the user interacting with the control. Events are mapped to Channel Type Data (section 5.5.3) and while a single control might provide a variety of events, the design of Channel Type Data types is flexible and thus could allow for any combination or digital events to be represented.

\subsubsection{Raw Data Types}

Basic digital-format data types for example images, video, sound, and documents, are represented by octet streams or blocks of binary encoded data and be simply transported using Channel Type Data, and can be split up, or transmitted using UDP datagrams for timely data. Raw files could be transferred in this way. Analog data sources could also be transmitted if they are first converted to digital. This conversion has been shown to be possible for many kinds of data, the discussion of which is beyond the scope of this thesis.

\subsubsection{Bernsen's Multimodal Interface Modes}

Using Niels Ole Bersen's generic taxonomy of unimodal modalities [29] as a guide provided a comprehensive listing of potential data types to consider. These were reviewed and were found to be directly translatable to digital raw data types or subject to analogue to digital signal processing, which while potentially complicated, is required in order to capture/process that data on the digital devices used by DMIP, and is therefore not actionable consideration for the design of the protocol. 


\subsubsection{Interaction Ledger}

An informal study, asking users to specify interactions with everyday objects around them. The survey and results can be seen in Interaction Ledger. While this survey was very open-ended it provided insights as to how users interacted with the real world objects around them. These interactions can all be broken down into one or more modalities in defined by Bernsen in his taxonomy [29], and are therefore possible to transport using the DMIP's extension channels (section 5.5.6) or standardized Channel Types in future revisions.

Further, the individual tasks where codified to help identify the types of general interaction steps taken by the user to accomplish regular/common tasks they encountered in their daily lives. The actions taken were classified as either Motion based, Selection based or both. A chart representing the results of this process is below, Figure 10: Interaction Ledger Results.

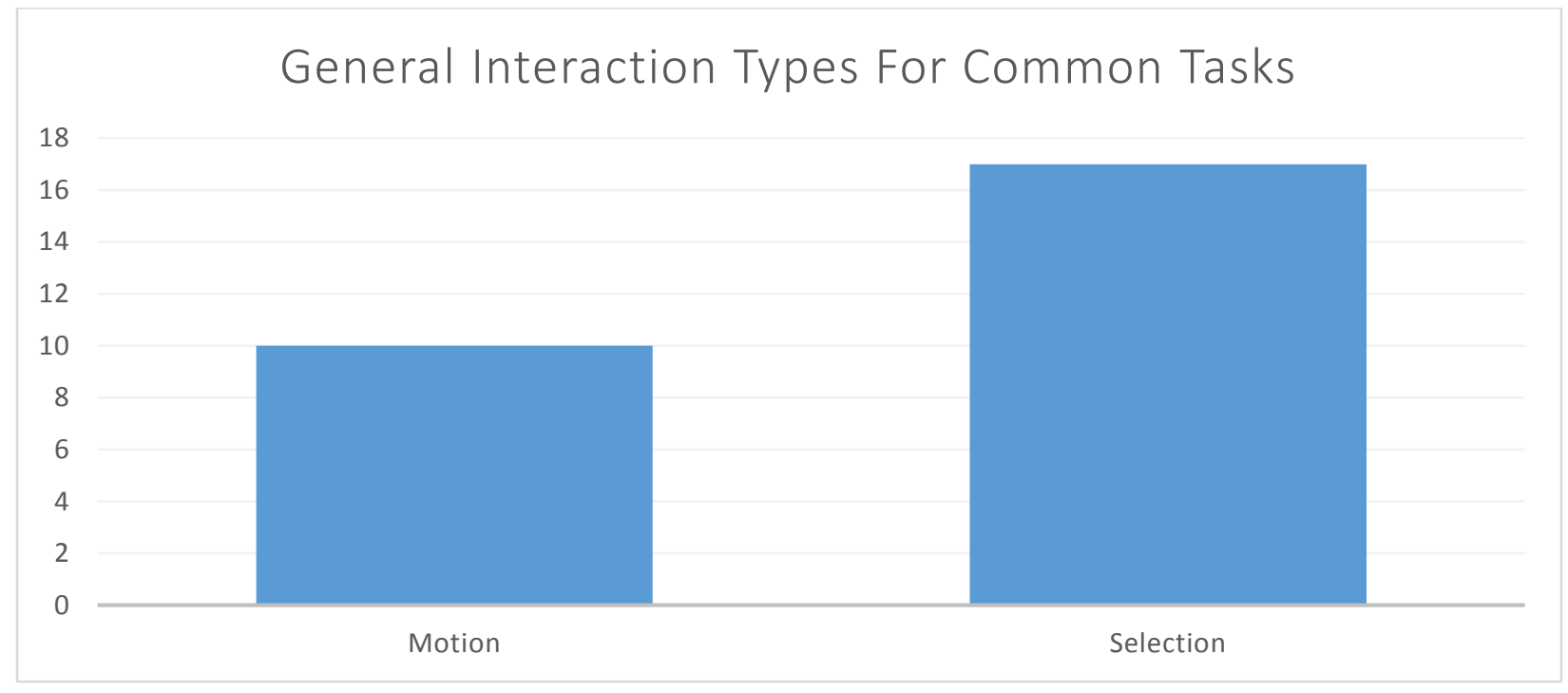

Figure 10: Interaction Ledger Results

Out of the 20 entries, a large number of the specified tasks (85\%) included selection subtasks, and thus the Channel Types created for the initial version of the DMIP protocol were focused on selection, buttons, labels, range selects, list boxes and character channels were implemented to support selection tasks. While a significant number $(50 \%)$ of subtasks incorporated motion, only the $3 \mathrm{D}$ and $2 \mathrm{D}$ motion channels were introduced, the rationale for this is that the interpretation 
of motion signals (or gestures) is beyond the scope of this work, but can be supported by these two channels. The responses and the respective codification can be seen in Appendix A: Interaction Ledger.

\subsection{Protocol Implementations}

Initially, an object oriented software design was created to fulfill the requirements set out by the protocol. The design of the implementation application is focused on a central package, called Protocol which encompasses all low level functionality for both clients and services. It provides the transport functionality, message processing, channel type definitions, and event dispatch as well as managing queues to support SplitPayload (section 5.6.2.12) and MultiPayload (section 5.6.2.11) messages. Centralizing the common functionality reduces the amount of effort required to implement the combination of clients and services, particularly when they share the same platform. This benefit is generated by eliminating code duplication, reducing the development time, errors and maintenance effort. Section 7.2.1 outlines the pieces of the protocol package.

\subsubsection{The Protocol Package}

For the evaluation Implementation, a shared package that provides common DMIP functionality for both clients and services was created. Both client and service APIs rely directly upon this, the protocol package. A representation of the dependency is seen in Figure 11: Shared Protocol Package. 


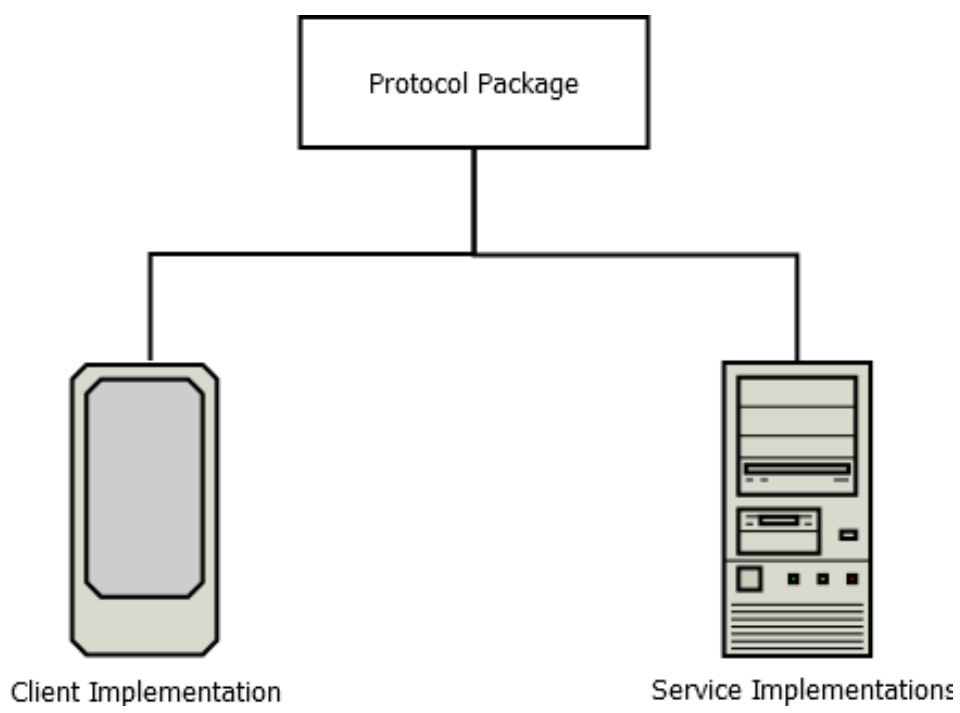

Figure 11: Shared Protocol Package

A diagram identifying the main pieces of the protocol package is provided as Figure 12:

Implementation Protocol Package. Description of these pieces and of how they are utilized to implement the DMIP specification follows. Instances of the Connection class (section 7.2.2) manage single DMIP sessions, which is used to transport MessageType (section 7.2.4) messages, which can contain ChannelType (section 5.5.3) data.

\section{Protocol Package}

\begin{tabular}{|c|}
\hline Connection Class \\
\hline SplitPackets Hash Map \\
SendMessage Methods \\
MessageRecieved Event \\
\hline
\end{tabular}

IByteSerializable
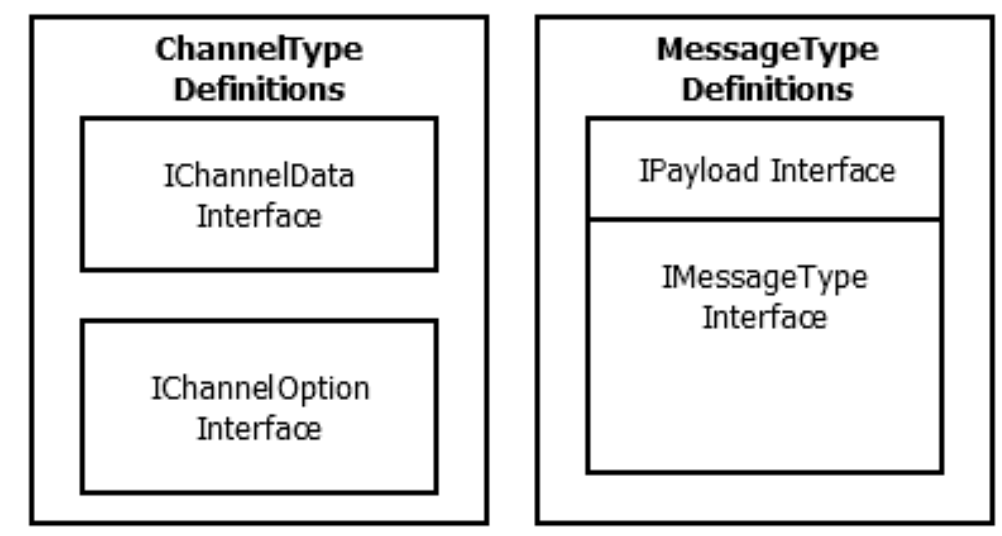

Figure 12: Implementation Protocol Package 


\subsubsection{Connection Class}

The connection class is designed to handle the interaction with lower level operating system networking facilities. Because the operating systems used for implementation project included implementations of both TCP and UDP transport layer protocols, the connection class is used to manage these lower level implementations and provide specific functionality in support DMIP. A connection instance (object) manages a single active TCP connection and optionally a UDP connection, representing a local endpoint and a remote endpoint.

The Connection class is the central component of the demonstration implementation. To achieve the ability to transport DMIP messages, the octet representations of all network transportable data must implement methods to serialize and de-serialize data to/from the formats defined within the protocol (section 5.5) the IByteSerializable interface explicitly identifies the octet encoding and decoding requirement by specifying that methods used for these purposes are included in all types representing data transportable by the connection class. The Connection object is responsible for using these methods to translate messages for transport, or consumption within the DMIP endpoint applications as required. The connection object must utilize separate threads or lightweight processes for each transport layer connection UDP/TCP that is open, to wait for incoming data. An additional thread is additionally required to manage the listening threads, process incoming messages and send outgoing messages. The listen threads should block and consume as few resources as possible when no data is available from the lower level transport layers.

The connection object is created with an initiated TCP/IP endpoint and whether the endpoint is UDP capable. The Connection object is then responsible for both reading and writing data to the transport stream(s) on the given port using network transported byte streams. To encode and decode messages, the Connection object processes external objects that implement the IByteSerializable interface, to ensure that they can be encoded. 
Section 7.2.2.1, Handling Multi-Payload and Split Payload Messages, further describes the special messages that support that enable large payloads (Split Payload) to be sent and small payloads to be combined (multi-payload). Section 7.2.2.2 Octet Encoding (IByteSerializable Interface) and subsequent sections describe the way TCP/IP stream data is read and written using the Connect, Message and Payload Objects.

\subsubsection{Handling Multi-Payload and Split Payload Messages}

The DMIP protocol allows for multi message payloads and multi-payload messages, as such the Connection object must collect and transmit messages that are part of a multi-message payload, and be able to split large payloads.

When a connection object receives a multi-payload message, it is processed as any other DMIP payload, however instead of providing notification of an incoming message (MessageReceived Event, Figure 12: Implementation Protocol Package), notifications for each contained payload are generated, with the order within the MultiPayload message maintained.

Splitting payloads in the implementation(s) is utilized to allow large payloads exceeding the DMIP header length ( 65535 bytes) value to be transmitted, splitting is translucent to DMIP implementation endpoints. Meaning that this process is automatic, but potentially observable. Providing notification of arrival of pieces of a SplitPayload is not currently part of the implementation but is recommended for future work (8.3.2 Additional Implementation Features).

\subsubsection{Octet Encoding (IByteSerializable Interface)}

DMIP specifies 12 formatted message types (section 5.6.2) and an ever expanding list of channel types (section 5.5) that can be transported using the protocol. To be enable conversion between stream compatible octets and code-useable data objects, all transportable types implement the IByteSerializable interface. The interface indicates that methods to perform serialization and de-serialization of encapsulated data must be provided. Additionally, these 
classes all provide a construction method (constructors) to utilize the de-serialization method to initialize an object from its octet-serialized representation, but this requirement is not enforced by the interface specification as it is not possible to define required constructors from interface specifications on the implementation platforms, however this specification is included and must be observed for the design to be functional.

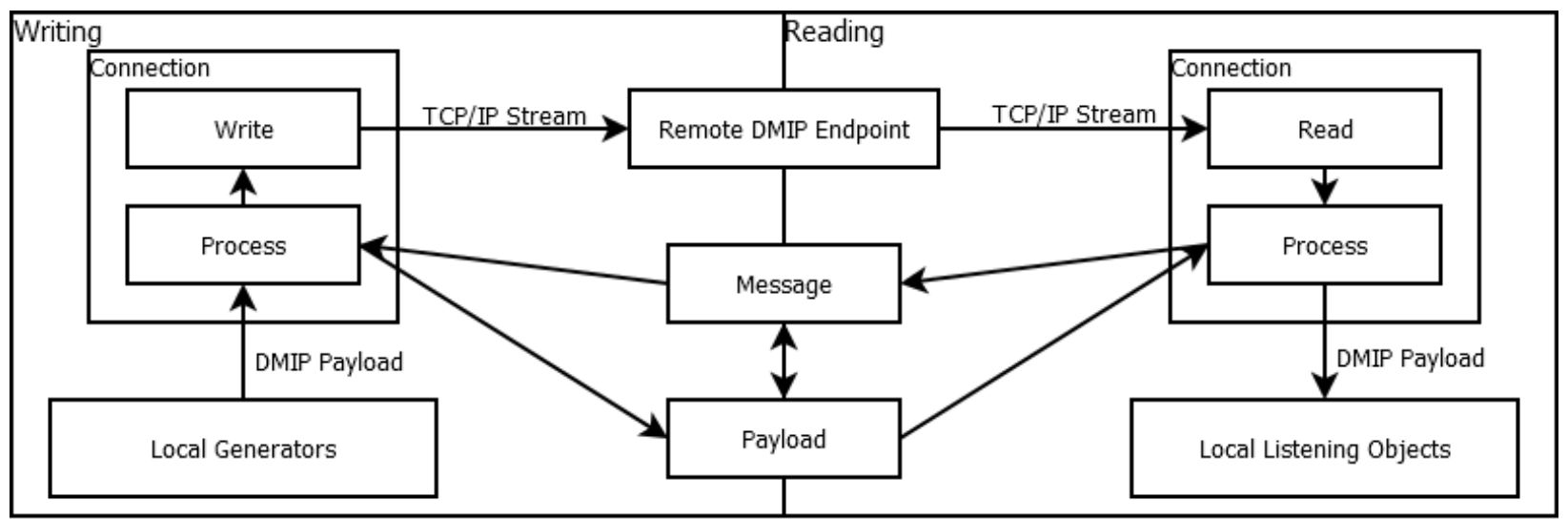

Figure 13: Reading and Writing DMIP Payloads

\subsection{Incoming Data (Reading)}

In order to support the protocol requirements, the Connection object waits and reads all incoming packets on the given transport (TCP and optionally UDP) stream(s), until the connection is terminated. The connection object takes read bytes and processes them into payloads by way of messages (5.6 Messages). Within the implementation, when messages are received, events are fired and listening objects are notified. Pulling the messages from a transport layer protocol and generating actionable DMIP payloads is identical for clients and services as the protocol is designed to have identical message structures for both endpoints, the implementation reflects this by having a single Connection implementation that is shared between DMIP clients and services. Payloads are further decoded and processed using the specific channel type implementation objects. Incoming TCP data is placed in a first in, first out (FIFO) queue so that data can be segmented into Messages as TCP provides data in a 
continuous stream. Messages are either processed immediately as they arrive, or in the case of SplitPayload messages, they are placed in a data object used to collect all the fragments before a message received event is fired.

\subsection{Outgoing Data (Writing)}

The connection object is responsible for taking DMIP messages and sending them over a specified transport layer streams. The Connection object manages the TCP and UDP endpoints, and therefore also tasked with sending UDP targeted messages over TCP if a UDP connection is not available. To accomplish sending messages over the TCP/IP transport layer, the payloads are serialized into octet arrays. Additionally, the Connection object is also responsible for splitting payloads into multiple messages (SplitPayload messages) if they exceed the 16-bit byte size specified in the header (section 5.6.1).

\subsubsection{ChannelType Definitions}

As specified in section 5.5, Channel Types, channel types are comprised of two pieces, Options and Data. These pieces are specified by implementing the IChannelOption and IChanneIData interfaces to create classes for each Channel Type implemented on the platform. The interfaces are architecturally identical, but semantically distinct, so that there is a clear separation in the implementation code. These interfaces require that implementing types have a Channel Type specified.

\subsubsection{MessageType Definitions}

DMIP Payloads and Messages are similar, and for most purposes are the same objects and are implemented using the same classes. These classes all implement the IPayload interface which specifies that member classes must provide byte serialization methods and be able to return one of the 12 message types (section 5.6.2). The IPayload interface is used for network transmitted Messages, Payloads that potentially need to be further aggregated after being split into SplitPayload messages (resulting in single IPayload objects) and payloads containing multiple (MultiPayload messages) messages to be processed in sequence (MultiPayload objects 
can contain multiple IPayload objects). Each class implementing the IPayload interface provides a directly useable representation of the carried data, methods to construct objects of that type from either a stream of octets, or as explicit parameters and methods to serialize the contained data according to the message type's specification within the protocol 5.6.2.

The IMessageType interface is used because IPayload objects must provide both a message type and implement the IByteSerializable interface, and thus IPayload amalgamates the requirements by way of the IMessageType interface.

\subsubsection{Implementation Instances}

Implementation of the protocol was performed on two platforms. The .NET framework was chosen for the initial work, the rationale was the researcher's familiarity with the platform and the broad range of target-able services and platforms. The .NET framework is memory managed development platform that is available on a broad range of devices and allowed rapid development of prototypes ${ }^{1}$. A full implementation of both DMIP client and service endpoints were implemented in .NET, in C\#, the source code, is available from the DMIP website (http://iv.csit.carleton.ca/ dmip/). To test and demonstrate the viability on alternative platforms, the Connection object, all messages coding objects and the client-side application were additionally implemented in Java for Android. The Android implementation is also available from the DMIP website (http://iv.csit.carleton.ca/ dmip/). The .NET implementation is the primary platform for the implementations, the Android implementation is ported from the .NET implementation. Both implementations are released with a permissible MIT license.

The primary purpose of creating the implementation was so that the protocol itself could be verified, to see if it could be utilized to meet the goals set out for it (section 1). Additionally creating implementations in conjunction with the authoring of the protocol in an informal agile development process allowed for issues with the protocol to be identified and adjusted as

\footnotetext{
${ }^{1}$ http://www.microsoft.com/net
} 
authoring and development iterations progressed. Beyond validation, the test implementation allowed for use of the protocol, and helped identify key areas that additional work could be performed to further ameliorate developer, designer and end-user experiences. Further, these implementations provide a reference that can be used to implement and augment services, clients, or as examples to help others port these layers to other platforms.

The Connection class is used as a parent class to Client and Service classes. These classes are designed to augment the Connection class and provide specific features for Client and Service endpoints. Service specific functionality was not ported to Android.

\subsubsection{Implemented Clients}

The Client class is a minimal layer intended to fully implement DMIP client connections, the client layer creates and manages a single instance of the Connection object by providing a service endpoint. A client application, based on this client layer was created, the application provides the Abilities of the client device, initiates the connection, transmits data using SendMessage and responds to incoming data by way of events fired from the Connection object. The client applications implement a number of the DMIP standard channels based on the target platform's capabilities. The channels implemented in each of the two platform's client applications are listed below, in Table 31. Implemented DMIP Channel Types.

Table 31. Implemented DMIP Channel Types

\begin{tabular}{|c|c|c|}
\hline & .NET & Android \\
\hline TextLabel & $\nabla$ & $\nabla$ \\
\hline Positionable3D & $\nabla$ & $\nabla$ \\
\hline Interactable & $\nabla$ & $\nabla$ \\
\hline Enabled & $\nabla$ & $\nabla$ \\
\hline SingleState2DButton & $\nabla$ & $\nabla$ \\
\hline Label2D & $\nabla$ & $\nabla$ \\
\hline RangelD & $\nabla$ & \\
\hline ListSelect & $\nabla$ & \\
\hline TextBox & $\nabla$ & $\nabla$ \\
\hline Title & $\nabla$ & $\nabla$ \\
\hline Relative3DStream & $\nabla$ & $\nabla$ \\
\hline Relative2DStream & $\nabla$ & \\
\hline
\end{tabular}




\begin{tabular}{r|c|c|}
\hline Direction4 & $\nabla$ & \\
\hline Chars & $\square$ & \\
\hline Image2DView & $\square$ & $\square$ \\
\hline
\end{tabular}

An image of the .NET windows forms implementation, connected to a PowerPoint controller service, is provided in Figure 14: Windows Forms Client, below.

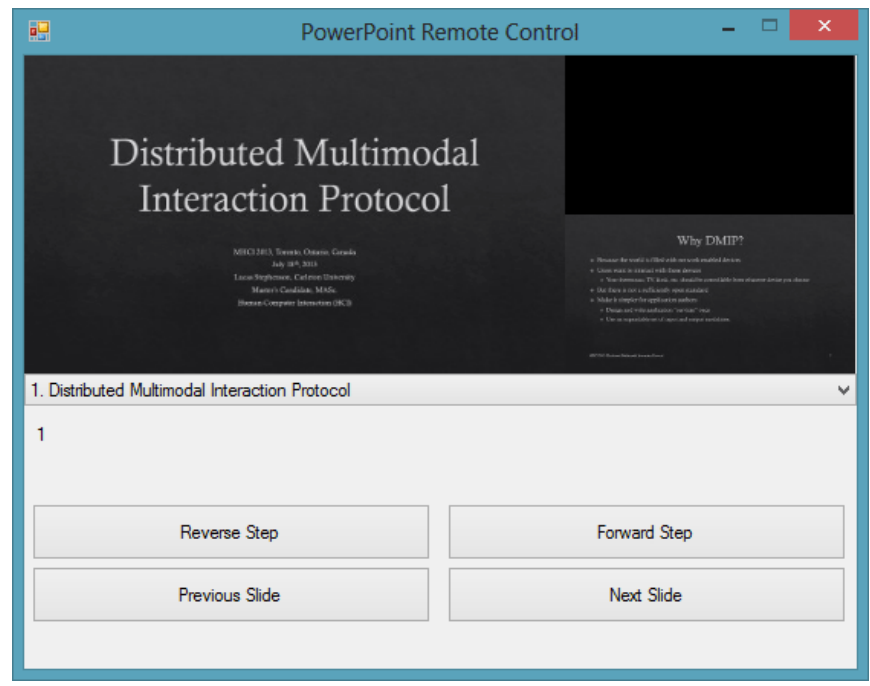

Figure 14: Windows Forms Client

The implementation of the client layer provided a validation of the DMIP protocol, and programming reference for projects that intend to use DMIP.

\subsubsection{Implemented Services}

A .NET API supporting the authoring of services was created, this API provides common functionality for services and is divided across 2 packages; Service and Library.

The Service package provides functionality for basic service implementations; it provides session handling, basic layout definition from XML-format (7.2.6.1 XML Layout Language) files, basic channel type compatibility checking.

The Library package provides additional augmentation/utility methods for services to ease repetition of some common service coding tasks and make service development simpler, 
including transport of client compatible layouts, clearing/switching of layouts and methods to broadcast DMIP payloads to all connected clients.

A number of simple sample service applications were created to help develop the protocol

itself, test a variety of scenarios and help the formulation of Channel Types. These sample services are available as a reference, and are included in the .NET DMIP implementation source, available: http://iv.csit.carleton.ca/ dmip/. The sample services created are described and discussed in the proceeding sections; Thermostat (section 7.2.5.3), PowerPointController (section 7.2.5.4), NumberGuess (section 7.2.5.5), and WindowsMouseControl (section 7.2.5.6).

\subsubsection{Thermostat Service}

The Thermostat Service was created as an initial exploratory prototype upon which to explore and test DMIP. It allows a user to see a current temperature (simulated) and adjust the target temperature. The service simulates automatic cooling/heating and keeps the connected client(s) updated.

The thermostat service defines a layout fully from an XML-layout file (7.2.6.1 XML Layout Language). It uses the channel types Label2D and SingleState2DButton to allow basic interaction with the service. Optionally, a Title channel is used to control the application's title. Figure 15: Thermostat Service shows the windows form client connected to a thermostat service. 


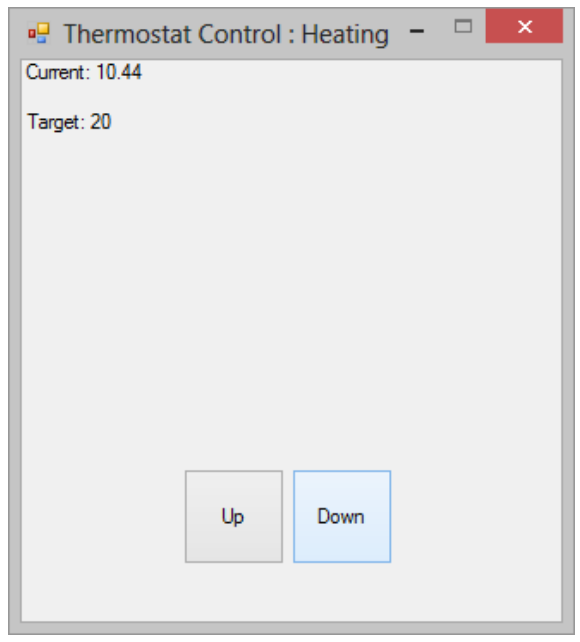

Figure 15: Thermostat Service

Development of the Thermostat service aided the evolution of DMIP and provided initial validation that the Channel Types scheme was valid. It also drove the development of an XML format layout language (7.2.6.1 XML Layout Language). It now provides a basic example which can be used by others to learn how to use the protocol and provided APIs.

\subsubsection{PowerPoint Controller Service}

The purpose of the development of PowerPoint Controller Service application was twofold; create a directly useable application that could be used by the researcher, and peers, and to verify that DMIP could be used to create applications similar to Eric Torunski's [20] PowerPoint controller application. The application allows the control of a PowerPoint slide show.

As part of the development of this service additional Channel Types were created and of metachannels (section 5.5.5) were introduced to reduce Channel definition duplication and to provide flexibility for channels.

The PowerPoint Controller Service application was created as a .NET PowerPoint Add-In, and makes use of Visual Studio Tools for Office ${ }^{2}$. An XML file is used to define a standard layout for all clients. The XML file provides positioning, interaction and text label information for relevant

\footnotetext{
${ }^{2}$ http://msdn.microsoft.com/en-us/library/d2tx7z6d(v=vs.120).aspx
} 
channel instances, as well as providing what channel types are required or optionally used by the connecting clients. A view of the service from the windows forms client is seen in Figure 14: Windows Forms Client, and from the android client in Figure 16: PowerPoint Controller from Android.

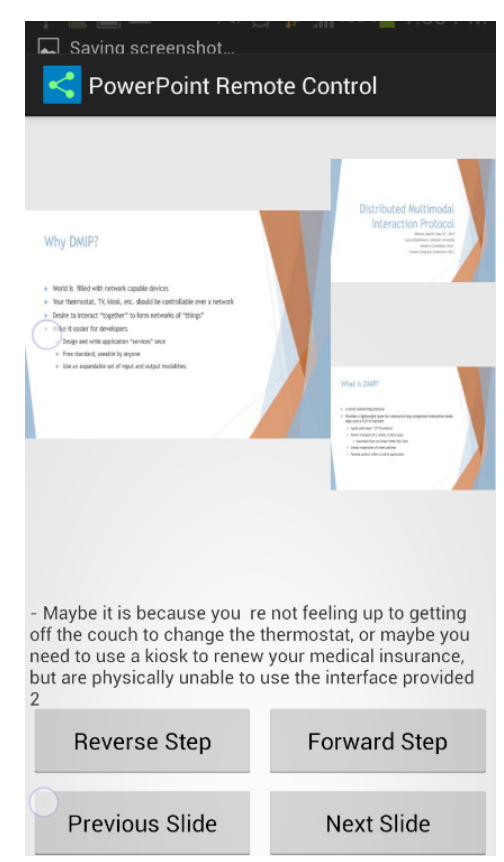

Figure 16: PowerPoint Controller from Android

The channels Label2D and SingleState2DButton are required and enable the client to display slide navigation buttons, and allow viewing the slide notes. Optionally the service supports Direction4 to enable arrow key navigation, Title to provide a title caption for the client application, Image2DView that enables a view of the current, previous and next slides in the PowerPoint slide deck and ListSelect which gives a selectable list of slides that can be navigated to. When a compatible client connects, it is provided with an interface generated by the service. The network sequence diagram below in figure 4, shows the network messages used for the "Previous" button, within the application. 


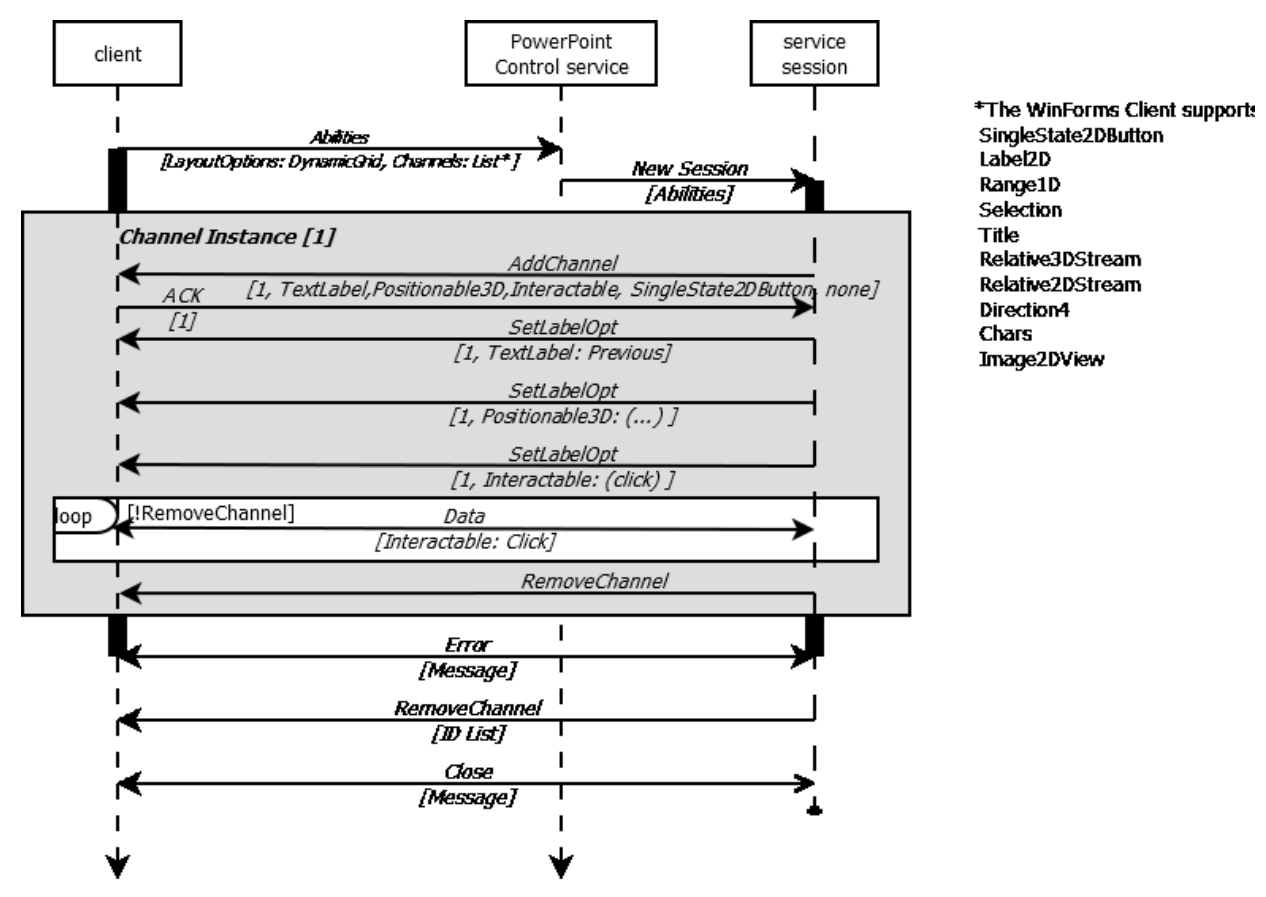

Figure 17: Network Sequence for Previous Slide

\subsubsection{Number Guess Service}

The Number Guess Service was created to test multi-user scenarios. The service allows users to join the game, each round the service randomly selects and integer and allows users to guess the number. The specific user, by way of the client is informed if their guess is too high or too low (or correct). Only one client is allowed to guess at a time, and the clients guess in sequence until the number is guessed.

The Number Guess service was the first service created that required text/number input and thus the TextBox channel type was developed, and the TextLabel meta-channel was introduced to centralize Channel Type text data. The Enabled meta-channel was also developed to provide a channel for visually enabling/disabling channels on clients.

Figure 18: Number Guess Service Progression shows the basic progression of a "game" in the number guess service. Label 1 shows the initial client layout that allows users to input a name. Label 2 shows the second layout that asks the user to enter a guess, but the "Guess" button is disabled (via the Enabled meta-channel) because a round is not started (or it is not their turn). 
Label 3 shows the service application, allows for a round to be started. Label 4 shows the "Guess" button enabled, and Labels 5 and 6 show the win condition state on the client and service respectively.

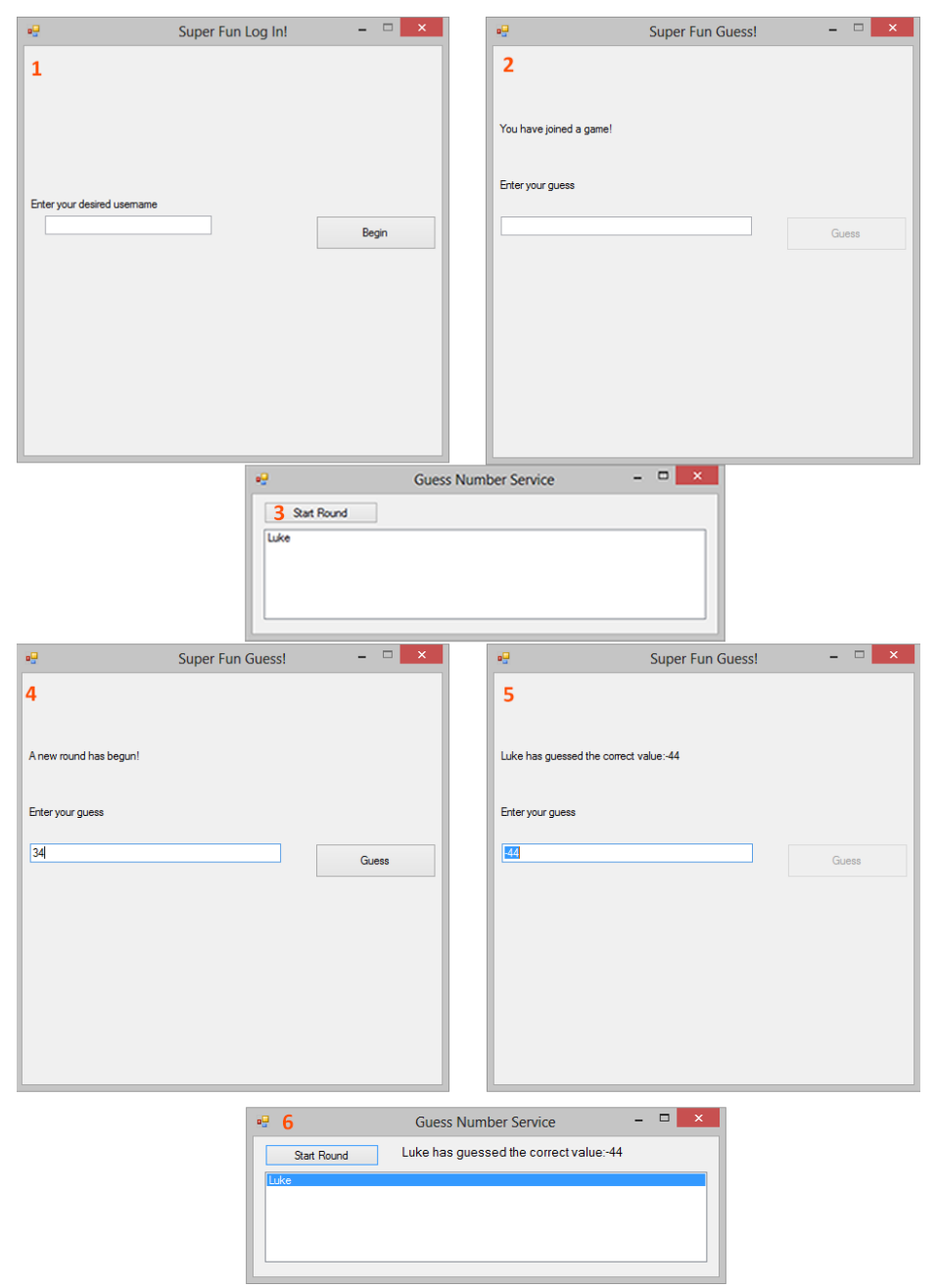

Figure 18: Number Guess Service Progression

To support multi-user scenarios, functionality was added to the service API to allow messages to be broadcast to service connected clients. The service provides two layouts (login and play) and thus switching of client layouts was developed for the service library package. The development of this service provides validation of the possibility of handling multi-user scenarios and a useable example thereof. 


\subsubsection{Windows Mouse Control Service}

The Windows Mouse Control Service was created to test UDP channel data. The service allows users to control the mouse on the service computer. Buttons are provided to emulate the buttons on a mouse, and mouse position is controlled through either a Relative3DStream or Relative2DStream channel. For timeliness UDP transport is used if the connection supports it.

The Android client implements Relative3DStream by way of the accelerometer, the service merely takes data and moves the mouse on the service computer's screen based on the data provided by the Relative3DStream Data payloads.

This implementation is not useable with windows forms client, as the client buttons are not selectable without moving the mouse, the default Data source for Relative2DStream. Alternatively, the client would capture the local machine's mouse movements and instead of mouse clicks, use key presses, using the Chars channel.

A view of the Android client, connected to a Windows Mouse Control service is provided below, in Figure 19: Windows Mouse Control Service, Android Client. 


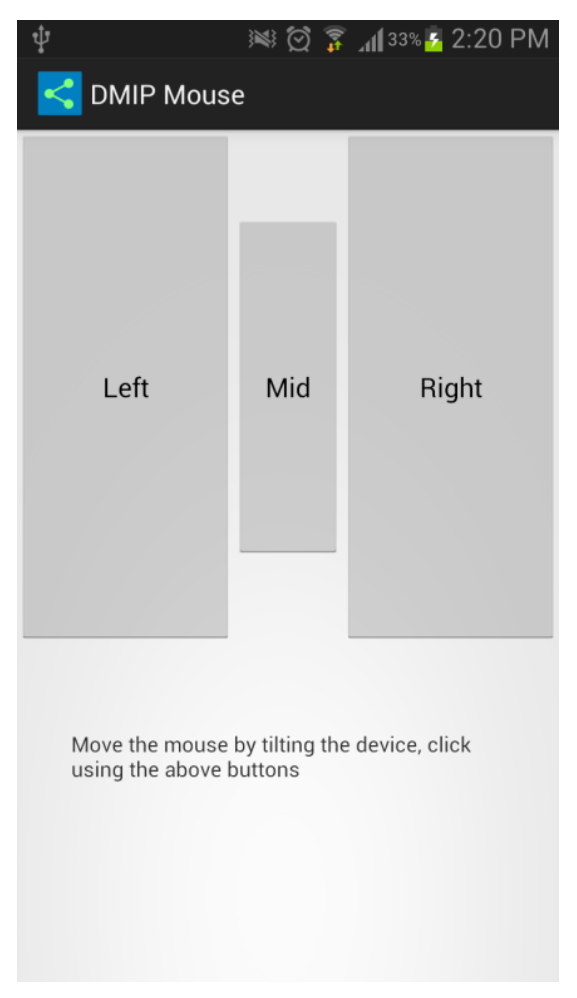

Figure 19: Windows Mouse Control Service, Android Client

\subsubsection{Protocol Support and Augmentation}

The implementation sample services application developed shared some common functionality. In order to minimize the effort required to author DMIP services tools and methods were made generally accessible, so as to be part of the implementation's API. This functionality is not part of DMIP itself but can aid in the development of future services it is discussed in 7.2.5.2, Implemented Services. Design of client interfaces within code can be overbearing and tedious, an XML layout engine was therefore developed, it is described in section 7.2.6.1, below.

\subsubsection{XML Layout Language}

Creating graphical layouts from code is tedious for the developer. While the creation of a graphical tool would be ideal, it was beyond the scope of this thesis. Progression towards a graphical tool for interface designers, and centralizing layout design was made by defining of an 
XML file format for services. This file format is simpler to maintain than code sections. A sample layout XML file (layout file) is available in Appendix B: PowerPoint Controller Show XML.

The requirements section of the layout files specify what Channel Types are required by the client. Additionally, optional Channel Types are also specified. The requirements section allows the service API layer to automatically evaluate compatibility with incoming client Abilities (section 5.6.2.5) payloads. In layout file fragment below, it is specified that Label2D and SingleState2DButton channels are required by the client to interact with the service. Additionally 3 optional ChannelTypes are specified (Title, Relative2DStream and Relative3DStream), this allows the API layer to notify implementing service applications what relevant Channel Types are available.

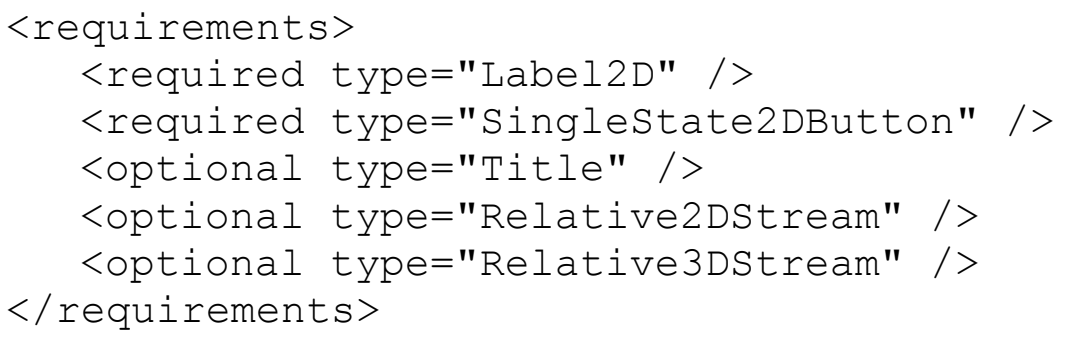

Channel sections represent instances of Channel Types that are to be added to the client by the service. Enclosed within the channel sections are optional metachannel and options sections, these contain the information that is to be sent by the service as part of initial SetChannelOpt and AddChannel messages. The following file fragment specifies that a SingleState2DButton be should be placed on the client's display. The button is centered using the Positionable3D Meta Channel Type, 20\% from the vertical edges, and 30\% from the horizontal. It has a label, of "Hello" specified by the TextLabel Meta Channel Type. It also must send messages according to the Interactable Meta Channel Type when the button is pushed, or released. 


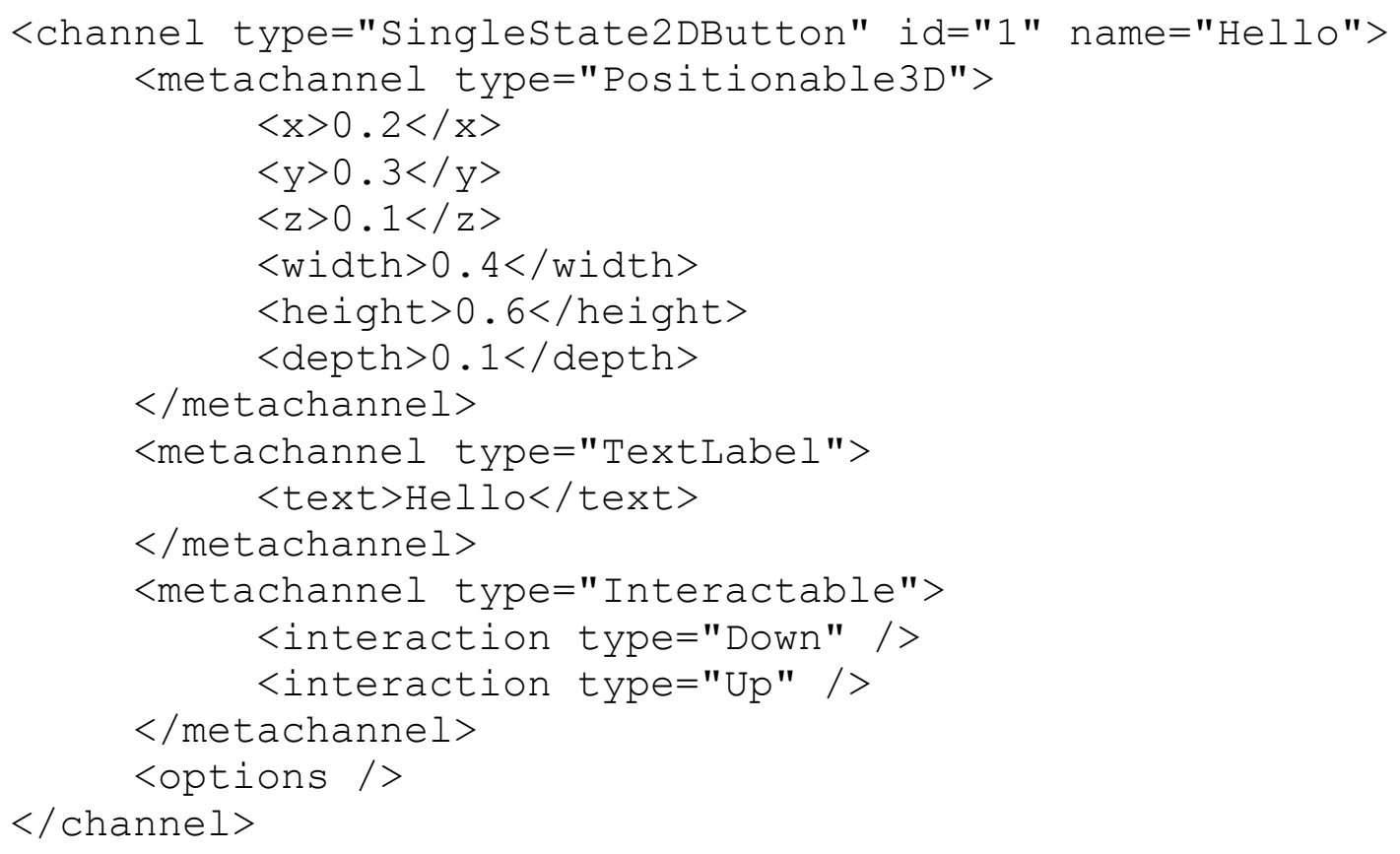

The resultant channel is shown in the Windows Forms client implementation, in Figure 20:

Sample XML Layout File Result.

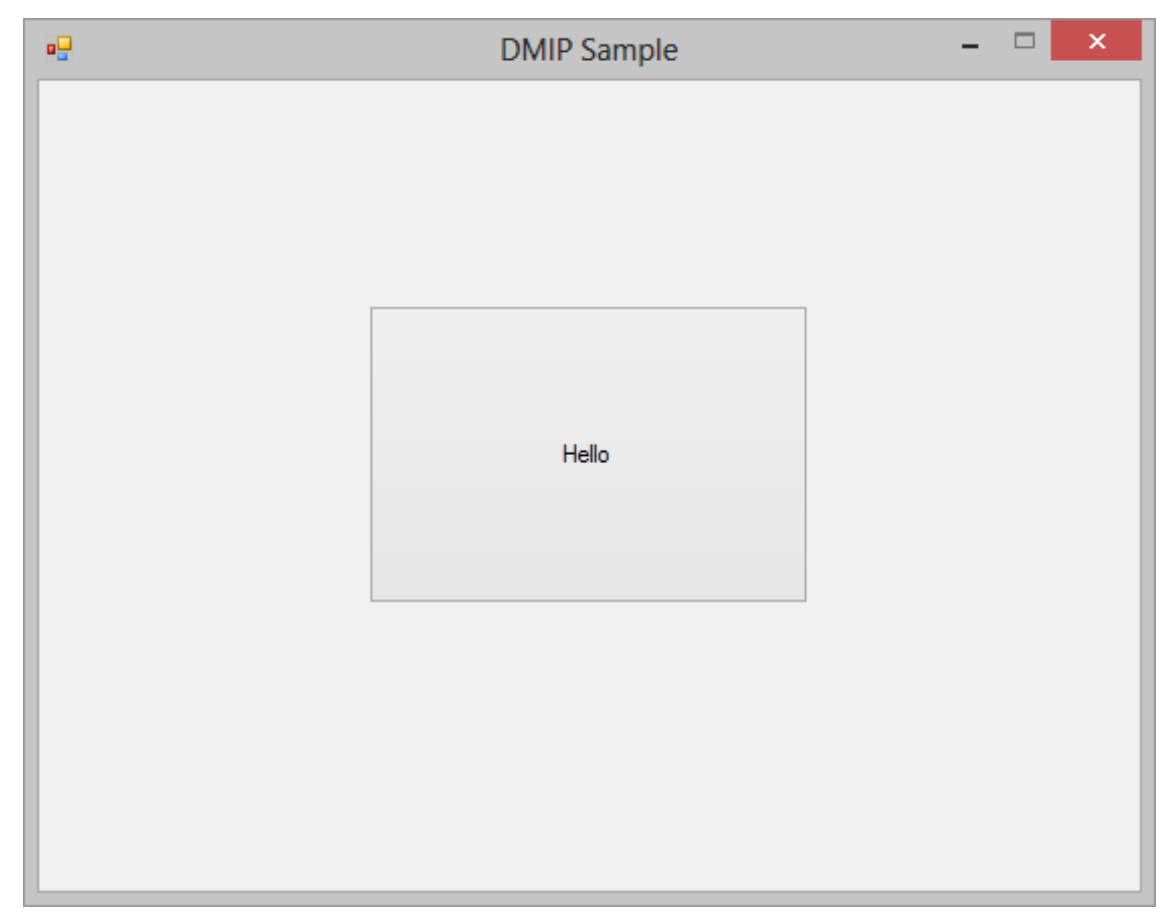

Figure 20: Sample XML Layout File Result 


\section{Discussion}

DMIP currently provides a simplified development path to enable networked devices to utilize network based services. Every effort has been made to validate DMIP, it has been validated for several basic scenarios (section 7.2) however as a new technology there exists the possibility that there are limitations. Section 8.2, discusses some potential limitations of DMIP. In order to support and strengthen DMIP solutions further, related work is discussed and proposed in section 8.3, Future Work.

\subsection{Value and Testing}

DMIP is a tool to aid authors of applications by providing them with a platform that reduces concerns over networking and disparate device user-interaction capabilities. This, in turn can provide greater access to the technology to the current and potential users of their applications by making them immediately available to a greater number of client devices and providing a flexible, yet consistent interface across devices. Testing usability of applications developed with the tool is beyond the scope of this work, application design, while facilitated with DMIP, remains part of the application development process. DMIP provides an application layout and data-flow design platform. By targeting one effective platform, application authors can design, deploy, evaluate and maintain a single application providing a higher return on investment and additional resources for additional development. While effort has been made to communicate with industry and interest has been shown, time limitations have reduced the ability to evaluate the platform directly with developers.

\subsection{Limitations}

Despite extendibility, standardization of additional channel types can enable application developers to more simply make use of more interaction modalities in their applications potentially making the resultant applications more appealing to end users. Extension channel types allow " $3^{\text {rd }}$-party" channel types to be created, however it is unlikely that they will become widespread without standardization centralizing the definition of novel channels. 
Another potential improvement is security; while the underlying transport layer could provide connection encryption [30] using a transport layer security scheme, it might be valuable to encrypt specific channel instances and have protocol handling of security measures so that users can view and manipulate the security information and/or to optimize transport of such information.

DMIP relies on a TCP/IP based network to operate, as such, it is not directly compatible with other remote technologies that do not include the TCP/IP stack. Lower level transport mechanisms, such as Bluetooth, near field communication (NFC) and others could be used to transport interaction data, but DMIP does not directly support these technologies.

It is possible that DMIP clients can support some channel types in different (multiple input mechanisms) ways, or the same input could implement multiple channel types. For example the Windows Mouse Control service (section 7.2.5.6), could potentially be provided the target mouse co-ordinates in multiple ways, remote mouse co-ordinates, gaze tracking, accelerometer, or any other input that could be provided in at least 2 dimensions. Currently, the service application chooses between multiple channel types, whereas in the situation that multiple client inputs could implement a channel type, it is left to the client developer to choose, or provide configuration options so that the user can decide.

\subsection{Future Work}

To build upon and improve the DMIP specification, and supporting technologies developed for this thesis, additional future work is proposed. The proceeding sections describe proposed work. Section 8.3.1, Multi-User Scenarios discusses the use of DMIP to support multiple simultaneous users. Section 8.3.2, Additional Implementation Features, describes additions that could augment the API developed as part of the evaluation implementation (Section 7.2). Section 8.3.4, Integration with Other Technologies, discusses integration with other modality aggregation techniques as introduced in section 2.4. Section 8.3.5 Standardization details 
rationale and proposal for a means of standardizing DMIP and the supported Channel Types. Service location, allowing automatic detection of DMIP services by clients is discussed in section 8.3.6. Service Endpoint Location and integration with popular web technologies, is discussed in section 8.3.7 Transport Over HTTP and Websockets. And section 8.3.8, User Friendly Clients discusses the opportunity for a variety of client DMIP implementing applications.

\subsubsection{Multi-User Scenarios}

It is simple to imagine DMIP working in multi-user scenarios, in fact perhaps most, if not all scenarios can make use of multiple simultaneous client connections. While the DMIP protocol focuses on a single Client-Service session, services are expected to be designed to support multiple sessions. The number guessing game implementation (section 7.2.5.5) was created to test the feasibility of this type of scenario. The other evaluation implementations created and discussed in section 7.2.5 all work with multiple connected clients. However the number guessing game manages a shared state for all connected clients, this functionality rests within the service implementation's code and the .NET service API, briefly discussed in section 7.2.6. While ability for DMIP to support and enable multi-user scenarios is verified, it is suggested that further examples and testing be completed, so that best practices can be compiled and the service implementation API can be further developed.

\subsubsection{Additional Implementation Features}

The service implementation API described in section 7.2.5.2 provides developers with common service functionality to make development of services implementing DMIP simpler and faster. Implementing additional services would provide discovery of common features that could be implemented in a re-useable API. One example feature that could be implemented would be notification of incoming SplitPayload messages, so that partial data can be processed and progress messages could be provided to end users on DMIP endpoints. 


\subsubsection{Multi-User Scenarios}

It is simple to imagine DMIP working in multi-user scenarios, in fact perhaps most, if not all scenarios can make use of multiple simultaneous client connections. While the DMIP protocol focuses on a single Client-Service session, services are expected to be designed to support multiple sessions. The number guessing game implementation (section 7.2.5.5) was created to test the feasibility of this type of scenario. The other evaluation implementations created and discussed in section 7.2.5 all work with multiple connected clients. However the number guessing game manages a shared state for all connected clients, this functionality rests within the service implementation's code and the .NET service API, briefly discussed in section 7.2.6. While ability for DMIP to support and enable multi-user scenarios is verified, it is suggested that further examples and testing be completed, so that best practices can be compiled and the service implementation API can be further developed.

\subsubsection{Integration with Other Technologies}

The related technologies section (2.4) discusses several related technologies that provide methods of codifying, aggregating and consuming multimodal user data. DMIP Channel Types could be created to support each these technologies by allowing transport of encapsulated classified data.

\subsubsection{Standardization}

The authors of this work will be evaluating options for the standardization of DMIP. The goal of standardization is to facilitate greater adoption of the technology and to aid in providing a consistent target for application authors. However, standardization can take a long time, it is therefore planned that Channel Types be removed from the formal standard and instead be standardized and through less time-intensive process to allow faster reactions to changes in the available interaction modalities. To enable the use of modalities not currently supported by the existing Channel Types there exists the need to define community accepted means of representing various modes. A standardization procedure needs to be created to support the introduction of new transportable DMIP Channel Types. 


\subsubsection{Service Endpoint Location}

To make the location and consumption of DMIP services simpler for end users, integration with service location protocol (SLP) is proposed. SLP allows the location of service endpoints on local networks [24]. A preliminary study was conducted to explore the feasibility of using SLP to locate network endpoints for novel services, and it was successful ${ }^{3}$. A DMIP client able to manage connect-able services and provide service endpoint location using SLP is therefore proposed, in order to improve user experience by increasing accessibility of DMIP services.

\subsubsection{Transport Over HTTP and Websockets}

Higher level TCP/IP stack protocols, such as the Hypertext Transfer Protocol (HTTP) combined with Websockets (WS) could be used to transport DMIP-like data, and could provide opportunities for creating clients for web browsers if a DMIP-to HTTP/WS layer were created. It is imagined that such clients would be able to provide flexible interaction services over the internet and could be used to offer alternative/augmented ways of navigating the World Wide Web.

\subsubsection{User Friendly Clients}

Despite considerations for things like SLP, there is further need for client implementations to have their user experience evaluated and designed. Additional helpful features can be imagined, configuration options and common endpoints are immediate candidates for requirements lists. DMIP offers a method of negotiating, providing and consuming multimodal data, however the design client applications is not defined. Future work should include a focus on creating guidelines for DMIP client authors.

\footnotetext{
${ }^{3}$ http://iv.csit.carleton.ca/ dmip/files/SLP.pptx Implementing Service Location Protocol
} 


\section{Conclusion}

There is user desire to use mobile devices as "universal remote" controls, as it enables them to access technology in new ways. Users that could not previously use a technology based service, by not having access to specific equipment or because of a physical disability can use a broader range of devices and human input and output devices if the technologies involved support DMIP. Giving more ubiquitous access to consume and control technology is the purpose of DMIP. For DMIP to become pervasive so that users can use the technology around them it needs to have/support:

- An expanding list of input/output modalities

- Support for different configurations/combinations of these modalities

- Be an accessible and open (free) standard

- Have tools and sample code accessible to developers

- Have tools for designers so that they can create useable interface designs

- Be useable and simple to use for end users.

By providing the extensible modality system, DMIP can potentially support any humancomputer interaction modality (Section 7.1). DMIP also provides basic negotiation of these modalities so that a dynamic set of remote client modalities can be used to interact with a service application (section 5.5). DMIP is released to be freely and openly used by anyone, and is provided with a permissive MIT license. Developing software that makes use of distributed clients is much simplified by reducing concern about the client's operating system, hardware abilities and simplified network programming, additionally with the sample implementations developers have tools, including sample code that can be used as a starting point so that developers can more easily create DMIP capable services. An XML based file format has been defined so that interfaces can be laid out external to service code (section 7.2.6.1). User interface designer tools for DMIP are proposed, and could be developed by the public, given the open-standard nature of the protocol. Beyond the core goal of DMIP, to provide a useable 
interface for technology, the end user's needs are not directly addressed (section 8.3.8) but there exists opportunity for clients to be developed for many different devices and platforms.

The question posed in 2.1 Research Question:

There is an ever-increasing ability for the user to generate data on personal devices, can this data be simply consumed by remote computer devices to provide an interactive user based experience for potentially any capable remote technology?

Is answered and proven positive with the designed and evaluated protocol, DMIP. DMIP can enable ubiquitous access to technology by providing an open standard means of negotiating and transporting multi-modal interaction data. DMIP is a tool to help enable new scenarios, and to increase access to existing ones. Although DMIP provides many features, it is at most a single piece of any human-computer interaction. The implementations provided herein were created primarily to aid in evaluating the protocol and to provide samples to interested parties. DMIP is an enabling technology, or tool, and can be used in a potentially limitless number of scenarios. DMIP aims to help application authors create new experiences for users. DMIP Implementation and source code examples are available: http://iv.csit.carleton.ca/ dmip/. 


\section{References}

[1] L. Nigay and J. Coutaz, "A design space for multimodal systems: concurrent processing and data fusion," in Human factors in computing systems , New York, 1993.

[2] W. R. Stevens, TCP/IP Illustrated: the protocols, Boston, Massachsetts: Addison-Wesley Professional, 1994.

[3] Rogers Communications, "Rogers Nextbox 2.0," [Online]. Available: http://www.rogers.com/web/content/Rogers-Nextbox2. [Accessed 15 July 2013].

[4] J. Rempel, "Control Your Thermostat from Almost Anywhere? There's an App for That: An Evaluation of the Nest Thermostat," AFB AccessWorld ${ }^{\circledR}$ Magazine, vol. 13, no. 12, December 2012.

[5] J. Nielson and R. Molich, "Heuristic evaluation of user interfaces," Seattle, WA, 1990.

[6] Quinstreet Enterprise, "What is network? - A Definition From the Webopedia Computer Dictionary," [Online]. Available: http://www.webopedia.com/TERM/N/network.html. [Accessed 20 June 2013].

[7] T. Markku, H. Soronen, S. Pakarinen, J. Hella, T. Laivo, J. Hakulinen, A. Melto, J.-P. Rajaniemi, T. Miettinen, E. Mäkinen, T. Heimonen, J. Rantala, P. Valkama and R. Raisamo, "Accessible Multimodal Media Center Application for Blind and Partially Sighted People," Computers in Entertainment (CIE) - Theoretical and Practical Computer Applications in Entertainment, vol. 8, no. 3, p. Article 16, December 2010.

[8] P. Cohen and S. Oviatt, "Perceptual user interfaces: multimodal interfaces that process what comes naturally," Communications of the ACM, vol. 43, no. 3, pp. 45-53, 2000.

[9] A. Taivalsaari and T. Mikkonen, "The Web as an Application Platform: The Saga Continues," in Software Engineering and Advanced Applications (SEAA), Oulu, Finland, 2011.

[10] S. K. Card, J. D. Mackinlay and G. G. Robertson, "The design space of input devices," in CHI '90 Proceedings of the SIGCHI Conference on Human Factors in Computing, Seattle, USA, 1990.

[11] M. Kaltenbrunner, "reacTIVision and TUIO: A Tangible Tabletop Toolkit," in Interactive Tabletops and Surfaces, Banff, Canada, 2009. 
[12] A. Wu, J. Jog, S. Mendenhall and A. Mazalek, "A Framework Interweaving Tangible Objects, Surfaces and Spaces," in HCI International, Orlando Florida, USA, 2011.

[13] J. Shen, S. Wenzhe and M. Pantic, "HCl 2 Workbench A development tool for multimodal human-computer interaction systems," in Automatic Face \& Gesture Recognition and Workshops (FG 2011), Santa Barbara, CA, 2011.

[14] M. Johnston, "Building multimodal applications with EMMA," in 2009 international conference on Multimodal interfaces, Beijing, China, 2009.

[15] OpenNI, "OpenNI: Introduction," OpenNI, 27 November 2011. [Online]. Available: http://openni.org/Documentation/Reference/introduction.html. [Accessed 14 September 2012].

[16] K. Bonhyun, A. Taewon, I. JungSik, P. Youngsuk and S. Taesgik, "R-URC: RF4CE-Based Universal Remote Control Framework Using Smartphone," in Computational Science and Its Applications (ICCSA), Fukuoka, Japan, 2010.

[17] M. Kaltenbrunner, T. Bovermann, R. Bencina and E. Costanza, "TUIO - A Protocol for Table-Top Tangible User Interfaces," in 6th International Workshop on Gesture in HumanComputer Interaction and Simulation, Vannes, France, 2005.

[18] H. Ishii and B. Ullmer, "Tangible bits: towards seamless interfaces between people, bits and atoms," in SIGCHI conference on Human factors in computing systems (CHI '97), Atlanta, Georgia, USA, 1997.

[19] J. Shen and M. Pantic, "A software framework for multimodal humancomputer interaction systems," in IEEE International Conference on Systems, Man, and Cybernetics, San Antonio, TX, USA, 2009.

[20] E. Torunski, A. El Saddik and E. Petriu, "Gesture recognition on a mobile device for remote event generation," in Multimedia and Expo (ICME), Barcelona, 2011.

[21] J. Nielson, "Iterative user-interface design," Computer, vol. 26, no. 11, pp. 32-41, 1993.

[22] N. Johansson, M. Kihl and U. Körner, "TCP/IP over the Bluetooth Wireless Ad-hoc Network," Department of Communication Systems, Lund University, Lund, Sweden, 2003.

[23] L. M. de Sales, H. O. Almeida and A. Perkusich, "On the performance of TCP, UDP and DCCP over 802.11 g networks," in ACM Symposium on Applied Computing, Ceará, Brazil, 2008.

[24] E. Guttman, C. Perkins, J. Veizades and M. Day, "Service Location Protocol, Version 2," June 1999. [Online]. Available: http://tools.ietf.org/html/rfc2608. 
[25] E. Guttman, C. Perkins and J. Kempf, "Service Templates and Service: Schemes," June 1999. [Online]. Available: http://tools.ietf.org/html/rfc2609.

[26] R. Braden, "Requirements for Internet Hosts -- Communication Layers," October 1989. [Online]. Available: http://tools.ietf.org/html/rfc1122.

[27] S. Bradner, "Key words for use in RFCs to Indicate Requirement Levels," March 1997. [Online]. Available: http://tools.ietf.org/html/rfc2119. [Accessed 10 July 2013].

[28] J. Nielson, "10 Usability Heuristics for User Interface Design," 1 January 1995. [Online]. Available: http://www.nngroup.com/articles/ten-usability-heuristics/. [Accessed 9 March 2013].

[29] N. O. Bernsen, "Modality theory in support of multimodal interface design," in ERCIM Workshop on Multimodal Human-Computer Interaction, Roskilde, Denmark, 1994.

[30] T. Dierks and E. Rescorla, "The Transport Layer Security (TLS) Protocol, Version 1.2," August 2008. [Online]. Available: https://tools.ietf.org/html/rfc5246. [Accessed 2 February 2013].

[31] D. Mills, U. Delaware, J. Martin, J. Burbank and W. Kasch, "Network Time Protocol Version 4: Protocol and Algorithms Specification," June 2010. [Online]. Available: http://tools.ietf.org/html/rfc5905. [Accessed 3 September 2012].

[32] W3C, "The WebSocket API," 29 September 2011. [Online]. Available: http://www.w3.org/TR/websockets/. [Accessed 21 November 2011]. 
Appendix A: Interaction Ledger

Survey

Available: http://lucasstephenson.net/hcin5909/

Results

\begin{tabular}{|c|c|c|c|c|c|}
\hline $\begin{array}{r}\text { Interaction } \\
\text { Object }\end{array}$ & Purpose & Actions Performed & Best Simulated & Motion & Selection \\
\hline car & opening, closing locking etc & $\begin{array}{l}\text { I use a smart key which } \\
\text { means that my door can be } \\
\text { locked and unlocked as long } \\
\text { as I carry the key in my purse } \\
\text { or pocket - }\end{array}$ & $\begin{array}{l}\text { ? I am not sure } \\
\text { what to put here }\end{array}$ & $\begin{array}{c}\checkmark \\
\text { User does a } \\
\text { wrist motion }\end{array}$ & $\begin{array}{c}\checkmark \\
\text { User selects key and } \\
\text { points to lock }\end{array}$ \\
\hline $\begin{array}{c}\text { computer } \\
\text { mouse. }\end{array}$ & $\begin{array}{l}\text { interacting with the object to } \\
\text { perform desktop tasks. } \\
\text { accomplishing: getting } \\
\text { around the desktop and } \\
\text { selecting pages I wish to see, } \\
\text { i.e.: i would like to open a } \\
\text { file so double click to open; } \\
\text { or I would like to see below, } \\
\text { so I scroll. }\end{array}$ & $\begin{array}{l}\text { Actions: } 1 \text { - move, } 2 \text { - click, } \\
\text { and } 3 \text { - scroll } \\
\text { anticipated results: } 1 \text { - as i } \\
\text { move the mouse, the cursor } \\
\text { also moves on the computer } \\
\text { screen, } 2 \text { and } 3 \text {-- as i click } \\
\text { and scroll i expect a change } \\
\text { within the interface. }\end{array}$ & & $\begin{array}{c}\checkmark \\
\text { Move mouse } \\
\text { cursor }\end{array}$ & $\begin{array}{c}\checkmark \\
\text { Select items by } \\
\text { clicking mouse }\end{array}$ \\
\hline $\begin{array}{r}\text { Keyboard } \\
\text { attached to } \\
\text { my laptop }\end{array}$ & $\begin{array}{l}\text { filling in this form. lol. } \\
\text { However, entering text in } \\
\text { any way necessary (word } \\
\text { documents, emails, } \\
\text { facebook, etc.) }\end{array}$ & $\begin{array}{l}\text { What I did to the object was } \\
\text { depress keys. The anticipated } \\
\text { result was that the key I } \\
\text { depressed would result in the } \\
\text { corresponding character or } \\
\text { symbol being displayed on }\end{array}$ & $\begin{array}{l}\text { touch-screen key } \\
\text { pad on screen. } \\
\text { That\'s all I've } \\
\text { really got for this } \\
\text { one. }\end{array}$ & & $\begin{array}{c}\checkmark \\
\text { Select items on } \\
\text { screen, select keys }\end{array}$ \\
\hline
\end{tabular}




\begin{tabular}{|c|c|c|c|c|c|}
\hline & & $\begin{array}{l}\text { the screen in the order in } \\
\text { which I pressed the keys. }\end{array}$ & & & \\
\hline Spoon & $\begin{array}{l}\text { using it to eat my breakfast } \\
\text { (oatmeal) }\end{array}$ & $\begin{array}{l}\text { I picked up the spoon out of } \\
\text { the cutlery drawer, and } \\
\text { placed it into my bowl of } \\
\text { oatmeal. I then used the } \\
\text { spoon to scoop the oatmeal } \\
\text { out of the bowl and into my } \\
\text { mouth. The anticipated result } \\
\text { is that when I scoop the food, } \\
\text { it stays in the spoon and } \\
\text { doesn 't fall off, until I move } \\
\text { the spoon up to my mouth to } \\
\text { eat. }\end{array}$ & $\begin{array}{l}\text { Good question. } \\
\text { Currently, holding } \\
\text { down the ctrl } \\
\text { button while } \\
\text { selecting objects } \\
\text { with a mouse } \\
\text { offers the } \\
\text { individual the } \\
\text { ability to \"scoop\" } \\
\text { several objects at } \\
\text { once. However, } \\
\text { this may not be the } \\
\text { best metaphor. } \\
\text { Maybe just } \\
\text { dragging a mouse } \\
\text { over several } \\
\text { objects and they } \\
\text { are automatically } \\
\text { selected (similar to } \\
\text { how the swype } \\
\text { keyboard input } \\
\text { works on Samsung } \\
\text { phones) }\end{array}$ & $\begin{array}{c}\checkmark \\
\text { Scoop }\end{array}$ & $\begin{array}{c}\checkmark \\
\text { Select multiple } \\
\text { objects by } \\
\text { grasping/pointing }\end{array}$ \\
\hline $\begin{array}{l}\text { Playstation } \\
\text { controller }\end{array}$ & $\begin{array}{l}\text { I am using this object to } \\
\text { control what is going on with } \\
\text { the playstation e.g. selecting } \\
\text { a video to watch, selecting a } \\
\text { game to play, making my } \\
\text { character on screen do }\end{array}$ & $\begin{array}{l}\text { I picked up the controller, } \\
\text { and started pressing buttons } \\
\text { which corresponded with } \\
\text { what I wanted to happen on } \\
\text { screen (I wanted to scroll to } \\
\text { the right to select video }\end{array}$ & $\begin{array}{l}\text { The most similar to } \\
\text { this would be } \\
\text { scrolling with the } \\
\text { mouse. }\end{array}$ & & $\begin{array}{c}\checkmark \\
\text { User pressed buttons }\end{array}$ \\
\hline
\end{tabular}




\begin{tabular}{|c|c|c|c|c|c|}
\hline & $\begin{array}{l}\text { various tasks within a video } \\
\text { game, etc. }\end{array}$ & $\begin{array}{l}\text { games, so I pressed the pad } \\
\text { right button several times). } \\
\text { The anticipated result was } \\
\text { that the focus on the screen } \\
\text { would move in the direction I } \\
\text { pressed on the pad. }\end{array}$ & & & \\
\hline Can opener & Opening a can & $\begin{array}{l}\text { cranked the know? it went } \\
\text { around in a circle and opened } \\
\text { the can }\end{array}$ & & $\begin{array}{c}\checkmark \\
\text { Rotation }\end{array}$ & \\
\hline boots & To put on my boots & $\begin{array}{l}\text { 1. untied my laces } \\
\text { 2. put my foot in the boot } \\
\text { 3. pulled the boot up onto } \\
\text { my foot }\end{array}$ & & $\begin{array}{c}\checkmark \\
\text { Complex } \\
\text { gestures }\end{array}$ & \\
\hline stove/burner & heating water on the stove & $\begin{array}{l}\text { turned the knob on the stove } \\
\text { to reach the right } \\
\text { temperature }\end{array}$ & & & $\begin{array}{c}\checkmark \\
\text { Select temperature }\end{array}$ \\
\hline litter box & clean it & $\begin{array}{l}\text { scoop the poop with a } \\
\text { scooper }\end{array}$ & & $\begin{array}{c}\checkmark \\
\text { Scoop }\end{array}$ & \\
\hline phone & calling my parents & pushing buttons & $\begin{array}{l}\text { talking to the } \\
\text { phone telling it } \\
\text { who I want to call } \\
\text { without picking up } \\
\text { the phone or } \\
\text { dialing. }\end{array}$ & & $\begin{array}{c}\checkmark \\
\text { Select numbers on } \\
\text { device }\end{array}$ \\
\hline light switch & $\begin{array}{l}\text { turning on the lights to turn } \\
\text { on the christmas lights }\end{array}$ & flipped the switch on the wall & $\begin{array}{l}\text { talk to the tree or } \\
\text { press a button on } \\
\text { my phone }\end{array}$ & & $\begin{array}{c}\checkmark \\
\text { Press } \\
\text { switch/buttons/voice } \\
\text { selection }\end{array}$ \\
\hline ignition & turning on my car & $\begin{array}{l}\text { put the key in the ignition } \\
\text { and turning it }\end{array}$ & & $\begin{array}{c}\checkmark \\
\text { User does a } \\
\text { wrist motion }\end{array}$ & $\begin{array}{c}\checkmark \\
\text { User selects key and } \\
\text { points to lock }\end{array}$ \\
\hline
\end{tabular}




\begin{tabular}{|c|c|c|c|c|c|}
\hline $\begin{array}{l}\text { typing on a } \\
\text { keyboard }\end{array}$ & typing on a keyboard & $\begin{array}{l}\text { pushing buttons with all of } \\
\text { my fingers }\end{array}$ & $\begin{array}{l}\text { talking to the } \\
\text { computer instead - } \\
\text { voice recogintion? }\end{array}$ & & $\begin{array}{c}\checkmark \\
\text { Selecting keys }\end{array}$ \\
\hline USB stick & $\begin{array}{l}\text { interacting with object } \\
\text { because I want to } \\
\text { manipulate files (i.e.: save, } \\
\text { move, copy) } \\
\text { accomplishing: successfully } \\
\text { transferred (for backup } \\
\text { purposes) and saved a file. }\end{array}$ & $\begin{array}{l}\text { i removed the cap and } \\
\text { plugged it into the USB slot } \\
\text { of my computer, it } \\
\text { automatically opened a } \\
\text { window and I dragged and } \\
\text { dropped a file from my hard } \\
\text { drive into the USB stick. }\end{array}$ & & $\begin{array}{c}\checkmark \\
\text { Remove } \\
\text { cap/insertion, } \\
\text { mouse }\end{array}$ & $\begin{array}{c}\checkmark \\
\text { Select items using } \\
\text { mouse in on screen } \\
\text { display }\end{array}$ \\
\hline $\begin{array}{l}\text { my cell } \\
\text { phone }\end{array}$ & $\begin{array}{l}\text { because i need to call some } \\
\text { one. } \\
\text { accomplishing: being } \\
\text { connected to the person } \\
\text { who I wish to speak to. }\end{array}$ & $\begin{array}{l}\text { first, i flipped my phone } \\
\text { open, then i turned my } \\
\text { phone on by pressing the } \\
\text { power button, then i entered } \\
\text { my unlock code, then } \\
\text { selected contacts, then i } \\
\text { scrolled through my contacts } \\
\text { list, then I selected the } \\
\text { person who i wanted to } \\
\text { contact and the phone } \\
\text { automatically dialed the } \\
\text { number. }\end{array}$ & & & $\begin{array}{c}\checkmark \\
\text { Select } \\
\text { numbers/menu } \\
\text { items on device }\end{array}$ \\
\hline $\begin{array}{r}\text { Radio } \\
\text { Controls }\end{array}$ & Tuning and volume & $\begin{array}{l}\text { button presses to change } \\
\text { state } \\
\text { turning dial for volume } \\
\text { change } \\
\text { button press for on/off }\end{array}$ & $\begin{array}{l}\text { Single state } \\
\text { buttons } \\
\text { Range Slider for } \\
\text { volume }\end{array}$ & & $\begin{array}{c}\checkmark \\
\text { Select } \\
\text { numbers/menu } \\
\text { items on device }\end{array}$ \\
\hline$T V$ & Buttons in all cases & & & & $\begin{array}{c}\checkmark \\
\text { Select } \\
\text { numbers/menu }\end{array}$ \\
\hline
\end{tabular}




\begin{tabular}{|c|c|c|c|c|c|}
\hline & & & & & $\begin{array}{c}\text { items on } \\
\text { device/remote }\end{array}$ \\
\hline $\begin{array}{l}\text { Washing } \\
\text { machine }\end{array}$ & Wash clothes & $\begin{array}{l}\text { Select washing mode on dial } \\
\text { Start load }\end{array}$ & $\begin{array}{l}\text { Segmented slider, } \\
\text { labeled with all } \\
\text { modes } \\
\text { Buttons to start } \\
\text { the load, set } \\
\text { modifiers }\end{array}$ & & $\begin{array}{c}\checkmark \\
\text { Select } \\
\text { numbers/menu } \\
\text { items on device }\end{array}$ \\
\hline $\begin{array}{r}\text { Dimmer } \\
\text { switch }\end{array}$ & Operate lights & $\begin{array}{l}\text { On/Off switch } \\
\text { Graduated slider to control } \\
\text { light level }\end{array}$ & Button, and slider & & $\begin{array}{c}\checkmark \\
\text { Select on/off or } \\
\text { slider level }\end{array}$ \\
\hline highlighter & $\begin{array}{l}\text { to highlight text that I think } \\
\text { is important and that I would } \\
\text { want to notice right away } \\
\text { the next time i look at this } \\
\text { paper. } \\
\text { accomplishing: make text } \\
\text { stand out more. }\end{array}$ & $\begin{array}{l}\text { i removed the cap with one } \\
\text { hand and then with the } \\
\text { highlighter in my other hand, } \\
\text { i drew a thick line across a } \\
\text { sentence to highlight it. }\end{array}$ & & $\begin{array}{c}\checkmark \\
\text { Swipe motion }\end{array}$ & $\begin{array}{c}\checkmark \\
\text { Select the text } \\
\text { location }\end{array}$ \\
\hline water bottle & $\begin{array}{l}\text { to drink water. } \\
\text { accomplishing: pour water } \\
\text { into mouth to satisfy thirst. }\end{array}$ & $\begin{array}{l}\text { i picked it up, turned the cap } \\
\text { to remove it, then I took a sip } \\
\text { of water from it. Result was } \\
\text { that the water would transfer } \\
\text { from the bottle to mouth. }\end{array}$ & & $\begin{array}{c}\checkmark \\
\text { Removed } \\
\text { cap/bottle } \\
\text { motion }\end{array}$ & \\
\hline
\end{tabular}




\section{Appendix B: PowerPoint Controller Show XML}

Provided here for quick reference is a sample XML format DMIP API layout file. The client representation of which is viewable in Figure 14: Windows Forms Client and Figure 16:

\section{PowerPoint Controller from Android.}

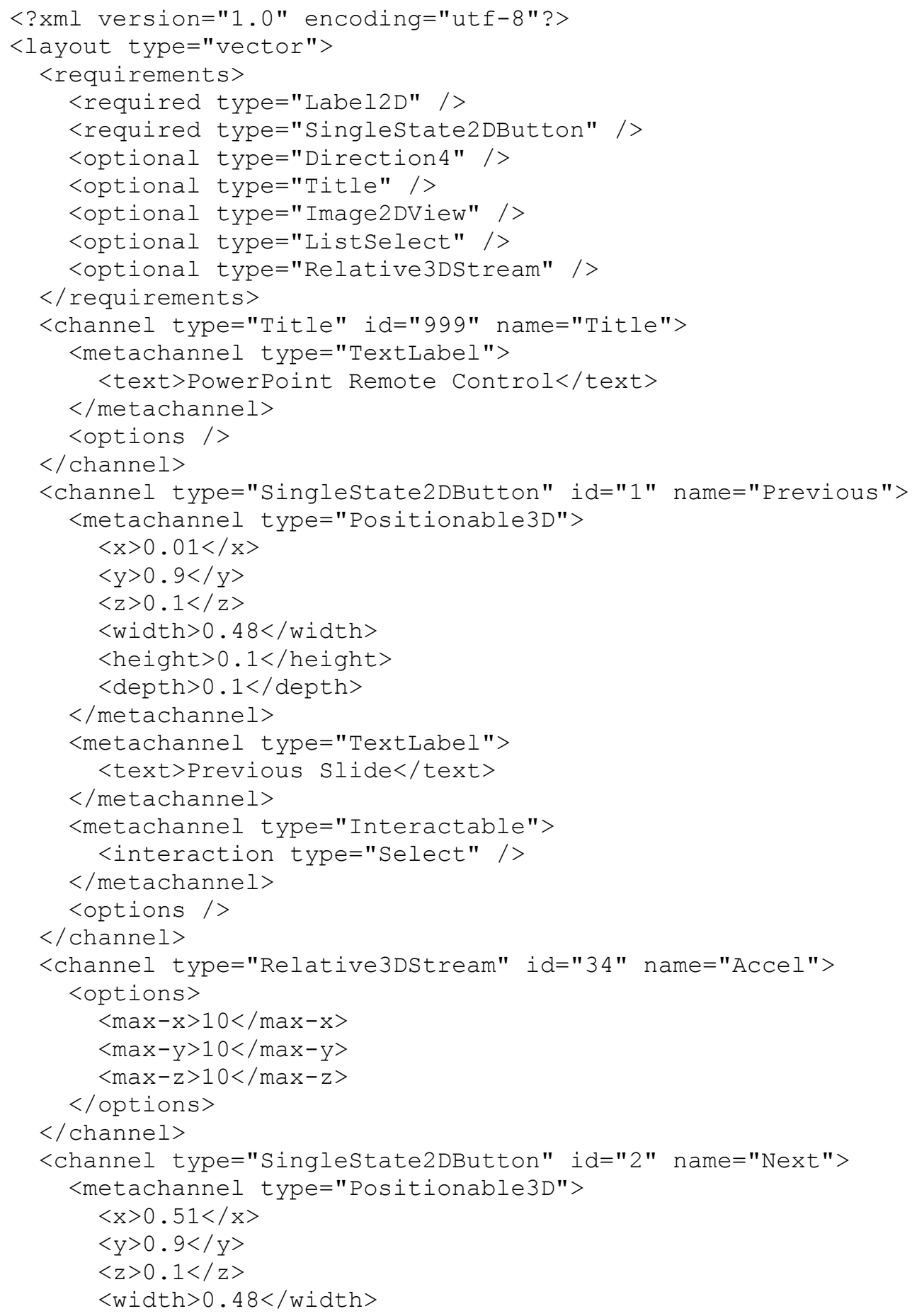




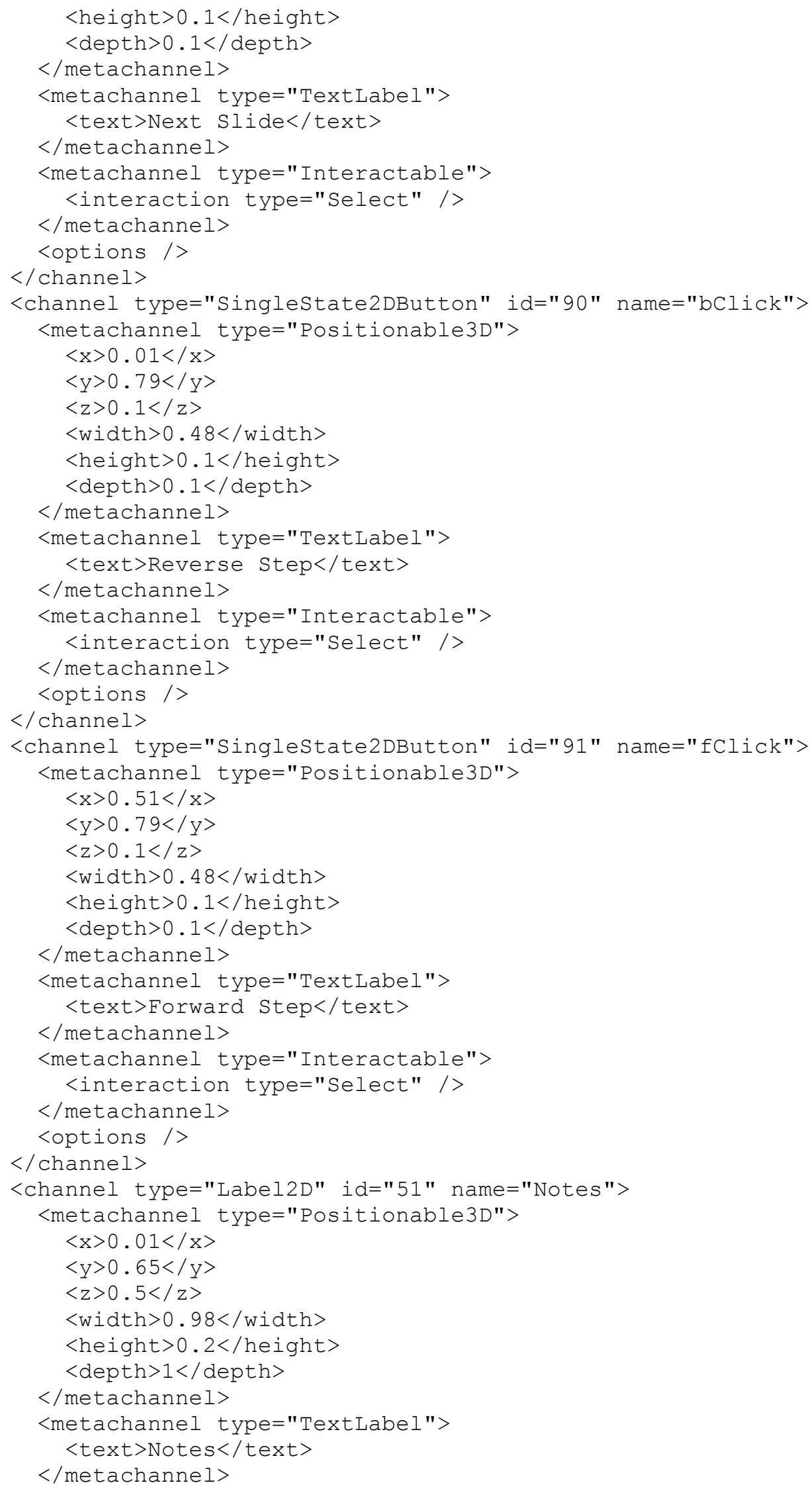




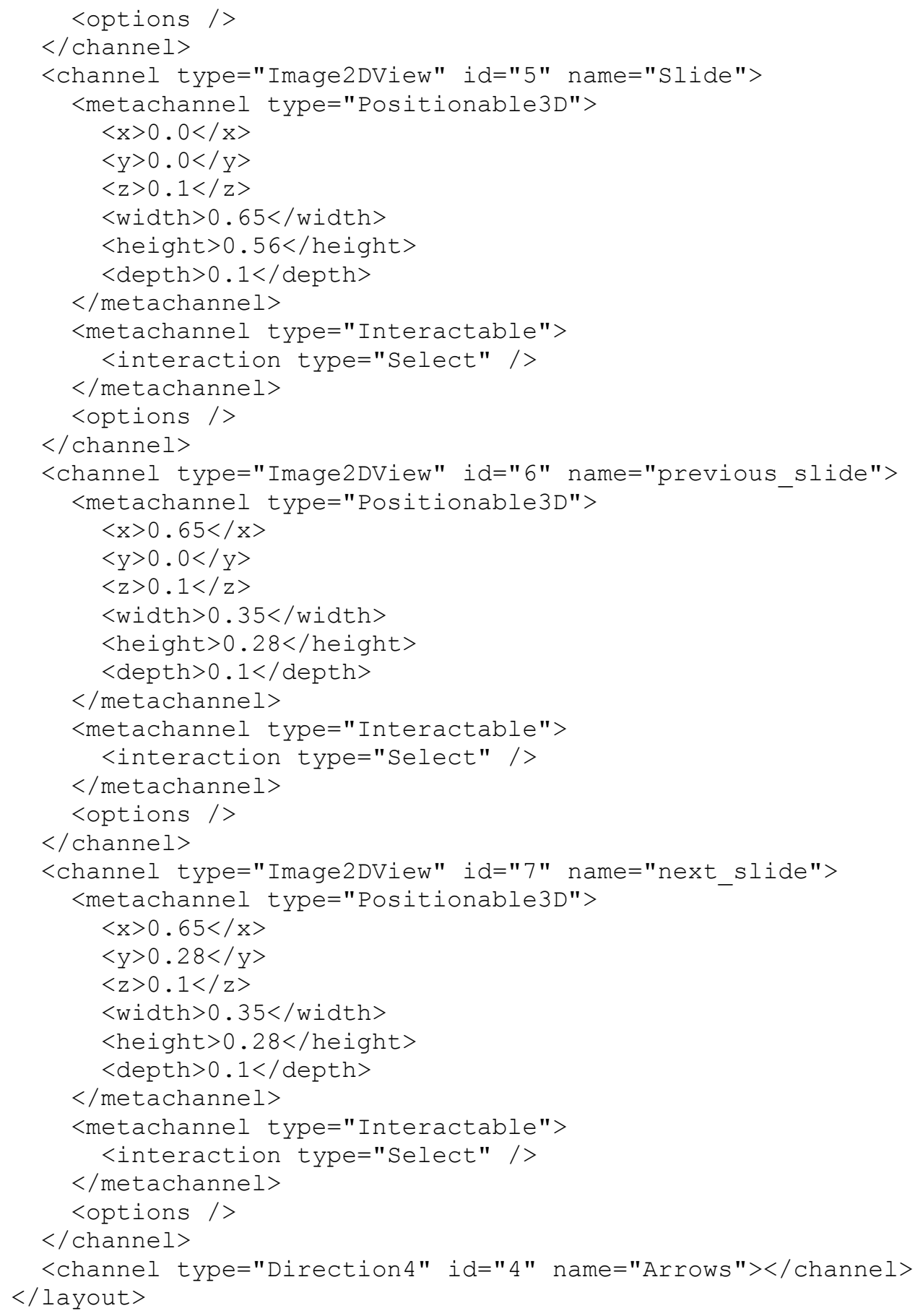

